\title{
Leptogenesis from first principles in the resonant regime
}

\author{
Mathias Garny $^{a *}$, Alexander Kartavtsev ${ }^{b}$, Andreas Hohenegger ${ }^{c}$ \\ a Deutsches Elektronen-Synchrotron DESY, \\ Notkestrasse 85, 22603 Hamburg, Germany \\ b Max-Planck-Institut für Kernphysik, \\ Saupfercheckweg 1, 69117 Heidelberg, Germany \\ c École Polytechnique Fédérale de Lausanne \\ CH-1015 Lausanne, Switzerland
}

July 11, 2013

\begin{abstract}
The lepton asymmetry generated by the out-of-equilibrium decays of heavy Majorana neutrinos with a quasi-degenerate mass spectrum is resonantly enhanced. In this work, we study this scenario within a first-principle approach. The quantum field theoretical treatment is applicable for mass splittings of the order of the width of the Majorana neutrinos, for which the enhancement is maximally large. The non-equilibrium evolution of the mixing Majorana neutrino fields is described by a formal analytical solution of the Kadanoff-Baym equations, that is obtained by neglecting the back-reaction. Based on this solution, we derive approximate analytical expressions for the generated asymmetry and compare them to the Boltzmann result. We find that the resonant enhancement obtained from the Kadanoff-Baym approach is smaller compared to the Boltzmann approach, due to additional contributions that describe coherent transitions between the Majorana neutrino species. We also discuss corrections to the masses and widths of the degenerate pair of Majorana neutrinos that are relevant for very small mass splitting, and compare the approximate analytical result for the lepton asymmetry with numerical results.
\end{abstract}

*mathias.garny@desy.de; +49-40-8998-3519 


\section{Introduction}

The almost complete absence of antimatter on Earth, in the solar system and in hadronic cosmic rays suggests that the universe is baryonically asymmetric. This conclusion is confirmed by experimental data on the abundances of the light elements [1] and precise measurements of the cosmic microwave background spectrum $[2,3]$. The baryon asymmetry of the universe can be generated dynamically provided the three Sakharov conditions [4] are fulfilled in the early universe: violation of baryon (or baryon minus lepton) number; violation of $C$ and $C P$; and deviation from thermal equilibrium. Although the Sakharov conditions are fulfilled in the Standard Model of particle physics (SM), the smallness of the $C P$-violation in the quark sector and the fact that, given the current bounds on the Higgs mass, the electroweak transition is not of first order, do not allow for the generation of an asymmetry comparable to the observed one. Therefore, the question about the origin of this asymmetry represents a major puzzle of modern physics.

From the theoretical point of view a very attractive explanation of the observed baryon asymmetry of the Universe is provided by the baryogenesis via leptogenesis scenario [5]. In this scenario the Standard Model is supplemented by at least two right-handed Majorana neutrinos $N_{i}$ which couple to leptons and the Higgs:

$$
\mathcal{L}=\mathcal{L}_{S M}+\frac{1}{2} \bar{N}_{i}\left(i \not \partial-M_{i}\right) N_{i}-h_{\alpha i} \overline{\ell_{\alpha}} \tilde{\phi} P_{R} N_{i}-h_{i \alpha}^{\dagger} \bar{N}_{i} \tilde{\phi}^{\dagger} P_{L} \ell_{\alpha},
$$

where $\phi$ and $\ell$ are the Higgs and lepton doublets and $\tilde{\phi} \equiv i \sigma_{2} \phi^{*}$. We work in the mass eigenbasis of the Majorana neutrinos, $M=\operatorname{diag}\left(M_{i}\right)$, which are $C P$ eigenstates with parities $\eta_{i}= \pm 1, N_{i}^{c}=\eta_{i} N_{i}$. The Majorana condition implies in particular that $\bar{N}$ and $N$ are not independent but related by $\bar{N}_{i}=\eta_{i} N_{i}^{T} C$ where $C=i \gamma_{2} \gamma_{0}$ is the charge-conjugation matrix. This minimal extension of the SM can explain naturally the observed small mass scale of the active neutrinos via the famous see-saw mechanism. Furthermore, $C P$-violating decays are responsible for the generation of a lepton asymmetry, i.e. for leptogenesis, which is converted into the observed baryon asymmetry by the sphaleron processes [6].

The $C P$-violating parameter receives contributions from the vertex [5] and self-energy $[7,8,9,10]$ diagrams. If the masses of the heavy neutrinos are strongly hierarchical, then the two contributions are comparable. On the other hand, if the mass spectrum is quasidegenerate then the self-energy contribution is resonantly enhanced and becomes considerably larger than the one from the vertex diagram. It has been argued that the resonant enhancement of the $C P$ violating parameter allows one to bring the scale of leptogenesis down to $\sim 1 \mathrm{TeV}$ $[10,11]$. The lightness of the right-handed neutrinos can in principle induce a detectable non-unitarity of the mixing matrix of active neutrinos $[12,13]$ which is very interesting from the theoretical and experimental point of view. In addition, provided the right-handed neutrinos are lighter than about $10^{7} \mathrm{GeV}$, their contribution to the radiative corrections of the Higgs mass are of the order of or below the TeV scale [14]. Therefore one may argue that the existence of right-handed neutrinos below $10^{7} \mathrm{GeV}$ does not enforce the gauge hierarchy problem, even in the non-supersymmetric case.

The self-energy contribution to the $C P$-violating parameter has been extensively investigated in the literature. One of the first estimates was made in 
[15] using the effective Hamiltonian approach known from kaon physics. For a quasidegenerate mass spectrum of the heavy neutrinos, $M_{2} \simeq M_{1}$, the obtained result for the $C P$-violating parameters can be represented in the form:

$$
\epsilon_{i}=\frac{\operatorname{Im}\left[\left(h^{\dagger} h\right)_{i j}^{2}\right]}{8 \pi\left(h^{\dagger} h\right)_{i i}} R, \quad R \equiv \frac{M_{i} M_{j}\left(M_{j}^{2}-M_{i}^{2}\right)}{\left(M_{i}^{2}-M_{j}^{2}\right)^{2}+A^{2}},
$$

where $i, j=1,2$ are flavor indices, and the resonant enhancement is described by the function $R$. For $A=0$, the function $R$ would diverge in the degenerate limit. Therefore $A$ can be considered as a "regulator", that arises from the fact that the Majorana neutrinos are not strictly on-shell because of their finite lifetime. In Ref. [15] it was found

$$
A=\frac{1}{32 \pi} \operatorname{Re}\left(h^{\dagger} h\right)_{12}\left(M_{1}+M_{2}\right)^{2} .
$$

The regulator is relevant for determining the maximal possible resonant enhancement, which occurs for $M_{2}^{2}-M_{1}^{2}= \pm A$, and is given by

$$
R_{\max }=\frac{M_{1} M_{2}}{2|A|}
$$

Note that (2) is proportional to the product $M_{1} M_{2}\left(M_{2}^{2}-M_{1}^{2}\right) \operatorname{Im}\left(h^{\dagger} h\right)_{12}^{2}$ which, similarly to the Jarlskog determinant, is an invariant (under field rotations) quantity characterizing $C P$-violation in the leptonic sector. In particular, if the heavy neutrino masses are equal, i.e. if $M_{1}^{2}=M_{2}^{2}$, then the $C P$-violating phases in the Yukawa couplings can be rotated away and the $C P$-violating parameter vanishes. Extending the formalism developed in [16] the authors of [17] studied the effects of particle mixing for scalar fields with arbitrary mass splitting and also found a considerable enhancement of the asymmetries when the masses of the mixed states are comparable. For decreasing mass splitting the maximal enhancement is achieved for $M_{2}^{2}-M_{1}^{2} \simeq \Gamma_{11}^{2}+\Gamma_{22}^{2}$, where $\Gamma_{i j}$ denotes the absorptive part of the one-loop self-energy diagram. This computation was adapted to the mixing of neutrinos in [18].

An approach based on an expansion of full resummed propagators around their poles was developed and applied to the analysis of leptogenesis in the SM in $[9,19,20,10]$. A related approach based on the diagonalization of the resummed propagator was developed in Ref. [21]. The starting point of both approaches is the solution of the Schwinger-Dyson equation for the full propagator of the mixing species in vacuum. The latter was used in Ref. [9] to extract the CP-violating decay rate based on a generalized S-Matrix for unstable, mixing particles. In Ref. [21] a strategy was followed that is conceptually different, namely the resummed propagators were substituted into amplitudes of the lepton-number violating scattering processes, for which only stable particles appear in the asymptotic in and out states. The arising expressions are then interpreted in terms of effective decay widths of the Majorana neutrino species which are used to calculate the corresponding $C P$-violating parameters. The result obtained for the $C P$-violating parameter in the pole expansion method [9] has the form of Eq. (2) but with a "regulator" given by

$$
A=M_{i} \Gamma_{j}
$$


The result obtained for the $C P$-violating parameter using the diagonalization approach [21] also has the form of Eq. (2), but with

$$
A=M_{i} \Gamma_{i}-M_{j} \Gamma_{j}
$$

As has been noted in Ref. [22], the difference can be traced back to the way how the $C P$-violating decay rates are extracted from the scattering amplitudes. Furthermore, it has been argued that both the pole expansion and diagonalization approach can be reconciled, and yield a regulator of the form (6). In general there exist two potential sources that can contribute to the regulator, arising from the finite width of the two Majorana neutrino species required for a $C P$-violating contribution to the decay rate. Therefore it seems plausible that the regulator depends on the widths of both species. As is evident from Eq. (4), the latter result predicts a larger value of the $C P$-violating parameter, especially if $\Gamma_{i} \sim \Gamma_{j}$. A comparison of the various approaches to the calculation of the $C P$-violating parameter in the resonant regime can also be found in [23].

In all of the above works the $C P$-violating parameters are evaluated in vacuum and then used to calculate the asymmetry generated by the decays of the heavy neutrinos. In the hot and dense plasma thermal corrections to the decay and scattering rates can play an important role. An analysis of leptogenesis within thermal quantum field theory was performed in $[24,25,26]$. The influence of thermal corrections on the dispersion relations was studied in [27, 28, 29]. The production rate of right-handed neutrinos in a thermal plasma of SM particles has been studied in $[30,31]$ and in $[32,33]$ for temperatures that are large or small compared to their mass, respectively.

The resonant enhancement of the $C P$-violating parameter becomes maximal when the mass difference of the neutrinos is of the order of their widths. Expressed in a quantum field theoretical language, this means that the spectral functions of the neutrinos cannot be described by two distinct peaks, but rather by two overlapping resonances. In addition, this implies that it is not sufficient to describe the deviation from equilibrium in terms of semi-classical distribution functions $f_{N_{i}}(t)$ for the two neutrinos $i=1,2$. Instead, it is important to take a full two-by-two matrix into account, that can describe coherent transitions between the neutrino species in terms of cross-correlations. Within a purely quantum field theoretical treatment, a natural object to consider are the two-point functions $\left\langle\bar{N}_{i}(x) N_{j}(y)\right\rangle$, which possess a matrix structure in flavor space. Their time-evolution can be described by the so-called closed-time-path (CTP) or Schwinger-Keldysh formalism. Concretely, self-consistent equations of motion for the non-equilibrium time-evolution can be obtained by formulating the Schwinger-Dyson equation in real time on the CTP. The resulting equations are known as Kadanoff-Baym equations. One of the first steps towards the analysis of leptogenesis within the formalism of non-equilibrium QFT has been performed in [34] where the authors solved the system of KadanoffBaym equations in Minkowski space-time using a perturbative expansion. In [35] the approach to equilibrium for a scalar field which is coupled to a large thermal bath was studied. A quantum mechanical calculation of the asymmetry generation for a hierarchical mass spectrum of the heavy neutrinos using the Kadanoff-Baym formalism was presented by the same group in [36, 37]. We also refer to Refs. [38, 39] for previous works on a quantum treatment of leptogenesis. A somewhat different approach was pursued in [40, 41, 26, 42] as well 
as in $[43,44,45]$. In these works the initial system of Kadanoff-Baym equations was used to derive Boltzmann-like equations for the quasiparticles and calculate their effective in-medium properties like masses, decay widths and $C P$-violating parameters. The vertex and self-energy contributions to the $C P$-violating parameter in a toy model with two real scalar fields and one complex scalar field were analyzed in [40] and [41]. A similar analysis for leptogenesis in the Standard Model was performed in $[43,45]$. Flavor oscillations in the leptonic sector and their impact on the generation of an asymmetry were analyzed within nonequilibrium quantum field theory in [44]. Oscillations of the right-handed neutrinos play a crucial role in the scenario of leptogenesis via oscillations [46, 47, 48]. The latter is realized, in particular, in the Neutrino Minimal Standard Model $(\nu \mathrm{MSM})[49]$. The calculation of the asymmetry generated within this scenario is based on so-called density matrix equations [50]. Another method using the perturbative short-time solution of the von Neumann equation and intended to check the consistency of the density matrix equations was presented in [51].

Summarizing the above, the analysis of leptogenesis in the maximally resonant regime poses two problems, which, despite the recent progress, are not yet fully resolved. The first one is the enhancement of the $C P$-violating parameter. The existing vacuum calculations lead to rather different results. They neglect the medium effects as well as the modification of the quasiparticle spectra in the hot and dense plasma of the early Universe. It is also a priori unclear whether the very notion of quasiparticles is applicable in the maximal resonant regime. The second problem is the oscillation of the heavy neutrinos which becomes important when the mass difference is sufficiently small. This effect is completely neglected in the standard scenario of resonant thermal leptogenesis which, in the maximal resonant regime, "merges" with the scenario of leptogenesis via oscillations.

In this work we study the generation of a lepton asymmetry from first principles using an explicit solution of the Kadanoff-Baym equations for mixing Majorana neutrinos with an arbitrary mass difference. Following Refs. [36, 37], we will treat lepton and Higgs fields as a thermal bath, neglecting the backreaction. This simplification has the advantage that it is possible to obtain analytical results that can be directly compared to the Boltzmann approach.

In section 2, we introduce the framework of our computation, explain our notations, define the quantities that are necessary for a quantum-mechanical approach to leptogenesis, and derive the relevant Kadanoff-Baym equations. In section 3 we derive an equation of motion for the lepton asymmetry, and discuss the generation of an asymmetry from the interaction of the Majorana neutrinos with a thermal bath. An analytical solution for the Kadanoff-Baym equations and the time development of the lepton asymmetry is derived in section 4 using a Breit-Wigner approximation. In section 5 we present our results obtained in the Kadanoff-Baym approach and compare it to the corresponding Boltzmann approach. In particular, we discuss the differences that arise with respect to the resonant enhancement of the lepton asymmetry. We also compare the solutions of the Kadanoff-Baym equations for various approximations. Finally, we conclude in section 6 . Details of the derivation of the analytical solution can be found in the Appendix. 


\section{Closed-time-path formalism}

Leptogenesis is a non-equilibrium process. Within quantum field theory, such processes can be conveniently described using the closed-time-path or SchwingerKeldysh formalism. In particular, we are interested in the non-equilibrium timeevolution of the expectation value of physical observables, like the baryon or lepton asymmetry. Consider for example the lepton current operator,

$$
J_{L}^{\mu}(x)=\sum_{\alpha} \bar{\ell}_{\alpha}(x) \gamma^{\mu} \ell_{\alpha}(x) .
$$

We are interested in the time-evolution of its expectation value with respect to the physical state of the system,

$$
j_{L}^{\mu}(x)=\left\langle J_{L}^{\mu}(x)\right\rangle=\left\langle\sum_{\alpha} \bar{\ell}_{\alpha}(x) \gamma^{\mu} \ell_{\alpha}(x)\right\rangle .
$$

The lepton asymmetry per volume $V$ is then given by

$$
n_{L}(t)=\frac{1}{V} \int_{V} d^{3} x j_{L}^{0}(t, \boldsymbol{x}) .
$$

It is the main purpose of this work to compute the time-evolution of the lepton asymmetry that is generated by the out of equilibrium decays of quasidegenerate Majorana neutrino species within the closed time path formalism. In the following, we will introduce the quantities and equations that are necessary for this computation.

\section{$2.1 \quad$ Propagators}

Within quantum field theory an out-of-equilibrium system can be characterized completely by its full set of $N$-point correlation functions. Since we are interested in a temperature regime for which the electroweak symmetry is unbroken, the lowest-order correlations are the following two-point functions

$$
\begin{aligned}
\Delta_{\phi_{a b}}(x, y) & =\left\langle T_{\mathcal{C}} \phi^{a}(x) \phi^{* b}(y)\right\rangle, \\
S_{\ell_{a b}^{\alpha \beta}}^{\alpha \beta}(x, y) & =\left\langle T_{\mathcal{C}} \ell_{\alpha}^{a}(x) \bar{\ell}_{\beta}^{b}(y)\right\rangle, \\
S^{i j}(x, y) & =\left\langle T_{\mathcal{C}} N_{i}(x) \bar{N}_{j}(y)\right\rangle .
\end{aligned}
$$

Here $a, b=1,2$ are $S U(2)$ indices, and $\alpha, \beta=1,2,3$ as well as $i, j=1,2,3$ are flavor indices of the leptons and Majorana neutrinos respectively. We use matrix notation for the Lorentz-spinor indices. $T_{\mathcal{C}}$ denotes time-ordering with respect to the closed time contour, and features the usual minus sign when interchanging fermionic operators. In an $S U(2)$-symmetric state, which we consider here, one can write

$$
\begin{aligned}
\Delta_{\phi_{a b}}(x, y) & =\delta_{a b} \Delta_{\phi}(x, y), \\
S_{\ell_{a b}}^{\alpha \beta}(x, y) & =\delta_{a b} S_{\ell}^{\alpha \beta}(x, y) .
\end{aligned}
$$

Note that the lepton and Majorana neutrino propagators are matrices in flavor space, i.e. they encode cross-correlations which are important for coherence effects. Throughout this work we assume that the third Majorana neutrino is very 
heavy, and therefore consider the two-by-two matrix in flavor space corresponding to $N_{1,2}$. We will frequently use matrix notation for the flavor indices. If the two-point functions are known, the lepton current can be easily calculated,

$$
j_{L}^{\mu}(x)=-\sum_{\alpha} \operatorname{tr}\left[\gamma_{\mu} S_{\ell}^{\alpha \alpha}(x, x)\right] .
$$

Thus, the main task will be to obtain solutions of the non-equilibrium evolution equations for the two-point functions, so-called Kadanoff-Baym equations, and to extract the relevant processes which describe the generation of the lepton asymmetry.

By denoting the time arguments on the chronological branch of the closedtime contour by $x^{+}$and the one on the anti-chronological branch by $x^{-}$, one can obtain four propagators with usual real time arguments,

$$
\begin{array}{ll}
D_{++}(x, y)=D\left(x^{+}, y^{+}\right), & D_{+-}(x, y)=D\left(x^{+}, y^{-}\right), \\
D_{-+}(x, y)=D\left(x^{-}, y^{+}\right), & D_{--}(x, y)=D\left(x^{-}, y^{-}\right),
\end{array}
$$

where $D(x, y)$ stands for any two-point function. The four functions are often combined into a two-by-two matrix structure (in addition to the flavor and Dirac indices). However, it is easy to see that only two of them are independent. In this work, we prefer to use the more compact notation of [52], and decompose the two-point function into spectral function $D_{\rho}$ and statistical propagator $D_{F}$,

$$
D(x, y)=D_{F}(x, y)-\frac{i}{2} \operatorname{sgn}_{\mathcal{C}}\left(x^{0}-y^{0}\right) D_{\rho}(x, y) .
$$

The signum function is either +1 or -1 depending on whether $x^{0}$ or $y^{0}$ occur 'later' on the closed time contour $\mathcal{C}$. From this parameterization it is evident that in fact only two independent two-point functions exist. All other twopoint functions can be expressed as linear combinations. For example, one can introduce the Wightman functions $D_{\gtrless}(x, y)=D_{F}(x, y) \mp \frac{i}{2} D_{\rho}(x, y)$. In terms of the matrix notation, these would correspond to $D_{>}=D_{-+}$and $D_{<}=D_{+-}$. Finally, it is useful to introduce retarded and advanced functions,

$$
\begin{aligned}
& D_{R}(x, y)=\Theta\left(x^{0}-y^{0}\right) D_{\rho}(x, y), \\
& D_{A}(x, y)=-\Theta\left(y^{0}-x^{0}\right) D_{\rho}(x, y) .
\end{aligned}
$$

A useful relation for the Majorana neutrino propagator, which results from the relation $N_{i}^{c}=\eta_{i} N_{i}$, is

$$
S^{i j}(x, y)=\eta_{i} \eta_{j} C S^{j i}(y, x)^{T} C^{-1},
$$

implying that

$$
\begin{aligned}
S_{F, R / A}^{i j}(x, y) & =\eta_{i} \eta_{j} C S_{F, A / R}^{j i}(y, x)^{T} C^{-1}, \\
S_{\rho}^{i j}(x, y) & =-\eta_{i} \eta_{j} C S_{\rho}^{j i}(y, x)^{T} C^{-1} .
\end{aligned}
$$

\section{$C P$-conjugate propagators}

For leptogenesis, it is useful to consider separately $C P$-odd and $C P$-even effects. In particular, the generation of the lepton asymmetry is a $C P$-odd effect. In 
order to discriminate between $C P$-odd and $C P$-even, one can perform a $C P$ transformation of the system, and determine the transformation properties of the two-point functions.

The Higgs field transforms like $\phi(x) \rightarrow \phi^{*}(\bar{x})$ under $C P$, where $\bar{x} \equiv\left(x^{0},-\mathbf{x}\right)$. Thus the propagator gets transformed in the following way:

$$
\Delta_{\phi_{a b}}(x, y)=\left\langle T_{\mathcal{C}} \phi_{a}(x) \phi_{b}^{*}(y)\right\rangle \rightarrow\left\langle T_{\mathcal{C}} \phi_{a}^{*}(\bar{x}) \phi_{b}(\bar{y})\right\rangle=\Delta_{\phi_{b a}}(\bar{y}, \bar{x}) .
$$

Motivated by this observation, we define the $C P$-conjugate propagator as

$$
\bar{\Delta}_{\phi_{a b}}(x, y) \equiv \Delta_{\phi_{b a}}(\bar{y}, \bar{x}) .
$$

From this, one can deduce the various components introduced above,

$$
\begin{aligned}
\bar{\Delta}_{\phi_{a b F, R / A}}(x, y) & =\Delta_{\phi_{b a} F, A / R}(\bar{y}, \bar{x}), \\
\bar{\Delta}_{\phi_{a b \rho} \rho}(x, y) & =-\Delta_{\phi_{b a} \rho}(\bar{y}, \bar{x}) .
\end{aligned}
$$

It is important to note that for a system in a CP-symmetric state the propagator and the $C P$-conjugate propagator are equal, $\bar{\Delta}_{\phi_{a b}}(x, y)=\Delta_{\phi_{a b}}(x, y)$.

The lepton field transforms like $\ell(x) \rightarrow C P \bar{\ell}^{T}(\bar{x})$ and $\bar{\ell}(x) \rightarrow \ell^{T}(\bar{x}) P C=$ $-\ell^{T}(\bar{x})(C P)^{-1}$, where $P=\gamma_{0}$. Accordingly, the propagator gets transformed like

$$
S_{\ell \mu \nu a b}^{\alpha \beta}(x, y) \rightarrow(C P)_{\mu \lambda} S_{\ell_{\sigma \lambda b a}^{\beta \alpha}}^{\beta \alpha}(\bar{y}, \bar{x})\left[(C P)^{-1}\right]_{\sigma \nu},
$$

where we have displayed Dirac indices for clarity. The $C P$-conjugate propagator is then given by

$$
\bar{S}_{\ell a b}^{\alpha \beta}(x, y) \equiv C P S_{\ell b a}^{\beta \alpha}(\bar{y}, \bar{x})^{T}(C P)^{-1} .
$$

Here we have again used a matrix-notation for the Lorentz-spinor indices, and the transpose on the right-hand side refers to these indices. For the components, one finds similarly as above

$$
\begin{aligned}
\bar{S}_{\ell_{a b \gtrless, F, R / A}^{\alpha \beta}(x, y)}^{\alpha \beta} & =C P S_{\ell_{b a \lessgtr, F, A / R}^{\beta \alpha}}^{\beta \alpha}(\bar{y}, \bar{x})^{T}(C P)^{-1}, \\
\bar{S}_{\ell a b \rho}^{\alpha \beta}(x, y) & =-C P S_{\ell b a \rho}^{\beta \alpha}(\bar{y}, \bar{x})^{T}(C P)^{-1} .
\end{aligned}
$$

For a system in a $C P$-symmetric state, one has $\bar{S}_{\ell a b}^{\alpha \beta}(x, y)=S_{\ell_{a b}}^{\alpha \beta}(x, y)$.

The $C P$-conjugate propagator of the Majorana neutrinos is given by

$$
\bar{S}^{i j}(x, y)=C P S^{j i}(\bar{y}, \bar{x})^{T}(C P)^{-1} .
$$

For the components, the relations are also analogous to the leptons.

\subsection{Kadanoff-Baym equations}

The non-equilibrium equation of motion for the two-point functions can be derived by a variational principle from the so-called two-particle-irreducible (2PI) effective action $\Gamma\left[\Delta_{\phi}, S_{\ell}, S\right]$. Technically, it is a Legendre transform with respect to the generating functional in the presence of bi-local sources [53]. All the information about the quantum system is encoded in the effective action. 


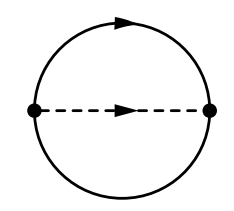

Figure 1: Two-loop diagram contributing to the 2PI effective action.
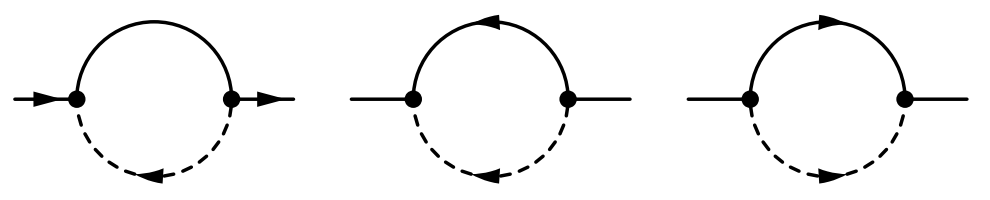

Figure 2: One-loop diagrams contributing to the lepton (left) and Majorana neutrino (middle and right) self-energies.

The equation of motion for the two-point functions follows from the stationarity conditions,

$$
\frac{\delta \Gamma}{\delta \Delta_{\phi}}=0, \quad \frac{\delta \Gamma}{\delta S_{\ell}}=0, \quad \frac{\delta \Gamma}{\delta S}=0 .
$$

In general, the $2 \mathrm{PI}$ effective action can be parameterized in the form

$$
\Gamma\left[\Delta_{\phi}, S_{\ell}, S\right]=\mathcal{S}+\Gamma^{1 L}\left[\Delta_{\phi}, S_{\ell}, S\right]+\Gamma_{2}\left[\Delta_{\phi}, S_{\ell}, S\right]
$$

where the classical action $\mathcal{S}=\int_{\mathcal{C}} d^{4} x \mathcal{L}$ is the tree-level contribution, $\Gamma^{1 L}$ the one-loop contribution, and $\Gamma_{2}$ contains diagrams with at least two loops. More precisely, $\Gamma_{2}\left[\Delta_{\phi}, S_{\ell}, S\right]$ is equal to the sum of all $2 \mathrm{PI}$ ('skeleton') Feynman diagrams which have no external legs. Furthermore, the internal lines represent the full propagators $\Delta_{\phi}, S_{\ell}$, and $S$. The two-loop contributions are shown in Fig. 1.

The resulting equations have the form of Schwinger-Dyson equations, evaluated on the closed-time path. For the leptons and the Majorana neutrinos, they read (suppressing flavor, $\mathrm{SU}(2)$ and Lorentz-spinor indices for the moment)

$$
\begin{array}{ccc}
\frac{\delta \Gamma}{\delta S_{\ell}}=0 \quad & \Leftrightarrow \quad S_{\ell}^{-1}(x, y)=S_{\ell_{0}^{-1}}(x, y)-\Sigma_{\ell}(x, y), \\
\frac{\delta \Gamma}{\delta S}=0 \quad & \Leftrightarrow \quad S^{-1}(x, y)=S_{0}^{-1}(x, y)-\Sigma_{N}(x, y) .
\end{array}
$$

Here $S_{\ell}^{-1}(x, y)$ is the inverse of the full lepton two-point function (i.e. the inverse of the full propagator), $S_{\ell_{0}}^{-1}(x, y)$ is the inverse of the free lepton propagator $^{1}$,

$$
i S_{\ell_{0}}^{-1}(x, y)=i \not \partial_{x} \delta_{a b} \delta^{\alpha \beta} \delta_{\mathcal{C}}(x-y) P_{L},
$$

${ }^{1}$ Strictly speaking, in Dirac notation the inverse exists only when restricting the spinors to the left-handed sub-space. Nevertheless it is possible to work in Dirac notation when properly inserting left-handed projectors $P_{L}$, so that effectively only the left-handed parts contribute. 
and $\Sigma_{\ell}(x, y)$ is the self-energy given by the functional derivative of the effective action $\Gamma_{2}\left[\Delta_{\phi}, S_{\ell}, S\right]$,

$$
\Sigma_{\ell \mu \nu a b}^{\alpha \beta}(x, y)=\frac{i \delta \Gamma_{2}}{\delta S_{\ell_{\nu \mu b a}^{\beta \alpha}}^{\beta \alpha}(y, x)} .
$$

Graphically, this corresponds to opening one lepton line of the diagrams contributing to $\Gamma_{2}$. Thus, for example the two-loop contribution to $\Gamma_{2}$ yields the one-loop self-energy. The one-loop contribution to the lepton self-energy is shown in Fig. 2. We stress again that the internal lines of the diagrams represent the full propagators. This means that the equation of motion is a self-consistent Schwinger-Dyson equation.

Similarly, the free inverse propagator for the Majorana neutrinos reads

$$
i S_{0}^{-1}(x, y)=\left(i \not \partial_{x}-M_{i}\right) \delta^{i j} \delta_{\mathcal{C}}(x-y) .
$$

Later on, it will be necessary to cancel divergent contributions to the self-energy of the Majorana neutrinos. Therefore, we introduce suitable mass and field counterterms. Their most general form can be obtained by applying a field rescaling $N_{i} \rightarrow\left(Z_{i j} P_{L}+\eta_{i} Z_{i j}^{*} \eta_{j} P_{R}\right) N_{j}$ compatible with the Majorana conditions and using the most general form of the mass matrix for the mass counterterms, $\delta M_{i j}=\eta_{i} \delta M_{j i} \eta_{j}$. The resulting counterterms are given by $[10,54]$

$$
\begin{aligned}
\delta \mathcal{L}= & \frac{1}{2} \bar{N}\left\{\frac{i}{2}\left(\delta Z^{\dagger}+\delta Z\right) P_{R} \not \partial+\frac{i}{2}\left(\eta \delta Z^{T} \eta+\eta \delta Z^{*} \eta\right) P_{L} \not \partial-\frac{1}{2}\left(\delta Z^{\dagger} M+2 \delta M^{\dagger}\right.\right. \\
& \left.\left.+M \eta \delta Z^{*} \eta\right) P_{R}-\frac{1}{2}\left(\eta \delta Z^{T} \eta M+2 \delta M+M \delta Z\right) P_{L}\right\} N .
\end{aligned}
$$

The (renormalized) self-energy has two contributions,

$$
\Sigma_{N \mu \nu}^{i j}(x, y)=\frac{i \delta \Gamma_{2}}{\delta S_{\nu \mu}^{j i}(y, x)}+\delta \Sigma_{N \mu \nu}^{i j}(x, y) .
$$

The latter arises from the counterterms (26),

$$
\begin{aligned}
\delta \Sigma_{N}{ }^{i j}(x, y) & =-i \delta \Sigma_{N}^{i j}(x) \delta_{\mathcal{C}}(x-y), \\
\delta \Sigma_{N}(x) & =-\left\{\frac{i}{2}\left(\delta Z^{\dagger}+\delta Z\right) P_{R} \not \partial_{x}+\frac{i}{2}\left(\eta \delta Z^{T} \hat{\eta}+\eta \delta Z^{*} \eta\right) P_{L} \not_{x}-\frac{1}{2}\left(\delta Z^{\dagger} M\right.\right. \\
& \left.\left.+2 \delta M^{\dagger}+M \eta \delta Z^{*} \eta\right) P_{R}-\frac{1}{2}\left(\eta \delta Z^{T} \eta M+2 \delta M+M \delta Z\right) P_{L}\right\} .
\end{aligned}
$$

The Majorana self-energy inherits the property

$$
\Sigma_{N}^{i j}(x, y)=\eta_{i} \eta_{j} C \Sigma_{N}^{j i}(y, x)^{T} C^{-1},
$$

of the Majorana propagator. Note that also the counterterm-part fulfills this relation.

The equation of motion can be rewritten in a more explicit form by convoluting it with the full propagator, and inserting the decomposition into statistical 
and spectral components. Then one obtains a system of two coupled integrodifferential equations, so-called Kadanoff-Baym equations,

$$
\begin{aligned}
i \not \partial_{x} S_{\ell_{F}}^{\alpha \beta}(x, y) & =\int_{0}^{x^{0}} d^{4} z \Sigma_{\ell \rho}^{\alpha \gamma}(x, z) S_{\ell_{F}}^{\gamma \beta}(z, y) \\
& -\int_{0}^{y^{0}} d^{4} z \Sigma_{\ell F}^{\alpha \gamma}(x, z) S_{\ell_{\rho}^{\gamma \beta}}^{\gamma \beta}(z, y), \\
i \not_{x} S_{\ell \rho}^{\alpha \beta}(x, y) & =\int_{y^{0}}^{x^{0}} d^{4} z \Sigma_{\ell \rho}^{\alpha \gamma}(x, z) S_{\ell \rho}^{\gamma \beta}(z, y) .
\end{aligned}
$$

The corresponding Kadanoff-Baym equations for the Majorana neutrinos read (in matrix notation for the flavor indices)

$$
\begin{aligned}
\left(i \not \partial_{x}-M-\delta \Sigma_{N}(x)\right) S_{F}(x, y) & =\int_{0}^{x^{0}} d^{4} z \Sigma_{N \rho}(x, z) S_{F}(z, y) \\
& -\int_{0}^{y^{0}} d^{4} z \Sigma_{N F}(x, z) S_{\rho}(z, y), \\
\left(i \not \partial_{x}-M-\delta \Sigma_{N}(x)\right) S_{\rho}(x, y) & =\int_{y^{0}}^{x^{0}} d^{4} z \Sigma_{N \rho}(x, z) S_{\rho}(z, y) .
\end{aligned}
$$

These equations contain 'memory integrals' on the right-hand side, which integrate over the history of the system. Thus, the change of the two-point function at a given instant of time depends on the entire history of the system, i.e. the equations are non-Markovian. Furthermore, the equations explicitly depend on the two time-arguments $x^{0}$ and $y^{0}$ characterizing the two-point function. For a system in equilibrium, they would only depend on the difference $s^{0}=x^{0}-y^{0}$. Out of equilibrium, the system also depends on the average time $X^{0}=\left(x^{0}+y^{0}\right) / 2$. For this reason, the equations in the form given above are sometimes referred to as two-time Kadanoff-Baym equations. We stress that these equations are valid also far from equilibrium. For systems which are close to equilibrium, one expects that the two-point functions depend only weakly on $X^{0}$. In this case, it is customary to perform a gradient expansion, which yields Markovian quantum kinetic equations. In addition, one may perform a Fourier transformation with respect to $s^{0}$ (Wigner transformation). Then, a very common further simplification consists of making a particular ansatz for the frequency-dependence of the two-point functions, including a Breit-Wigner ansatz or a quasi-particle ansatz. The latter is known to lead to Boltzmann-type equations.

On the other hand, it is also possible to follow a different strategy, namely to retain the non-Markovian, two-time structure of the equations. If, in addition, the self-energies are computed using the complete propagators, the resulting equations are non-linear integro-differential equations. Equations of this type have been solved numerically with increasing complexity over the past years, and it has been shown that they can describe the process of kinetic and chemical quantum thermalization for systems that are initially far from equilibrium, see e.g. $[55,56,57,58,59,60,61]$. A significant simplification can be achieved when treating the self-energies as time-translation invariant functions, which reduces the Kadanoff-Baym equations to linear integro-differential equations. 
Physically, this can occur when the quantum field that is out of equilibrium is weakly interacting with a thermal bath, and the interaction can be described by self-energies that solely depend on propagators associated to particle species belonging to the thermal bath. The approximation of time-translation invariant self-energies then corresponds to neglecting the backreaction of the nonequilibrium quantum field on the thermal bath (more precisely, to neglect the possible deviation from equilibrium of the self-energies that is caused by the backreaction). For that case, approximate analytical solutions of the KadanoffBaym equations within the two-time representation have been derived in [35]. Moreover, these solutions turn out to be well-suited for describing the evolution of the Majorana neutrino propagator, and have been used to study leptogenesis in the hierarchical limit [36, 37]. The reason is twofold: First, the corresponding self-energies depend on the lepton and Higgs propagators, and the latter belong to the thermal bath of $\mathcal{O}(100) \mathrm{SM}$ particles that are kept in equilibrium by the gauge interactions. Second, the Majorana neutrino itself interacts weakly with the SM via the Yukawa couplings. In the present work, we will follow the same strategy to obtain analytical solutions of the Kadanoff-Baym equations for the resonant case.

Although the two-time Kadanoff-Baym equations shown above are valid far from equilibrium and do not require any a priori assumptions on the spectral properties, they do have a restriction which is not so commonly emphasized. Namely, the equations in the above form implicitly assume that the initial state of the system, corresponding to $x^{0}=y^{0}=0$, is a Gaussian initial state. This means that the connected part of all higher correlation functions (three-, fourpoint correlations and so on) vanish at the initial time. Physically, this means that the interactions are 'switched on' just at the time $t=0$. However, it is possible to generalize the Kadanoff-Baym equations such that they can also describe systems with more realistic initial conditions, if necessary [62]. Below, we will in fact include higher correlations in an effective way by taking the evolution of the system before the 'initial' time into account.

\section{$2.3 \quad$ Self-energies}

The self-energies on the right-hand side of the Kadanoff-Baym equations contain all the information about the interactions of the system. As discussed above, the one-loop self-energy is obtained from the two-loop contribution to the 2PI effective action (see Fig. 1),

$$
\begin{aligned}
\Gamma_{2}^{2 L}\left[\Delta_{\phi}, S_{\ell}, S\right]= & \sum_{i j \alpha \beta} h_{i \alpha}^{\dagger} h_{\beta j} \int_{\mathcal{C}} d^{4} x \int_{\mathcal{C}} d^{4} y \operatorname{tr}\left[S^{j i}(y, x) P_{L} S_{\ell a c}^{\alpha \beta}(x, y) P_{R}\right] \\
& \times \epsilon^{b a} \epsilon^{c d} \Delta_{\phi b d}(x, y) .
\end{aligned}
$$

The one-loop lepton self-energy obtained by differentiating $\Gamma_{2}^{2 L}$ with respect to the full lepton propagator $S_{\ell}(y, x)$ reads (see the first diagram in Fig. 2),

$$
\Sigma_{\ell a b}^{\alpha \beta}(x, y)_{1 L}=h_{\alpha i} h_{j \beta}^{\dagger} P_{R} S^{i j}(x, y) P_{L} \epsilon^{c b} \epsilon^{a d} \Delta_{\phi_{c d}}(y, x),
$$

where summation over $i, j$ is implied. For a $\mathrm{SU}(2)$-symmetric state, one can insert that $\Delta_{\phi_{a b}}=\delta_{a b} \Delta_{\phi}$ and $S_{\ell_{a b}^{\alpha \beta}}^{\alpha \beta}=\delta_{a b} S_{\ell}^{\alpha \beta}$. Then the self-energy is itself 
proportional to $\delta_{a b}$, and one can write $\Sigma_{\ell_{a b}^{\alpha \beta}}^{\alpha \beta}=\delta_{a b} \Sigma_{\ell}^{\alpha \beta}$, with

$$
\Sigma_{\ell}^{\alpha \beta}(x, y)_{1 L}=-h_{\alpha i} h_{j \beta}^{\dagger} P_{R} S^{i j}(x, y) P_{L} \Delta_{\phi}(y, x) .
$$

The one-loop contribution to the self-energy of the Majorana neutrinos reads

$$
\begin{aligned}
\Sigma_{N}{ }^{i j}(x, y)_{1 L}= & h_{i \alpha}^{\dagger} h_{\beta j} P_{L} S_{\ell_{a c}^{\alpha \beta}}(x, y) P_{R} \epsilon^{b a} \epsilon^{c d} \Delta_{\phi_{b d}}(x, y) \\
& \eta_{i} \eta_{j} h_{i \alpha}^{T} h_{\beta j}^{*} P P_{L} \bar{S}_{\ell_{a c}^{\alpha \beta}}^{\alpha \beta}(\bar{x}, \bar{y}) P_{R} P \epsilon^{b a} \epsilon^{c d} \bar{\Delta}_{\phi_{b d}}(\bar{x}, \bar{y}) .
\end{aligned}
$$

Here summation over $\alpha, \beta$ is implied. After contraction of $\mathrm{SU}(2)$ indices

$$
\begin{aligned}
\Sigma_{N}{ }^{i j}(x, y)_{1 L}= & -2 h_{i \alpha}^{\dagger} h_{\beta j} P_{L} S_{\ell \phi}{ }^{\alpha \beta}(x, y) P_{R} \\
& -2 \eta_{i} \eta_{j} h_{i \alpha}^{T} h_{\beta j}^{*} P P_{L} \bar{S}_{\ell \phi}{ }^{\alpha \beta}(\bar{x}, \bar{y}) P_{R} P,
\end{aligned}
$$

where we have introduced an abbreviation for the lepton-Higgs loop

$$
\begin{aligned}
S_{\ell \phi}{ }^{\alpha \beta}(x, y) & \equiv S_{\ell}^{\alpha \beta}(x, y) \Delta_{\phi}(x, y) \\
\bar{S}_{\ell \phi}{ }^{\alpha \beta}(x, y) & \equiv C P S_{\ell \phi}{ }^{\beta \alpha}(\bar{y}, \bar{x})^{T}(C P)^{-1}=\bar{S}_{\ell}{ }^{\alpha \beta}(x, y) \bar{\Delta}_{\phi}(x, y) .
\end{aligned}
$$

Similar to the propagators, it is useful to define $C P$-conjugate self-energies,

$$
\begin{aligned}
& \bar{\Sigma}_{\ell}^{\alpha \beta}(x, y) \equiv C P \Sigma_{\ell}^{\beta \alpha}(\bar{y}, \bar{x})^{T}(C P)^{-1}, \\
& \bar{\Sigma}_{N}^{i j}(x, y) \equiv C P \Sigma_{N}^{j i}(\bar{y}, \bar{x})^{T}(C P)^{-1} .
\end{aligned}
$$

Then one finds for the $C P$-conjugated one-loop lepton self-energy,

$$
\bar{\Sigma}_{\ell}^{\alpha \beta}(x, y)_{1 L}=-h_{\alpha i}^{*} h_{j \beta}^{T} P_{R} \bar{S}^{i j}(x, y) P_{L} \bar{\Delta}_{\phi}(y, x) .
$$

and for the Majorana neutrino

$$
\begin{aligned}
\bar{\Sigma}_{N}{ }^{i j}(x, y)_{1 L}= & -2 h_{i \alpha}^{T} h_{\beta j}^{*} P_{L} \bar{S}_{\ell \phi}{ }^{\alpha \beta}(x, y) P_{R} \\
& -2 \eta_{i} \eta_{j} h_{i \alpha}^{\dagger} h_{\beta j} P P_{L} S_{\ell \phi}{ }^{\alpha \beta}(\bar{x}, \bar{y}) P_{R} P .
\end{aligned}
$$

\section{Equation of motion for the lepton asymmetry}

The most important observable for leptogenesis is the lepton asymmetry. Its time-evolution is usually computed by subtracting the lepton and anti-lepton particle densities. However, since particle number distributions are a classical concept, we have to replace them by a suitable quantum-mechanical observable. A suitable starting point is the operator

$$
J_{L \alpha \beta}^{\mu}(x)=\bar{\ell}_{\alpha}(x) \gamma^{\mu} \ell_{\beta}(x)
$$

which corresponds to the Noether-current for the lepton flavor $\alpha$ for $\alpha=\beta$. We further define a time-dependent lepton-number matrix by

$$
n_{L \alpha \beta}(t)=\frac{1}{V} \int_{V} d^{3} x\left\langle J_{L \alpha \beta}^{0}(t, \mathbf{x})\right\rangle .
$$


The entries on the diagonal correspond to the lepton asymmetry in flavor $\alpha$ at time $t$ per volume $V$. The total lepton asymmetry per volume summed over all flavors is

$$
n_{L}(t)=\sum_{\alpha} n_{L \alpha \alpha}(t)
$$

which is independent of the choice of the flavor basis. The expectation value of the lepton current operator can be related to the lepton propagator,

$$
j_{L \alpha \beta}^{\mu}(x) \equiv\left\langle J_{L \alpha \beta}^{\mu}(x)\right\rangle=\left\langle\bar{\ell}_{\alpha}(x) \gamma^{\mu} \ell_{\beta}(x)\right\rangle=-\operatorname{tr}\left[\gamma_{\mu} S_{\ell}^{\alpha \beta}(x, x)\right] .
$$

In the presence of lepton-number violating processes, its divergence can be nonzero and may be written in the form:

$$
\begin{aligned}
& \partial_{\mu} j_{L \alpha \beta}^{\mu}(x)=-\operatorname{tr}\left[\gamma_{\mu}\left(\partial_{x}^{\mu}+\partial_{y}^{\mu}\right) S_{\ell}^{\alpha \beta}(x, y)\right]_{x=y} \\
& \quad=-\operatorname{tr}\left[\partial_{x} S_{\ell}^{\alpha \beta}(x, y)+S_{\ell}^{\alpha \beta}(x, y) \partial_{y}\right]_{x=y} \\
& \quad=-\int_{\mathcal{C}} d^{4} z \operatorname{tr}\left[S_{\ell_{0}^{-1}}^{\alpha \gamma}(x, z) S_{\ell}^{\gamma \beta}(z, y)-S_{\ell}^{\alpha \gamma}(x, z) S_{\ell_{0}}^{-1 \gamma \beta}(z, y)\right]_{x=y} .
\end{aligned}
$$

In the last line we have inserted the free inverse lepton propagator (23), $S_{\ell_{0}^{-1}}{ }^{\alpha \beta}(x, y)=\delta^{\alpha \beta} \not_{x} \delta_{\mathcal{C}}(x-y) P_{L}$, and used the chiral symmetry relations $S_{\ell}^{\alpha \beta}=P_{L} S_{\ell}^{\alpha \beta}=S_{\ell}^{\alpha \beta} P_{R}$. Now, one can replace the free inverse propagator $S_{\ell_{0}}^{-1}$ on the right-hand side using the Schwinger-Dyson equation $S_{\ell}^{-1}=$ $S_{\ell_{0}^{-1}}-\Sigma_{\ell}$. The parts containing $S_{\ell}^{-1} S_{\ell}$ do cancel, and we obtain the following equation of motion for the lepton current:

$$
\partial_{\mu} j_{L \alpha \beta}^{\mu}(x)=-\int_{\mathcal{C}} d^{4} z \operatorname{tr}\left[\Sigma_{\ell}^{\alpha \gamma}(x, z) S_{\ell}^{\gamma \beta}(z, x)-S_{\ell}^{\alpha \gamma}(x, z) \Sigma_{\ell}^{\gamma \beta}(z, x)\right] .
$$

From this, one can immediately derive an equation of motion for the lepton asymmetry (assuming a spatially homogeneous system for which $\partial_{\mu} j^{\mu}=\partial_{0} j^{0}$ ),

$$
\frac{d n_{L_{\alpha \beta}}(t)}{d t}=-\frac{1}{V} \int d^{3} x \int_{\mathcal{C}} d^{4} y \operatorname{tr}\left[\Sigma_{\ell}^{\alpha \gamma}(x, y) S_{\ell}^{\gamma \beta}(y, x)-S_{\ell}^{\alpha \gamma}(x, y) \Sigma_{\ell}^{\gamma \beta}(y, x)\right],
$$

where $x=(t, \mathbf{x})$. This can be considered as the master equation for the quantum-mechanical treatment of leptogenesis. For future reference, we also remark that one can rewrite the equation equivalently as

$$
\frac{d n_{L \alpha \beta}(t)}{d t}=-\frac{1}{V} \int d^{3} x \int_{\mathcal{C}} d^{4} y \operatorname{tr}\left[\Sigma_{\ell}^{\alpha \gamma}(x, y) S_{\ell}^{\gamma \beta}(y, x)-\bar{\Sigma}_{\ell}^{\beta \gamma}(x, y) \bar{S}_{\ell}^{\gamma \alpha}(y, x)\right] \text {, }
$$

where we have used that $\operatorname{tr} \bar{\Sigma}_{\ell}^{\beta \gamma}(\bar{x}, \bar{y}) \bar{S}_{\ell}^{\gamma \alpha}(\bar{y}, \bar{x})=\operatorname{tr} S_{\ell}^{\alpha \gamma}(x, y) \Sigma_{\ell}^{\gamma \beta}(y, x)$, and substituted $\mathbf{x} \rightarrow-\mathbf{x}$ and $\mathbf{y} \rightarrow-\mathbf{y}$ in the spatial integrals for the second term.

The 'master' equation (47) can be rewritten in a more explicit form by inserting the decomposition into the statistical and spectral components. In addition, for a spatially homogeneous system the two-point functions depend only on the difference of the spatial coordinates $\mathbf{x}-\mathbf{y}$. Then it is convenient to 
switch to Fourier space in the spatial coordinates,

$$
\begin{aligned}
\frac{d n_{L \alpha \beta}(t)}{d t}=i \int \frac{d^{3} p}{(2 \pi)^{3}} \int_{0}^{t} d t^{\prime} \\
\times \operatorname{tr}\left[\Sigma_{\ell \rho \mathbf{p}}^{\alpha \gamma}\left(t, t^{\prime}\right) S_{\ell F \mathbf{p}}^{\gamma \beta}\left(t^{\prime}, t\right)-\Sigma_{\ell F \mathbf{p}}^{\alpha \gamma}\left(t, t^{\prime}\right) S_{\ell_{\rho \mathbf{p}}^{\gamma \beta}}^{\gamma \beta}\left(t^{\prime}, t\right)\right. \\
\left.\quad-S_{\ell \rho \mathbf{p}}^{\alpha \gamma}\left(t, t^{\prime}\right) \Sigma_{\ell F \mathbf{p}}^{\gamma \beta}\left(t^{\prime}, t\right)+S_{\ell F \mathbf{p}}^{\alpha \gamma}\left(t, t^{\prime}\right) \Sigma_{\ell_{\rho \mathbf{p}}^{\gamma \beta}}^{\gamma \beta}\left(t^{\prime}, t\right)\right] .
\end{aligned}
$$

By integrating the upper equation with respect to $t$, one can easily obtain the time-evolution of the lepton asymmetry $n_{L \alpha \beta}(t)$,

$$
\begin{aligned}
n_{L \alpha \beta}(t)=i \int \frac{d^{3} p}{(2 \pi)^{3}} \int_{0}^{t} d t^{\prime} \int_{0}^{t^{\prime}} d t^{\prime \prime} \\
\times \operatorname{tr}\left[\Sigma_{\ell \rho \mathbf{p}}^{\alpha \gamma}\left(t^{\prime}, t^{\prime \prime}\right) S_{\ell F \mathbf{p}}^{\gamma \beta}\left(t^{\prime \prime}, t^{\prime}\right)-\Sigma_{\ell F \mathbf{p}}^{\alpha \gamma}\left(t^{\prime}, t^{\prime \prime}\right) S_{\ell_{\rho \mathbf{p}}^{\gamma \beta}}^{\gamma \beta}\left(t^{\prime \prime}, t^{\prime}\right)\right. \\
\left.\quad-S_{\ell_{\rho} \mathbf{p}}^{\alpha \gamma}\left(t^{\prime}, t^{\prime \prime}\right) \Sigma_{\ell F \mathbf{p}}^{\gamma \beta}\left(t^{\prime \prime}, t^{\prime}\right)+S_{\ell_{F} \mathbf{p}}^{\alpha \gamma}\left(t^{\prime}, t^{\prime \prime}\right) \Sigma_{\ell_{\rho \mathbf{p}}^{\gamma \beta}}^{\gamma \beta}\left(t^{\prime \prime}, t^{\prime}\right)\right] .
\end{aligned}
$$

If the lepton propagator is approximately flavor-diagonal, $S_{\ell}^{\alpha \beta}=\delta^{\alpha \beta} S_{\ell}$, it is easy to see that the integrand is symmetric with respect to $t^{\prime} \leftrightarrow t^{\prime \prime}$. Using that $\int_{0}^{t} d t^{\prime} \int_{0}^{t^{\prime}} d t^{\prime \prime}\left(f\left(t^{\prime}, t^{\prime \prime}\right)+f\left(t^{\prime \prime}, t^{\prime}\right)\right)=\int_{0}^{t} d t^{\prime} \int_{0}^{t} d t^{\prime \prime} f\left(t^{\prime}, t^{\prime \prime}\right)$, it is possible to obtain a simplified equation,

$$
\begin{aligned}
n_{L \alpha \beta}(t)=i \int \frac{d^{3} p}{(2 \pi)^{3}} \int_{0}^{t} d t^{\prime} \int_{0}^{t} d t^{\prime \prime} \operatorname{tr}[ & {\left[\sum_{\ell \rho \mathbf{p}}^{\alpha \beta}\left(t^{\prime}, t^{\prime \prime}\right) S_{\ell F \mathbf{p}}\left(t^{\prime \prime}, t^{\prime}\right)\right.} \\
& \left.-\Sigma_{\ell F \mathbf{p}}^{\alpha \beta}\left(t^{\prime}, t^{\prime \prime}\right) S_{\ell \rho \mathbf{p}}\left(t^{\prime \prime}, t^{\prime}\right)\right] .
\end{aligned}
$$

This equation essentially agrees with Eq. (20) of [36]. However, we find it more convenient to use Eq. (48) in the following.

\subsection{Generation of an asymmetry}

In the following, we will explicitly compute the time-evolution of the lepton asymmetry in the full two-time approach using the two-loop truncation of the 2PI effective action. For simplicity, we will neglect washout effects and the expansion of the universe for the moment. Furthermore, we will assume that the generated asymmetry is small, such that one can expand around a $C P$ symmetric state. For that purpose, we parameterize the two-point functions and their $C P$-conjugates as

$$
\begin{aligned}
& D(x, y)=D^{s}(x, y)+\frac{1}{2} \delta D(x, y), \\
& \bar{D}(x, y)=D^{s}(x, y)-\frac{1}{2} \delta D(x, y),
\end{aligned}
$$

where $D \in\left\{\Delta_{\phi}, S_{\ell}\right\}$. For a $C P$-symmetric state (i.e. with no lepton asymmetry), $\delta D$ vanishes. Inserting this parameterization into the equation of motion (48) for the lepton asymmetry, and expanding in $\delta D$, yields at leading order

$$
\frac{d n_{L_{\alpha \beta}}(t)}{d t}=-\frac{1}{V} \int d^{3} x \int_{\mathcal{C}} d^{4} y \operatorname{tr}\left[\left.\left(\Sigma_{\ell}^{\alpha \beta}(x, y)-\bar{\Sigma}_{\ell}^{\beta \alpha}(x, y)\right)\right|_{s} S_{\ell}^{s}(y, x)\right],
$$


where we have assumed $S_{\ell}^{\alpha \beta} \approx \delta^{\alpha \beta} S_{\ell}$. The propagators entering the selfenergies are to be evaluated with the symmetric parts of the propagators, $D^{s}$, as indicated by the subscript. The washout terms would contribute at next-to leading order in $\delta D$ (see Appendix B).

Now we are ready to insert the explicit one-loop expressions for the lepton self-energy (34) and (39). This yields the following equation for the lepton asymmetry:

$$
\frac{d n_{L \alpha \beta}(t)}{d t}=\frac{h_{\alpha i} h_{j \beta}^{\dagger}}{V} \int d^{3} x \int_{\mathcal{C}} d^{4} y \operatorname{tr}\left[P_{R}\left(S^{i j}(x, y)-\bar{S}^{j i}(x, y)\right) P_{L} S_{\ell \phi}(y, x)\right] .
$$

For brevity, we have dropped the superscript 's' on lepton and Higgs propagators. By performing manipulations similar to the ones above,

$$
\begin{aligned}
n_{L \alpha \beta}(t)=-i h_{\alpha i} h_{j \beta}^{\dagger} \int \frac{d^{3} p}{(2 \pi)^{3}} \int_{0}^{t} d t^{\prime} \int_{0}^{t} d t^{\prime \prime} \\
\operatorname{tr}\left[P_{R}\left(S_{\rho \mathbf{p}}^{i j}\left(t^{\prime}, t^{\prime \prime}\right)-\bar{S}_{\rho \mathbf{p}}^{j i}\left(t^{\prime}, t^{\prime \prime}\right)\right) P_{L} S_{\ell \phi_{F} \mathbf{p}}\left(t^{\prime \prime}, t^{\prime}\right)\right. \\
\left.-P_{R}\left(S_{F \mathbf{p}}^{i j}\left(t^{\prime}, t^{\prime \prime}\right)-\bar{S}_{F \mathbf{p}}^{j i}\left(t^{\prime}, t^{\prime \prime}\right)\right) P_{L} S_{\ell \phi_{\rho \mathbf{p}}}\left(t^{\prime \prime}, t^{\prime}\right)\right] .
\end{aligned}
$$

This equation provides a quantum field theoretical expression for the lepton asymmetry that is generated from the wave or self-energy type contributions. It is appropriate to study the resonant case, where these contributions dominate. As input, it requires the two-point functions for the lepton, Higgs and Majorana neutrino fields. Since leptons and the Higgs interact much stronger than the Majorana neutrino, it is often assumed that they are in thermal equilibrium. Thus, one needs to provide (at least) the equilibrium expressions for the lepton and Higgs propagators, as well as a non-equilibrium Majorana propagator. In particular, the information on the $C P$ violation is encoded in the off-diagonal components of the Majorana propagator. In the following, we will evaluate the lepton asymmetry for different choices for these propagators, that correspond to different approximation levels, and enable a comparison to the conventional Boltzmann result.

\subsection{Asymmetry in a thermal bath}

In the following, we will derive an expression for the lepton asymmetry assuming that lepton and the Higgs fields can be treated as a thermal bath, and that the back-reaction can be neglected. This setup has been considered in Refs. [36, 37] for a hierarchical neutrino spectrum. Concretely, this means that we assume that the two-point functions for lepton and the Higgs fields are time-translation invariant,

$$
\begin{aligned}
S_{\ell}(x, y) & =S_{\ell}^{t h}(x-y), \\
\Delta_{\phi}(x, y) & =\Delta_{\phi}^{t h}(x-y) .
\end{aligned}
$$

In the following we will explore the consequences of this assumption. The most important one is that it is possible to obtain an analytical solution of the Kadanoff-Baym equations for the Majorana neutrino fields, which will be discussed in the following. 


\section{Non-equilibrium Majorana Propagator}

As a first step, we derive a Kadanoff-Baym equation for the retarded and advanced propagators

$$
\begin{aligned}
& S_{R}(x, y)=\Theta\left(x^{0}-y^{0}\right) S_{\rho}(x, y), \\
& S_{A}(x, y)=-\Theta\left(y^{0}-x^{0}\right) S_{\rho}(x, y) .
\end{aligned}
$$

First, we apply the differential operator that appears on the left-hand side of the Kadanoff-Baym Eq. (31a) to the first line,

$$
\begin{aligned}
& \left(i \not \not_{x}-M-\delta \Sigma_{N}(x)\right) S_{R}(x, y) \\
& \quad=i \gamma_{0} \delta\left(x^{0}-y^{0}\right) S_{\rho}(x, y)+\Theta\left(x^{0}-y^{0}\right)\left(i \not \partial_{x}-M-\delta \Sigma_{N}(x)\right) S_{\rho}(x, y) .
\end{aligned}
$$

Here we have used that the spectral function at equal times is determined by the commutation relations of the Majorana neutrino fields,

$$
\left.S_{\rho}^{i j}(x, y)\right|_{x^{0}=y^{0}}=i\left\langle\left\{N_{i}(x), \bar{N}_{j}(y)\right\}\right\rangle_{x^{0}=y^{0}}=i \gamma_{0} \delta^{i j} \delta(\mathbf{x}-\mathbf{y}) .
$$

Using the Kadanoff-Baym Eq. (31a) for the spectral function, we obtain

$$
\begin{aligned}
& \left(i \not \not_{x}-M-\delta \Sigma_{N}(x)\right) S_{R}(x, y) \\
& \quad=-\delta^{i j} \delta(x-y)+\Theta\left(x^{0}-y^{0}\right) \int_{y^{0}}^{x^{0}} d^{4} z \Sigma_{N \rho}(x, z) S_{\rho}(z, y) .
\end{aligned}
$$

Next we rewrite the memory integral using the identity

$$
\int_{y^{0}}^{x^{0}} d^{4} z=\int d^{4} z\left[\Theta\left(x^{0}-z^{0}\right) \Theta\left(z^{0}-y^{0}\right)-\Theta\left(y^{0}-z^{0}\right) \Theta\left(z^{0}-x^{0}\right)\right] .
$$

where $\int d^{4} z=\int d z^{0} \int d^{3} z$ denotes a time-integration over the whole real axis and over spatial coordinates. Due to the factor $\Theta\left(x^{0}-y^{0}\right)$ in Eq. (59), only the first term contributes. Inserting this relation yields a Kadanoff-Baym equation for the retarded propagator. The equation for the advanced propagator can be derived analogously. They can be summarized as

$$
\begin{aligned}
& \left(i \not \not_{x}-M-\delta \Sigma_{N}(x)\right) S_{R(A)}(x, y) \\
& \quad=\quad-\delta^{i j} \delta(x-y)+\int d^{4} z \Sigma_{N R(A)}(x, z) S_{R(A)}(z, y) .
\end{aligned}
$$

For thermal lepton and Higgs fields, the self-energies are translation invariant and depend only on the relative coordinate $s=x-y$ (note that $\delta \Sigma_{N}(x)=$ $\delta \Sigma_{N}(x+a)$ ). This implies that the equations for retarded and advanced propagators become invariant under the translation $x \rightarrow x^{\prime}=x+a, y \rightarrow y^{\prime}=y+a$. Furthermore, their initial conditions are fixed by the equal-time commutation relations, as for the spectral function. Consequently, the retarded and advanced propagators themselves are translation invariant, $S_{R(A)}(x, y)=S_{R(A)}\left(x^{\prime}, y^{\prime}\right)$. This means that they can depend only on the relative coordinate as well, i.e. $S_{R(A)}(x, y)=S_{R(A)}(x-y)$. For functions that depend on $x-y$, it is convenient to switch to momentum space. We define the Fourier transformation by

$$
G(x-y)=\int \frac{d^{4} p}{(2 \pi)^{4}} e^{-i p(x-y)} G(p) .
$$


The Fourier transformed equation for retarded and advanced propagators reads

$$
\left[\left(\not p-M_{i}\right) \delta^{i k}-\delta \Sigma_{N}^{i k}(p)-\Sigma_{N}^{i k(A)}(p)\right] S_{R(A)}^{k j}(p)=-\delta^{i j} .
$$

This is an algebraic version of the Schwinger-Dyson equation, with Dirac as well as flavor matrix structure. The interaction with the thermal bath of lepton and Higgs is encoded in the self-energy. The sum of the retarded or advanced self-energy and the counterterm contribution yields renormalized expressions for the self-energy.

Now we can return to the Kadanoff-Baym equations for the spectral and statistical Majorana propagators, Eq. (31a). The spectral function is directly related to the retarded and advanced propagators,

$$
S_{\rho}(x-y)=S_{R}(x-y)-S_{A}(x-y) .
$$

Consequently it is also translation invariant.

The Kadanoff-Baym equation for the statistical propagator, Eq. (31a), can be written as

$$
\begin{array}{r}
\int_{0}^{\infty} d^{4} z\left[\left(\left(\not \not \partial_{x}-M_{i}\right) \delta^{i k}-\delta \Sigma_{N}^{i k}(x)\right) \delta(x-z)-\Sigma_{N R}^{i k}(x, z)\right] S_{F}^{k j}(z, y) \\
=\int_{0}^{\infty} d^{4} z \Sigma_{N}^{i k}(x, z) S_{A}^{k j}(z, y) .
\end{array}
$$

In this form, the homogeneous part is written in the first line and the inhomogeneous part in the second one. The solution can be written as the sum of a special solution of the inhomogeneous equations plus the general solution of the homogeneous equation. For the former we make the ansatz

$$
S_{F}^{i j}(x, y)_{\text {inhom }}=-\int_{0}^{\infty} d^{4} u S_{R}^{i k}(x, u) \int_{0}^{\infty} d^{4} z \Sigma_{N F}^{k l}(u, z) S_{A}^{l j}(z, y) .
$$

The application of the operator in square brackets in Eq. (64) will turn the retarded propagator into a delta function. Then integration over $u$ becomes trivial and yields the right-hand side of the Kadanoff-Baym equation, as required.

Next, we consider the homogeneous equation, i.e. we set the right-hand side in Eq. (64) to zero. The general solution will depend on the initial conditions. Since only the first derivative appears, the general solutions can be completely specified by the initial value of the propagator itself, $\left.S_{F}^{i j}(x, y)\right|_{x^{0}=y^{0}=0}$. Here, we make the ansatz,

$$
S_{F}^{i j}(x, y)_{h o m}=-\int d^{3} u S_{R}^{i k}(x,(0, \mathbf{u})) \int d^{3} v A^{k l}(\mathbf{u}, \mathbf{v}) S_{A}^{l j}((0, \mathbf{v}), y),
$$

where $A^{k l}(\mathbf{u}, \mathbf{v})$ is a free function. Using again the equation of motion for the retarded propagator, one can see that this ansatz indeed solves the homogeneous equation (except for delta-functions located at the initial time surface; these determine the initial conditions but do not influence the validity of the solution). Also, it is important that the dependence on $y$ solves the hermitian conjugated Kadanoff-Baym equation, as required. The initial condition to which the ansatz corresponds can be extracted by letting $x^{0}, y^{0} \rightarrow 0$ from above. In this case we 
can replace the retarded and advanced functions by the spectral function, and use its equal-time limit. This yields

$$
\left.S_{F}^{i j}(x, y)_{h o m}\right|_{x^{0}=y^{0}=0}=i \gamma_{0} A^{i j}(\mathbf{x}, \mathbf{y}) i \gamma_{0} .
$$

Thus there is a simple one-to-one correspondence between the function $A^{i j}(\mathbf{x}, \mathbf{y})$ and the initial condition of the statistical propagator. The general solution is given by the sum of both contributions,

$$
S_{F}^{i j}(x, y)=S_{F}^{i j}(x, y)_{i n h o m}+S_{F}^{i j}(x, y)_{h o m} .
$$

This is a formal solution, which is valid even if lepton and Higgs were not in equilibrium. However, when they are treated as a thermal bath, the retarded and advanced functions as well as the self-energy depend only on the time-differences. Thus, in a thermal bath, the integrand in the inhomogeneous contribution involves only time-translation invariant functions. Therefore, one might expect that the inhomogeneous contribution is itself time-translation invariant. However, this is not the case because of the lower integration limits, which start at the initial time $t=0$. The deeper reason behind this is that standard KadanoffBaym equations rely on a hidden assumption. Namely, it is implicitly assumed that all the higher correlation functions (three-point, four-point function etc.) vanish at $t=0$, i.e. that the system is in a Gaussian state at $t=0$. Then, higher correlations have to build up, which is reflected in the finite integration range starting at $t=0$. Physically, we expect that higher correlations are present at any time. A simple way to incorporate these is to consider also the evolution of the system at times 'before' the initial time, i.e. for $t<0$. Technically, this means that all integrations start at $t=-\infty$ instead of $t=0$,

$$
S_{F}^{i j}(x, y)_{i n h o m}=-\int_{-\infty}^{\infty} d^{4} u \int_{-\infty}^{\infty} d^{4} v\left[S_{R}^{i k}(x, u) \Sigma_{N F}^{k l}(u, v) S_{A}^{l j}(v, y)\right] .
$$

Then, the inhomogeneous contribution will no longer vanish at $x^{0}=y^{0}=0$, but will be determined by the pre-evolution of the system (i.e. at times $x^{0}, y^{0}<0$ ). On the other hand, the ansatz Eq. (66) still represents a valid solution of the homogeneous equation for non-zero time arguments. It describes a Gaussian deviation from equilibrium occurring at $x^{0}=y^{0}=0$, and can be traced back to an external source term, that is also present within the usual Gaussian framework and that originates from the contribution of the density matrix to the 2PI effective action [62,63].

Let us now revisit what happens when lepton and the Higgs are treated as a thermal bath. Then, since retarded and advanced propagators as well as the self-energy depend only on the difference of the time arguments, and the integration range is now over the whole real axis, the inhomogeneous solution itself will be time-translation invariant, i.e.

$$
\begin{aligned}
S_{F}^{i j}(x, y)_{\text {inhom }} & =-\int_{-\infty}^{\infty} d^{4} u \int_{-\infty}^{\infty} d^{4} v\left[S_{R}^{i k}(x-u) \Sigma_{N}^{k l}(u-v) S_{A}^{l j}(v-y)\right] \\
& =S_{F}^{i j}(x-y)_{\text {inhom }}=S_{F}^{i j t h}(x-y) .
\end{aligned}
$$

This means that, in a thermal bath, the inhomogeneous part simply corresponds to the thermal equilibrium propagator. The deviation from equilibrium is therefore described by the homogeneous contribution Eq. (66). In particular, from 
Eq. (67) it follows that the function $A$ appearing in Eq. (66) specifies the deviation from equilibrium at the 'initial' time $x^{0}=y^{0}=0$,

$$
\begin{aligned}
\left.\Delta S_{F}^{i j}(x, y)\right|_{x^{0}=y^{0}=0} & =\left(S_{F}^{i j}(x, y)-S_{F}^{i j t h}(x-y)\right)_{x^{0}=y^{0}=0} \\
& =i \gamma_{0} A^{i j}(\mathbf{x}, \mathbf{y}) i \gamma_{0} .
\end{aligned}
$$

Since we are interested mainly in spatially homogeneous systems, it is convenient to switch to spatial momentum space, but to retain the dependence on time. In particular the function $A$ fulfills $A^{i j}(\mathbf{x}, \mathbf{y})=A^{i j}(\mathbf{x}-\mathbf{y})$. Then the formal solution for the statistical propagator can be summarized as

$$
\begin{aligned}
S_{F \mathbf{p}}^{i j}\left(t, t^{\prime}\right)= & -\int_{-\infty}^{\infty} d u \int_{-\infty}^{\infty} d v\left[S_{R \mathbf{p}}^{i k}(t, u) \Sigma_{N F \mathbf{p}}^{k l}(u, v) S_{A \mathbf{p}}^{l j}\left(v, t^{\prime}\right)\right] \\
& -S_{R \mathbf{p}}^{i k}(t, 0) A_{\mathbf{p}}^{i j} S_{A \mathbf{p}}^{l j}\left(0, t^{\prime}\right) .
\end{aligned}
$$

As discussed above, in a thermal bath the inhomogeneous part of the solution yields the thermal propagator, and the homogeneous part describes the deviation from equilibrium. The solution for the statistical propagator can thus be written as

$$
\begin{aligned}
S_{F \mathbf{p}}^{i j}\left(t, t^{\prime}\right) & =S_{F \mathbf{p}}^{i j t h}\left(t-t^{\prime}\right) \\
& -S_{R \mathbf{p}}^{i k}(t) i \gamma_{0} \Delta S_{F \mathbf{p}}^{k l}(0,0) i \gamma_{0} S_{A \mathbf{p}}^{l j}\left(-t^{\prime}\right) .
\end{aligned}
$$

Due to the retarded and advanced functions, the second line is non-zero only when $t>0$ and $t^{\prime}>0$. This means that it describes the impact of a distortion that has occurred at time $t=t^{\prime}=0$. In other words, it describes how the system reacts and how the distortion is propagated to positive times $t, t^{\prime}>0$. We emphasize that there were no assumptions necessary that would limit the size of the 'distortion' described by $\Delta S_{F \mathbf{p}}^{k l}(0,0)$. This means that the equation is valid also for large deviations from equilibrium, as long as the assumption of a thermal bath is justified. For example, for 'zero initial abundance', the Majorana propagator at $t=t^{\prime}=0$ is the vacuum one, $S_{F \mathbf{p}}^{k l}(0,0)=S_{F \mathbf{p}}^{k l v a c}(0)$. This means one has to choose $\Delta S_{F \mathbf{p}}^{k l}(0,0)=S_{F}^{k l} \mathbf{p}$ vac $(0)-S_{F \mathbf{p}}^{k l}(0)$.

\section{Asymmetry for thermal lepton and Higgs}

The starting point of our calculation of the lepton asymmetry is the quantum field theoretical expression Eq. (55). We insert the thermal lepton and Higgs propagators, and write the Majorana propagator in the form

$$
S_{F \mathbf{p}}^{i j}\left(t, t^{\prime}\right)=S_{F \mathbf{p}}^{i j h}\left(t-t^{\prime}\right)+\Delta S_{F \mathbf{p}}^{i j}\left(t, t^{\prime}\right) .
$$

Furthermore, we extend the lower limits of integration in Eq. (55) to $-\infty$ in order to take higher correlations at the 'initial' time $t=0$ into account, as explained above. Then the contribution to the asymmetry that involves only equilibrium propagators vanishes, because the lepton asymmetry is zero in thermal equilibrium. Next, we insert the solution Eq. (73) of the Kadanoff-Baym equation for the statistical propagator, which can be written as

$$
\begin{aligned}
\Delta S_{F \mathbf{p}}^{i j}\left(t, t^{\prime}\right) & =-S_{R \mathbf{p}}^{i k}(t) i \gamma_{0} \Delta S_{F \mathbf{p}}^{k l}(0,0) i \gamma_{0} S_{A \mathbf{p}}^{l j}\left(-t^{\prime}\right) \\
& =\Theta(t) \Theta\left(t^{\prime}\right) S_{\rho \mathbf{p}}^{i k}(t) i \gamma_{0} \Delta S_{F \mathbf{p}}^{k l}(0,0) i \gamma_{0} S_{\rho \mathbf{p}}^{l j}\left(-t^{\prime}\right) .
\end{aligned}
$$


It is non-zero only for $t>0$ and $t^{\prime}>0$. This means that the lower limits of integration in Eq. (55) are again zero. Note that it was nevertheless important to extend them in the first place, because otherwise the 'equilibrium' contribution would not have vanished due to unwanted correlation build-up effects. Altogether, we arrive at the following expression for the lepton asymmetry in a thermal bath:

$$
\begin{aligned}
n_{L \alpha \beta}(t)= & i h_{\alpha i} h_{j \beta}^{\dagger} \int \frac{d^{3} p}{(2 \pi)^{3}} \int_{0}^{t} d t^{\prime} \int_{0}^{t} d t^{\prime \prime} \\
& \operatorname{tr}\left[P_{R}\left(\Delta S_{F \mathbf{p}}^{i j}\left(t^{\prime}, t^{\prime \prime}\right)-\Delta \bar{S}_{F \mathbf{p}}^{j i}\left(t^{\prime}, t^{\prime \prime}\right)\right) P_{L} S_{\ell \phi} \mathbf{p}\left(t^{\prime \prime}-t^{\prime}\right)\right] .
\end{aligned}
$$

\section{Weakly interacting thermal bath}

A case of particular interest arises when the interactions of lepton and the Higgs are strong enough to maintain thermal equilibrium, but at the same time weak enough to justify the use of free thermal propagators for their two-point functions. In the following we will explore the consequences of this assumption, and we postpone the discussion whether this assumption is justified within the SM. The case of free thermal propagators is also interesting because it enables a rather direct comparison with the Boltzmann result.

The free thermal propagators for the Higgs and lepton fields in the time/momentum representation are given by

$$
\begin{aligned}
\Delta_{\phi F \mathbf{k}}^{e q}\left(t-t^{\prime}\right) & =\frac{1+2 f_{\phi}^{e q}(k)}{2 k} \cos \left(k\left(t-t^{\prime}\right)\right), \\
\Delta_{\phi \rho \mathbf{k}}^{e q}\left(t-t^{\prime}\right) & =\frac{1}{k} \sin \left(k\left(t-t^{\prime}\right)\right),
\end{aligned}
$$

and

$$
\begin{aligned}
S_{\ell F \mathbf{k}}^{e q}\left(t-t^{\prime}\right) & =\frac{1-2 f_{\ell}^{e q}(k)}{2}\left\{\frac{-\mathbf{k} \cdot \gamma}{k} \cos \left(k\left(t-t^{\prime}\right)\right)-i \gamma_{0} \sin \left(k\left(t-t^{\prime}\right)\right)\right\}, \\
S_{\ell \rho \mathbf{k}}^{e q}\left(t-t^{\prime}\right) & =\left\{\frac{-\mathbf{k} \cdot \gamma}{k} \sin \left(k\left(t-t^{\prime}\right)\right)+i \gamma_{0} \cos \left(k\left(t-t^{\prime}\right)\right)\right\}
\end{aligned}
$$

where $k=|\mathbf{k}|, f_{\phi}^{e q}(k)=1 /\left(e^{k / T}-1\right)$, and $f_{\ell}^{e q}(k)=1 /\left(e^{k / T}+1\right)$. The chiral propagator is obtained by multiplying with the projector $P_{L}$ from the left. Note that the correct chiral structure is nevertheless taken into account by the projectors $P_{L / R}$ contained in the self-energies. In addition, similar to Ref. [37], by considering damped Breit-Wigner propagators we qualitatively take into account the effect of a thermal width in the lepton-Higgs-loop $S_{\ell \phi_{\rho}}$ appearing in Eq. (76)

$$
\begin{aligned}
\Delta_{\phi F(\rho) \mathbf{k}}^{e q}\left(t-t^{\prime}\right) & \rightarrow \Delta_{\phi F(\rho) \mathbf{k}}^{e q}\left(t-t^{\prime}\right) e^{-\Gamma_{\phi}\left|t-t^{\prime}\right| / 2} \\
S_{\ell F(\rho) \mathbf{k}}^{e q}\left(t-t^{\prime}\right) & \rightarrow S_{\ell F(\rho) \mathbf{k}}^{e q}\left(t-t^{\prime}\right) e^{-\Gamma_{\ell}\left|t-t^{\prime}\right| / 2}
\end{aligned}
$$

Evaluating the lepton-Higgs-loop $S_{\ell \phi \rho}$ defined in Eq. (37a) with the thermal 
lepton and Higgs propagators specified above yields

$$
\begin{aligned}
& S_{\ell \phi_{\rho} \mathbf{p}}^{e q}\left(t-t^{\prime}\right)=\int \frac{d^{3} q}{(2 \pi)^{3}}\left[S_{\ell \rho \mathbf{k}}^{e q}\left(t-t^{\prime}\right) \Delta_{\phi F \mathbf{q}}^{e q}\left(t-t^{\prime}\right)+S_{\ell F \mathbf{k}}^{e q}\left(t-t^{\prime}\right) \Delta_{\phi \rho \mathbf{q}}^{e q}\left(t-t^{\prime}\right)\right] \\
& =\int \frac{d^{3} q}{(2 \pi)^{3} 2 q} e^{-\Gamma_{\ell \phi}|u| / 2}\left\{S_{\ell \phi_{\rho}}^{++} e^{i(k+q) u}+S_{\ell \phi_{\rho}^{-}}^{-} e^{-i(k+q) u}\right. \\
& \left.+S_{\ell \phi \rho}^{+-} e^{i(k-q) u}+S_{\ell \phi}^{-+} e^{-i(k-q) u}\right\} .
\end{aligned}
$$

The coefficients introduced in the last line are given by

$$
\begin{aligned}
S_{\ell \phi \rho}^{ \pm \pm} & =\frac{i}{2 k}\left(k \gamma_{0} \pm \mathbf{k} \gamma\right) f_{\ell \phi}(k, q), \\
S_{\ell \phi_{\rho}^{\mp}}^{ \pm} & =\frac{i}{2 k}\left(k \gamma_{0} \pm \mathbf{k} \gamma\right)\left(f_{\phi}(q)+f_{\ell}(k)\right),
\end{aligned}
$$

where $\mathbf{k} \equiv \mathbf{p}-\mathbf{q}, q=|\mathbf{q}|$ and $k=|\mathbf{k}|, \Gamma_{\ell \phi}=\Gamma_{\ell}+\Gamma_{\phi}$, and

$$
f_{\ell \phi}(k, q)=1+f_{\phi}(q)-f_{\ell}(k) .
$$

\section{Analytical Breit-Wigner approach}

The equation for the lepton asymmetry, Eq. (76), together with the expression (75) for the non-equilibrium evolution of the Majorana neutrinos in flavor space, and with the Schwinger-Dyson equations (62) for the retarded and advanced neutrino propagators can be solved numerically. Before discussing the numerical solution, it is however very useful to pursue an analytical approach. This is the content of this section.

We will proceed in three steps. First, we compute the retarded and advanced two-point functions for the Majorana neutrinos by employing a Breit-Wigner approximation, and taking the flavor matrix structure into account. This will allow us to identify effective pole masses and widths of the quasi-degenerate Majorana neutrino flavors. Then, we will use these propagators to determine a solution of the Kadanoff-Baym equation for the statistical propagator, which describes the non-equilibrium evolution of the Majorana neutrino fields. Finally, this solution will be used to compute the time-dependent lepton asymmetry produced by the interaction of the Majorana neutrinos with the thermal lepton and Higgs background.

\subsection{Retarded and advanced propagators}

The retarded and advanced Majorana neutrino two-point functions can be obtained by solving the algebraic Schwinger-Dyson equation (62) in momentum space. Since we are interested in the time-dependence, we will also perform a Fourier transformation.

In order to solve the Schwinger-Dyson equation (62), we insert the thermal lepton and Higgs propagators $(77,78)$ into the expression (36) for the Majorana neutrino self-energy. The self-energy can be written in the form

$$
\Sigma_{N_{R(A)}^{i j}}(p)=-2\left[\left(h^{\dagger} h\right)_{i j} P_{L}+\left(h^{\dagger} h\right)_{j i} P_{R}\right] S_{\ell \phi_{R(A)}}(p),
$$


where we have assumed even $C P$ parities $\eta_{i}=1$ for simplicity. The function $S_{\ell \phi_{R(A)}}(p)$ denotes the lepton-Higgs loop integral. It can be written as a sum of vacuum and thermal contributions. Using dimensional regularization, the former is given by

$$
S_{\ell \phi}^{v a c}(p)=\frac{1}{32 \pi^{2}}\left(\frac{1}{\epsilon}-\gamma_{E}+\ln (4 \pi)+2-\ln \left(\frac{\left|p^{2}\right|}{\mu^{2}}\right) \pm i \pi \Theta\left(p^{2}\right) \operatorname{sgn}\left(p_{0}\right)\right) \not p .
$$

The divergent contribution can be eliminated by introducing suitable mass and wave-function counterterms for the Majorana neutrino fields. If the difference between the Majorana mass parameters $M_{1,2}$ appearing in the Lagrangian is large compared to the decay width $\Gamma_{1,2}$, it is convenient to use an on-shell renormalization scheme. However, in the present work, we are interested in mass splittings of the order or even smaller than the width. In this case, we find it more convenient to use $\overline{M S}$ renormalization. This means that the mass parameters $M_{1,2}$ are not necessarily equal to the physical pole masses $M_{1,2}^{\text {pole }}$, and that the residues at the poles are not necessarily normalized. We will derive an equation for the pole masses in the following, and also properly take the residues into account in the computation of the lepton asymmetry. We stress that the lepton asymmetry is independent of the renormalization scheme, and thus we can choose it to our convenience.

Accordingly, we choose the wave-function and mass counterterms as

$$
\begin{aligned}
\delta Z_{i j} & =\frac{\left(h^{\dagger} h\right)_{j i}}{16 \pi^{2}}\left(\frac{1}{\epsilon}-\gamma_{E}+\ln (4 \pi)+2\right), \\
\delta M_{i j} & =-\frac{1}{2}\left(M_{i} \delta Z_{i j}+\delta Z_{j i} M_{j}\right) .
\end{aligned}
$$

In addition, it is convenient to choose the renormalization scale close to the mass scale of the Majorana neutrinos, such that the logarithm appearing in (82) is small. We will set $\mu=\left(M_{1}+M_{2}\right) / 2$ for definiteness. We have checked that our numerical results in section 5 are highly independent of the precise value in the range $M_{1}<\mu<M_{2}$.

In a $C P$-symmetric configuration and for positive $p^{2}$ and positive $p^{0}$ the thermal contribution to the lepton-Higgs loop is given by

$$
\begin{aligned}
& S_{\ell \phi R(A)}^{t h}(p)= \\
& \sum_{ \pm}\left[\int \frac{d^{3} k}{(2 \pi)^{3} 2 E_{k}} f_{\ell}\left(E_{k}\right) k_{ \pm} \frac{\mathcal{P}}{\left(p-k_{ \pm}\right)^{2}}-\int \frac{d^{3} q}{(2 \pi)^{3} 2 E_{q}} f_{\phi}\left(E_{q}\right)\left(\not p-\not q_{ \pm}\right) \frac{\mathcal{P}}{\left(p-q_{ \pm}\right)^{2}}\right] \\
& \pm \frac{i}{2} \int \frac{d^{3} k}{(2 \pi)^{3} 2 E_{k}} \frac{d^{3} q}{(2 \pi)^{3} 2 E_{q}}(2 \pi)^{4} \delta(p-q-k) \not k\left[f_{\phi}\left(E_{q}\right)-f_{\ell}\left(E_{k}\right)\right],
\end{aligned}
$$

where, to shorten the notation, we have introduced $k_{ \pm}=\left( \pm E_{k}, \mathbf{k}\right)$ and $q_{ \pm}=$ $\left( \pm E_{q}, \mathbf{q}\right)$. For negative $p^{0}$ the loop momentum $k=\left(E_{k}, \mathbf{k}\right)$ in the second line of (84) should be replaced with $k=\left(E_{k},-\mathbf{k}\right)$. For the analytical treatment, we will confine ourselves to the regime for which the Majorana neutrinos can be treated as non-relativistic when the lepton asymmetry is produced. In this case, the thermal contribution can be approximated by

$$
S_{\ell \phi}^{t h}(A)(p) \simeq-T^{2} \not p /\left(12 p^{2}\right) \pm 2 i \not p /\left(e^{\left|p_{0}\right| / T}-1\right) \Theta\left(p^{2}\right) \operatorname{sgn}\left(p_{0}\right) /(32 \pi) .
$$


The first term describes a thermal mass shift, while the second one encodes the finite width. For the analytical treatment it is convenient to change the renormalization prescription in order to take the leading effect of the thermal mass shift into account, $\delta Z_{i j} \rightarrow \delta Z_{i j}+\delta Z_{i j}(T)$ with $\delta Z_{i j}(T) \equiv\left(h^{\dagger} h\right)_{j i} T^{2} /\left(12 \mu^{2}\right)$. The relation between the mass- and Yukawa coupling matrices at zero and finite temperature is

$$
\begin{aligned}
M(T) & =\left(P_{L} Z(T)^{T}+P_{R} Z(T)^{\dagger}\right) M(T=0)\left(P_{L} Z(T)+P_{R} Z(T)^{*}\right)(86 \mathrm{a}) \\
\left(h^{\dagger} h\right)(T) & =Z(T)^{T}\left(h^{\dagger} h\right)(T=0) Z(T)^{*},
\end{aligned}
$$

where $Z_{i j}(T) \equiv\left(\delta_{i k}+\delta Z_{i k}(T)\right) V_{k j}(T)$. Here $V(T)$ is a unitary matrix in flavor space that can be adjusted such that the mass matrix at finite temperature is diagonal and has real and positive entries, like the one at zero temperature. In the following, all quantities refer to the finite temperature values. We stress again that the lepton asymmetry is unaffected by the field rescaling, i.e. we would obtain identical results when setting $\delta Z_{i j}(T)$ to zero and using the vacuum parameters instead. The rescaling is performed for computational convenience.

Altogether, when inserting the vacuum and thermal contributions to the self-energy as well as the counterterms into the Schwinger-Dyson equation (62), we obtain the following renormalized equations for the retarded and advanced propagators:

$$
\begin{aligned}
& {\left[\left(\not p-M_{i}\right) \delta^{i k}+i\left(\gamma_{i k} P_{L}+\gamma_{k i} P_{R}\right) \not p\right] S_{R}^{k j}(p)=-\delta^{i j}} \\
& {\left[\left(\not p-M_{i}\right) \delta^{i k}-i\left(\gamma_{k i}^{*} P_{L}+\gamma_{i k}^{*} P_{R}\right) \not p\right] S_{A}^{k j}(p)=-\delta^{i j} .}
\end{aligned}
$$

The coefficients are given by

$$
\gamma_{i j}=\left(h^{\dagger} h\right)_{i j}\left[\frac{\Theta\left(p^{2}\right) \operatorname{sgn}\left(p_{0}\right)}{16 \pi}\left(1+\frac{2}{e^{\left|p_{0}\right| / T}-1}\right)+i\left(\frac{\ln \frac{\left|p^{2}\right|}{\mu^{2}}}{16 \pi^{2}}+\frac{T^{2}}{6 p^{2}}-\frac{T^{2}}{6 \mu^{2}}\right)\right] \text {. }
$$

In the following, we will first identify the poles and the corresponding residua of the retarded and advanced propagators, and then Fourier transform the solution taking the contribution from the poles into account.

Let us first consider the retarded propagator. In order to find a solution we make a suitable Lorentz decomposition [21, 64, 10],

$$
S_{R}(p)=S_{L L} P_{L}+S_{R R} P_{R}+S_{L R} P_{L \not p}+S_{R L} P_{R} \not p,
$$

where each coefficient is a two-by-two matrix in flavor space. We denote the expression in square brackets on the left-hand side of the Schwinger-Dyson equation (87) by $\Omega$, and perform an analogous decomposition:

$$
\begin{aligned}
& \Omega_{L L}^{i k}=\Omega_{R R}^{i k}=-M_{i} \delta^{i k}, \\
& \Omega_{L R}^{i k}=\delta^{i k}+i \gamma_{i k}, \\
& \Omega_{R L}^{i k}=\delta^{i k}+i \gamma_{k i} .
\end{aligned}
$$


Then the solution of the SD equation reads:

$$
\begin{aligned}
& S_{L L}=-\left[\Omega_{L L}-p^{2} \Omega_{L R} \Omega_{R R}^{-1} \Omega_{R L}\right]^{-1}, \\
& S_{R R}=-\left[\Omega_{R R}-p^{2} \Omega_{R L} \Omega_{L L}^{-1} \Omega_{L R}\right]^{-1}, \\
& S_{L R}=-\Omega_{L L}^{-1} \Omega_{L R} S_{R R}=\left[\Omega_{R R} \Omega_{L R}^{-1} \Omega_{L L}-p^{2} \Omega_{R L}\right]^{-1}, \\
& S_{R L}=-\Omega_{R R}^{-1} \Omega_{R L} S_{L L}=\left[\Omega_{L L} \Omega_{R L}^{-1} \Omega_{R R}-p^{2} \Omega_{L R}\right]^{-1} .
\end{aligned}
$$

The components can be computed explicitly. It is convenient to write them as

$$
S_{X Y}=\frac{s_{X Y}}{H},
$$

where $X, Y \in L, R$, and using the common denominator $H$ given by

$$
\begin{aligned}
H & =M_{1} M_{2} \operatorname{det}\left[\Omega_{R R}-p^{2} \Omega_{R L} \Omega_{L L}^{-1} \Omega_{L R}\right] \\
& =M_{1} M_{2} \operatorname{det}\left[\Omega_{L L}-p^{2} \Omega_{L R} \Omega_{R R}^{-1} \Omega_{R L}\right]=Q^{2} p^{4}+M_{1}^{2} M_{2}^{2} \\
& -p^{2}\left[M_{2}^{2}\left(1+i \gamma_{11}\right)^{2}+M_{1}^{2}\left(1+i \gamma_{22}\right)^{2}-M_{1} M_{2}\left(\gamma_{12}^{2}+\gamma_{21}^{2}\right)\right] \\
Q & =\operatorname{det} \Omega_{L R}=\operatorname{det} \Omega_{R L}=\left(1+i \gamma_{11}\right)\left(1+i \gamma_{22}\right)+\gamma_{12} \gamma_{21}
\end{aligned}
$$

For the enumerators, we obtain

$$
\begin{aligned}
s_{L L} & =\left(\begin{array}{c}
M_{1}\left(M_{2}^{2}-p^{2}\left(1+i \gamma_{22}\right)^{2}\right)+M_{2} p^{2} \gamma_{21}^{2} \\
i p^{2}\left(M_{1} \gamma_{12}\left(1+i \gamma_{22}\right)+M_{2} \gamma_{21}\left(1+i \gamma_{11}\right)\right) \\
i p^{2}\left(M_{1} \gamma_{12}\left(1+i \gamma_{22}\right)+M_{2} \gamma_{21}\left(1+i \gamma_{11}\right)\right) \\
M_{2}\left(M_{1}^{2}-p^{2}\left(1+i \gamma_{11}\right)^{2}\right)+M_{1} p^{2} \gamma_{12}^{2}
\end{array}\right), \\
s_{R R} & =\left.s_{L L}\right|_{\gamma_{12} \mapsto \gamma_{21}}, \\
s_{L R} & =\left(\begin{array}{cc}
M_{2}^{2}\left(1+i \gamma_{11}\right)-p^{2} Q\left(1+i \gamma_{22}\right) & i\left(M_{1} M_{2} \gamma_{12}+p^{2} Q \gamma_{21}\right) \\
i\left(M_{1} M_{2} \gamma_{21}+p^{2} Q \gamma_{12}\right) & M_{1}^{2}\left(1+i \gamma_{22}\right)-p^{2} Q\left(1+i \gamma_{11}\right)
\end{array}\right), \\
s_{R L} & =\left.s_{L R}\right|_{\gamma_{12} \mapsto \gamma_{21}} .
\end{aligned}
$$

The denominator $H$ can be written in the form

$$
H=Q^{2}\left(p^{2}-x_{1}\right)\left(p^{2}-x_{2}\right) .
$$

All the components are proportional to $1 / H$ (note that $H$ is invariant under the replacement $\gamma_{12} \mapsto \gamma_{21}$ ). Therefore, the retarded propagator has poles at $p^{2}=x_{1,2}$. The two complex poles are given by

$$
x_{1,2}=\left.\frac{(V \pm W)^{2}}{4 Q^{2}}\right|_{p^{2}=x_{1,2}},
$$

where the quantities $V$ and $W$ have been defined as

$$
\begin{aligned}
V & =\sqrt{\left(M_{1}\left(1+i \gamma_{22}\right)-M_{2}\left(1+i \gamma_{11}\right)\right)^{2}-M_{1} M_{2}\left(\gamma_{12}+\gamma_{21}\right)^{2}} \\
W & =\sqrt{\left(M_{1}\left(1+i \gamma_{22}\right)+M_{2}\left(1+i \gamma_{11}\right)\right)^{2}-M_{1} M_{2}\left(\gamma_{12}-\gamma_{21}\right)^{2}} .
\end{aligned}
$$

Note that these expressions are implicit equations for the poles, because the coefficients $\gamma_{i j}$ themselves depend on $p^{2}$. It is possible to determine the poles iteratively, starting with an initial guess that we choose as $\gamma_{i j}\left(p^{2}=\mu^{2}\right)$ with 
$\mu=\left(M_{1}+M_{2}\right) / 2$. The poles of the retarded propagator can be parameterized by effective dispersion relations $\omega_{p I}$ and widths $\Gamma_{p I}$,

$$
p_{0}= \pm \omega_{p I}-\frac{i}{2} \Gamma_{p I}, \quad I=1,2,
$$

which are determined by $p^{2}=p_{0}^{2}-\mathbf{p}^{2}=x_{I}$,

$$
\Gamma_{p I}=\frac{\left|\operatorname{Im}\left(x_{I}\right)\right|}{\omega_{p I}}, \quad \omega_{p I}=\sqrt{\operatorname{Re}\left(x_{I}\right)+\Gamma_{p I}^{2} / 4+\mathbf{p}^{2}} .
$$

The pole masses $\left.M_{I}^{\text {pole }} \equiv \omega_{p I}\right|_{\mathbf{p}=0}$ and widths $\left.\Gamma_{I}^{\text {pole }} \equiv \Gamma_{p I}\right|_{\mathbf{p}=0}$ obtained for vanishing spatial momentum are shown in Fig. 3 for a representative choice of parameters. We remind the reader that the masses $M_{1,2}$ are the renormalized mass parameters in the modified $\overline{M S}$ scheme where the mass shift due to the finite temperature is already absorbed into $M_{1,2}$. The relation to the vacuum mass parameters can be easily inferred from the rescaling prescription discussed above.

It is instructive to derive approximate analytical expressions in two limiting cases of interest. Since we are mainly interested in mass splittings of the order of the width, $\left(M_{2}-M_{1}\right) / M_{1} \sim \mathcal{O}\left(\left(h^{\dagger} h\right)_{i j}\right)$, and small Yukawa couplings $\left|\left(h^{\dagger} h\right)_{i j}\right| \ll$ 1 , we will assume in all cases that $\left(M_{2}-M_{1}\right) / M_{1} \gg \max _{i, j}\left|\left(h^{\dagger} h\right)_{i j}\right|^{2}$. We also assume that the different entries are not fine-tuned, such that e.g. the difference $\left(h^{\dagger} h\right)_{22}-\left(h^{\dagger} h\right)_{11}$ is of the same order of magnitude as the individual terms. Provided these assumptions are satisfied, we find that simple approximate expressions can be obtained depending on the relative size of $\left(M_{2}-M_{1}\right) / M_{1}$ and the real part of the flavor off-diagonal combination $\operatorname{Re}\left(h^{\dagger} h\right)_{12} /(8 \pi)$ of Yukawa couplings. Let us first consider the regime $\left(M_{2}-M_{1}\right) / M_{1} \gtrsim \operatorname{Re}\left(h^{\dagger} h\right)_{12} /(8 \pi)$. Here we find that

$$
\begin{aligned}
M_{i}^{\text {pole }} & \simeq M_{i} \\
\Gamma_{i}^{\text {pole }} & \simeq \Gamma_{i} \equiv \frac{\left(h^{\dagger} h\right)_{i i}}{8 \pi} M_{i}\left(1+\frac{2}{e^{M_{i} / T}-1}\right) .
\end{aligned}
$$

Thus, in this case, the pole masses are approximately equal to the renormalized mass parameters, and the widths are given by the same expressions as in the hierarchical case.

If, on the other hand, $\left(M_{2}-M_{1}\right) / M_{1} \lesssim \operatorname{Re}\left(h^{\dagger} h\right)_{12} /(8 \pi)$, we find

$$
\begin{aligned}
M_{i}^{\text {pole }} \simeq & \frac{M_{1}+M_{2}}{2} \pm \frac{\left(M_{2}-M_{1}\right)\left(\left(h^{\dagger} h\right)_{22}-\left(h^{\dagger} h\right)_{11}\right)}{2 \sqrt{\left(\left(h^{\dagger} h\right)_{22}-\left(h^{\dagger} h\right)_{11}\right)^{2}+4\left(\operatorname{Re}\left(h^{\dagger} h\right)_{12}\right)^{2}}} \\
\Gamma_{i}^{\text {pole }} \simeq & \frac{M_{i}}{16 \pi}\left(1+\frac{2}{e^{M_{i} / T}-1}\right)\left(\left(h^{\dagger} h\right)_{11}+\left(h^{\dagger} h\right)_{22}\right. \\
& \left. \pm \sqrt{\left(\left(h^{\dagger} h\right)_{22}-\left(h^{\dagger} h\right)_{11}\right)^{2}+4\left(\operatorname{Re}\left(h^{\dagger} h\right)_{12}\right)^{2}}\right) .
\end{aligned}
$$

Note that the off-diagonal coupling parameter is bounded from above, $\left|\left(h^{\dagger} h\right)_{12}\right|^{2} \leq$ $\left(h^{\dagger} h\right)_{11}\left(h^{\dagger} h\right)_{22}$, such that the effective width cannot become negative. As can be seen in Fig. 3, the transition between the two regimes is relatively fast, i.e. the approximate expressions can be used for most of the considered parameter space. In addition, both cases agree if $\operatorname{Re}\left(h^{\dagger} h\right)_{12} /\left|\left(h^{\dagger} h\right)_{22}-\left(h^{\dagger} h\right)_{11}\right| \ll 1$ is small. We 


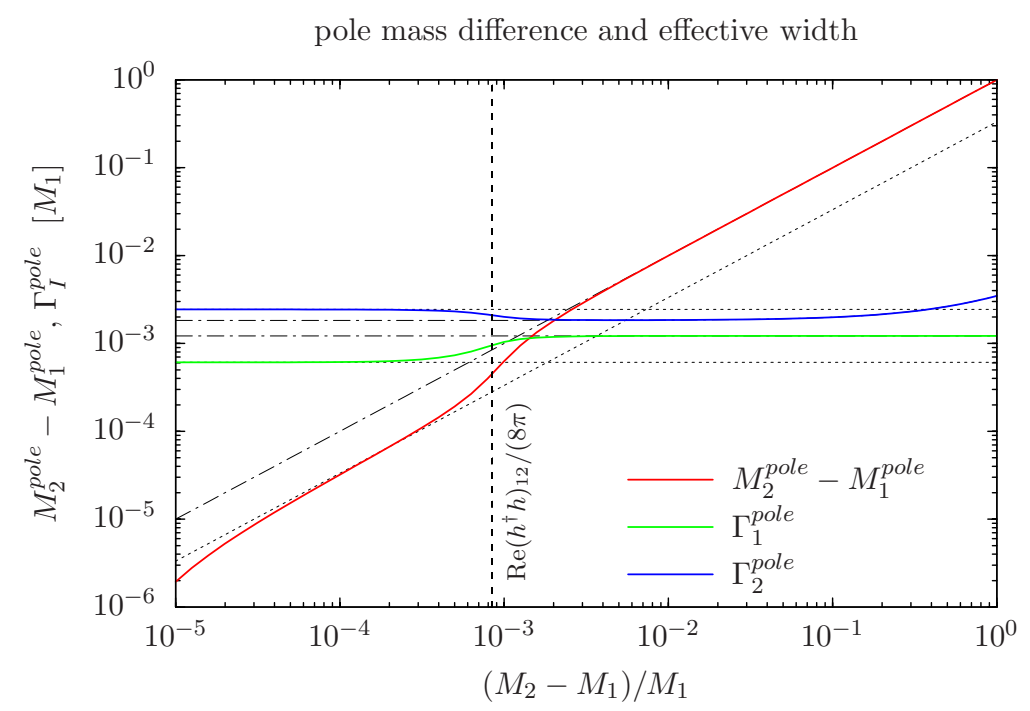

Figure 3: Effective masses $\left.M_{I}^{\text {pole }} \equiv \omega_{p I}\right|_{\mathbf{p}=0}$ and widths $\left.\Gamma_{I}^{\text {pole }} \equiv \Gamma_{p I}\right|_{\mathbf{p}=0}$ of the sterile Majorana neutrinos extracted from the complex poles of the resummed retarded and advanced propagators for $\left(h^{\dagger} h\right)_{11}=0.03,\left(h^{\dagger} h\right)_{22}=0.045$, $\left(h^{\dagger} h\right)_{12}=0.03 \cdot e^{i \pi / 4}$ and $T=0.25 M_{1}$. The black dot-dashed lines show the approximation valid for $\left(M_{2}-M_{1}\right) / M_{1} \gtrsim \operatorname{Re}\left(h^{\dagger} h\right)_{12} /(8 \pi)$, see Eq. (98), and the black dotted lines correspond to the approximate expressions (99) valid for $\operatorname{Re}\left(h^{\dagger} h\right)_{12} /(8 \pi) \gtrsim\left(M_{2}-M_{1}\right) / M_{1} \gg \max _{i, j}\left|\left(h^{\dagger} h\right)_{i j}\right|^{2}$.

find that Eq. (98) yields a reasonable approximation within both regimes if the ratio is smaller than $\sim 1 / 3$.

It turns out that the replacement $\gamma_{i j} \rightarrow \gamma_{i j}\left(p^{2}=\mu^{2}\right)$ yields a rather accurate approximation for small Yukawa couplings and mass splittings of the order or larger than the width. We will use this simplification in the following in order to obtain an analytical solution for the retarded propagator. In addition, we will use the approximation $1+2 /\left(e^{\left|p_{0}\right| / T}-1\right) \approx 1$ for simplicity. The resulting solution can be brought into the form

$$
S_{R}(p)=\frac{Z_{1 R}}{p^{2}-x_{1}}+\frac{Z_{2 R}}{p^{2}-x_{2}},
$$

where the residua $Z_{I R}$ are matrices in flavor space, and can be decomposed analogously to the propagator,

$$
Z_{I R}=Z_{I L L} P_{L}+Z_{I R R} P_{R}+Z_{I L R} P_{L} \not p+Z_{I R L} P_{R} \not p \quad \text { for } I=1,2 .
$$

The components $Z_{I X Y}(X, Y=L, R)$ of the residues can be computed explicitly using

$$
Z_{1 X Y}=\frac{1}{Q^{2}} \frac{s_{X Y}\left(p^{2}=x_{1}\right)}{x_{1}-x_{2}}, \quad Z_{2 X Y}=\frac{1}{Q^{2}} \frac{s_{X Y}\left(p^{2}=x_{2}\right)}{x_{2}-x_{1}} .
$$

The solution for the advanced propagator can be formally obtained by replacing $i \gamma \rightarrow(i \gamma)^{\dagger}$, which implies $\Omega_{X Y} \rightarrow \Omega_{X Y}^{\dagger}$. Using that $\Omega_{L L}$ and $\Omega_{R R}$ are 
hermitian, we find that the advanced propagator is given by

$$
S_{A}(p)=S_{R R}^{\dagger} P_{L}+S_{L L}^{\dagger} P_{R}+S_{L R}^{\dagger} P_{L} \not p+S_{R L}^{\dagger} P_{R} \not p,
$$

where all expressions are evaluated at momentum $p$. For the poles, hermitian conjugation is equivalent to $x_{I} \mapsto x_{I}^{*}$. Therefore, we obtain

$$
S_{A}(p)=\frac{Z_{1 A}}{p^{2}-x_{1}^{*}}+\frac{Z_{2 A}}{p^{2}-x_{2}^{*}}
$$

where $Z_{I A}=Z_{I R R}^{\dagger} P_{L}+Z_{I L L}^{\dagger} P_{R}+Z_{I L R}^{\dagger} P_{L} \not p+Z_{I R L}^{\dagger} P_{R} \not p$ for $I=1,2$.

We are interested in the retarded and advanced propagators in time rather than frequency representation. Both representations are related by the Fourier transformation

$$
S_{R(A) \mathbf{p}}(t)=\int \frac{d p_{0}}{2 \pi} e^{-i p_{0} t} S_{R(A)}(p) .
$$

The Fourier integration can be performed using the Cauchy theorem, and closing the contour in the upper imaginary plane for $t<0$ and in the lower one for $t>0$. Note that, due to the non-analytic behaviour of $\gamma_{i j}$ at the threshold $p^{2}=0$, it is necessary to use a contour which is obtained by dividing a semi-circle into three parts such that the lines corresponding to $\operatorname{Re}\left(p_{0}\right)= \pm|\mathbf{p}|$ are left out. Within the analytical Breit-Wigner approach pursued here, we will take only the contributions from the poles into account, and neglect the contributions that arise from the parts of the contours parallel to the imaginary axis. The latter can lead to deviations from the Breit-Wigner approximation at very late or very early times [65], in which we are not primarily interested here. The approximation will be justified later on by a comparison with numerical solutions. Since the poles of the retarded propagator have a negative imaginary part, the integral vanishes in the case $t<0$. This is precisely what is expected for a retarded propagator. Analogously, the advanced propagator vanishes when $t>0$. We find the result

$$
S_{R(A) \mathbf{p}}(t)= \pm \Theta( \pm t) \sum_{I=1,2} e^{\mp \Gamma_{p I} t / 2}\left[e^{-i \omega_{p I} t} S_{I R(A)}^{-}+e^{+i \omega_{p I} t} S_{I R(A)}^{+}\right]
$$

where the positive and negative frequency coefficients for retarded and advanced function, respectively, are given by

$$
\begin{aligned}
S_{I R}^{ \pm} & = \pm \frac{i}{2} \frac{\left.Z_{I R}\right|_{p^{0}=\mp \omega_{p I}-i \Gamma_{p I} / 2}}{\omega_{p I} \pm i \Gamma_{p I} / 2} \\
S_{I A}^{ \pm} & = \pm \frac{i}{2} \frac{\left.Z_{I A}\right|_{p^{0}=\mp \omega_{p I}+i \Gamma_{p I} / 2}}{\omega_{p I} \mp i \Gamma_{p I} / 2}
\end{aligned}
$$

In the limit of small Yukawa couplings and mass splitting larger than the width, the retarded and advanced propagators approach the well-known result

$$
S_{R(A) \mathbf{p}}^{i j}(t) \rightarrow \pm \Theta( \pm t) e^{\mp \Gamma_{p i} t / 2}\left[\frac{M_{i}-\mathbf{p} \gamma}{\omega_{p i}} \sin \left(\omega_{p i} t\right)+i \gamma_{0} \cos \left(\omega_{p i} t\right)\right] \delta^{i j} .
$$

The flavour off-diagonal contributions, which are suppressed by the Yukawa coupling, are important for the creation of a lepton asymmetry. The leading contributions obtained by expanding in the Yukawa coupling are given in the Appendix. 


\subsection{Lepton asymmetry}

The statistical propagator for the Majorana neutrinos, which describes the nonequilibrium time evolution due to the interaction with the thermal lepton and Higgs background, can be directly obtained by inserting the retarded and advanced propagators into Eq. (75). Next, we obtain the lepton asymmetry generated by the deviation of the Majorana neutrinos from equilibrium from Eq. (76). It is convenient to rewrite it in the form

$$
n_{L}(t)=\int \frac{d^{3} p}{(2 \pi)^{3}} \operatorname{tr}\left[\Delta S_{F \mathbf{p}}^{k l}(0,0) K_{\mathbf{p}}^{l k}(t)-\Delta \bar{S}_{F \mathbf{p}}^{k l}(0,0) \bar{K}_{\mathbf{p}}^{l k}(t)\right] .
$$

The lepton asymmetry depends on the initial conditions for the Majorana neutrino, and on the time-dependent coefficients $K$ which are given by

$$
\begin{aligned}
K_{\mathbf{p}}^{l k}(t) & \equiv i\left(h^{\dagger} h\right)_{j i} \int_{0}^{t} d t^{\prime} \int_{0}^{t} d t^{\prime \prime} \gamma_{0} S_{A \mathbf{p}}^{l j}\left(-t^{\prime \prime}\right) P_{L} S_{\ell \phi \rho \mathbf{p}}\left(t^{\prime \prime}-t^{\prime}\right) P_{R} S_{R \mathbf{p}}^{i k}\left(t^{\prime}\right) \gamma_{0}, \\
\bar{K}_{\mathbf{p}}^{l k}(t) & \equiv i\left(h^{\dagger} h\right)_{i j} \int_{0}^{t} d t^{\prime} \int_{0}^{t} d t^{\prime \prime} \gamma_{0} \bar{S}_{A \mathbf{p}}^{l j}\left(-t^{\prime \prime}\right) P_{L} S_{\ell \phi \rho \mathbf{p}}\left(t^{\prime \prime}-t^{\prime}\right) P_{R} \bar{S}_{R \mathbf{p}}^{i k}\left(t^{\prime}\right) \gamma_{0} .
\end{aligned}
$$

In order to compute the asymmetry, we insert Eq. (80) for the lepton-Higgs loop $S_{\ell \phi \rho \mathbf{p}}\left(t^{\prime \prime}-t^{\prime}\right)$. Using the result for the retarded and advanced propagators, we can now decompose the double-time integral,

$$
\begin{aligned}
K_{\mathbf{p}}^{l k}(t) & =\int \frac{d^{3} q}{(2 \pi)^{3} 2 q} \sum_{I, J=1,2} \sum_{\epsilon_{k}= \pm 1} \gamma_{J I}^{l k}\left(\left\{\epsilon_{k}\right\}\right) L_{I J}\left(t,\left\{\epsilon_{k}\right\}\right), \\
\bar{K}_{\mathbf{p}}^{l k}(t) & =\int \frac{d^{3} q}{(2 \pi)^{3} 2 q} \sum_{I, J=1,2} \sum_{\epsilon_{k}= \pm 1} \bar{\gamma}_{J I}^{l k}\left(\left\{\epsilon_{k}\right\}\right) L_{I J}\left(t,\left\{\epsilon_{k}\right\}\right),
\end{aligned}
$$

where all the $\epsilon_{k}$ are summed over + and - modes. The coefficients are given by

$$
\begin{aligned}
& \gamma_{J I}^{l k}\left(\left\{\epsilon_{k}\right\}\right)=-i\left(h^{\dagger} h\right)_{j i} \gamma_{0} S_{A J}^{l j \epsilon_{1}} P_{L} S_{\ell \phi} \epsilon_{\rho \mathbf{p}} \epsilon_{3} P_{R} S_{R I}^{i k \epsilon_{4}} \gamma_{0} \\
& \bar{\gamma}_{J I}^{l k}\left(\left\{\epsilon_{k}\right\}\right)=-i\left(h^{\dagger} h\right)_{i j} \gamma_{0} \bar{S}_{A J}^{l j \epsilon_{1}} P_{L} S_{\ell \phi} \epsilon_{\rho \mathbf{p}} \epsilon_{3} P_{R} \bar{S}_{R I}^{i k \epsilon_{4}} \gamma_{0}
\end{aligned}
$$

and the time integrals are explicitly given by

$$
L_{I J}\left(t,\left\{\epsilon_{k}\right\}\right)=\int_{0}^{t} d t^{\prime} \int_{0}^{t} d t^{\prime \prime} e^{i A t^{\prime \prime}} e^{-i B t^{\prime}} e^{-\Gamma_{\ell \phi}\left|t^{\prime \prime}-t^{\prime}\right| / 2}
$$

where

$$
\begin{aligned}
& A=\epsilon_{2} k+\epsilon_{3} q-\epsilon_{1} \omega_{p J}+i \Gamma_{p J} / 2, \\
& B=\epsilon_{2} k+\epsilon_{3} q-\epsilon_{4} \omega_{p I}-i \Gamma_{p I} / 2 .
\end{aligned}
$$

The double time integral can be easily performed now. Since the interesting dynamics occurs at time scales of $t \sim 1 / \Gamma_{p I}$, and the widths of leptons and the Higgs are much larger than the one of the Majorana neutrinos, it is reasonable to consider the limit where

$$
\Gamma_{\ell \phi} \cdot t \gg 1
$$


In this limit,

$$
\begin{aligned}
L_{I J}\left(t,\left\{\epsilon_{k}\right\}\right) \simeq & \frac{i \Gamma_{\ell \phi}}{2(A-B)}\left(\frac{1}{A^{2}+\Gamma_{\ell \phi}^{2} / 4}+\frac{1}{B^{2}+\Gamma_{\ell \phi}^{2} / 4}\right)\left[1-e^{i(A-B) t}\right] \\
& +\frac{A B-\Gamma_{\ell \phi}^{2} / 4}{\left(A^{2}+\Gamma_{\ell \phi}^{2} / 4\right)\left(B^{2}+\Gamma_{\ell \phi}^{2} / 4\right)}\left[1+e^{i(A-B) t}\right] .
\end{aligned}
$$

Lets identify which terms yield the leading contribution to the lepton asymmetry. Formally, we have to look for contributions which can be of order $1 / \Gamma_{1}$, $1 / \Gamma_{2}$ or $1 /\left(M_{1}-M_{2}\right)$. Only the term proportional to $1 /(A-B)$ can be of that order, provided that $\epsilon_{1}=\epsilon_{4}$. Then for $I=J$ we get a contribution of order $1 / \Gamma_{I}$, and for $I \neq J$ of order $1 /\left(\omega_{p 1}-\omega_{p 2}+i\left(\Gamma_{p 1}+\Gamma_{p 2}\right) / 2\right)$. The latter will be important in the maximal resonant case. Thus, from now on we only take the $1 /(A-B)$ term into account.

Furthermore, the terms involving $A^{2}$ or $B^{2}$ are unsuppressed only if the corresponding real parts of $\mathrm{A}$ or $\mathrm{B}$ can become zero within the phase-space integration. This is only the case for $\epsilon_{2}=\epsilon_{3}=\epsilon_{1}$ or $\epsilon_{2}=\epsilon_{3}=\epsilon_{4}$, respectively. Altogether, this means the leading contribution arises when all $\epsilon$ 's are identical,

$$
\left.L_{I J}\left(t,\left\{\epsilon_{k}\right\}\right) \rightarrow L_{I J}^{\epsilon}(t) \equiv L_{I J}\left(t,\left\{\epsilon_{k}\right\}\right)\right|_{\epsilon_{1}=\epsilon_{2}=\epsilon_{3}=\epsilon_{4} \equiv \epsilon} .
$$

Then, we find the following result:

$$
\begin{aligned}
& L_{I J}^{\epsilon}(t)=\frac{i \epsilon}{\omega_{p I}-\omega_{p J}+i \epsilon\left(\Gamma_{p I}+\Gamma_{p J}\right) / 2}\left[1-e^{i \epsilon\left(\omega_{p I}-\omega_{p J}\right) t-\left(\Gamma_{p I}+\Gamma_{p J}\right) t / 2}\right] \\
& \times\left(\frac{\Gamma_{\ell \phi} / 2}{\left(\omega_{p J}-k-q+i \epsilon \Gamma_{p J} / 2\right)^{2}+\Gamma_{\ell \phi}^{2} / 4}+\frac{\Gamma_{\ell \phi} / 2}{\left(\omega_{p I}-k-q-i \epsilon \Gamma_{p I} / 2\right)^{2}+\Gamma_{\ell \phi}^{2} / 4}\right) .
\end{aligned}
$$

The entries on the diagonal can be written in a simpler form. They are realvalued and equal for both signs of $\epsilon, L_{I I}^{+}(t)=L_{I I}^{-}(t) \equiv L_{I I}(t)$, with

$$
L_{I I}(t)=\frac{1-e^{-\Gamma_{p I} t}}{\Gamma_{p I}} \operatorname{Re}\left(\frac{\Gamma_{\ell \phi}}{\left(\omega_{p I}-k-q+i \Gamma_{p I} / 2\right)^{2}+\Gamma_{\ell \phi}^{2} / 4}\right) .
$$

Note that the time-dependence precisely coincides with the time-evolution of the lepton asymmetry that would be expected from a Boltzmann approach.

The off-diagonal entries, i.e. for $I \neq J$, lead to an oscillatory behaviour and will be important in the maximal resonant regime. They fulfill the relations

$$
L_{12}^{ \pm}(t)=L_{21}^{ \pm}(t)^{*}=L_{12}^{\mp}(t)^{*} .
$$

Note that all entries except $L_{11}$ become suppressed in the hierarchical limit $M_{2} \sim \omega_{p 2} \gg M_{1}$. On the other hand, in the extreme degenerate limit $M_{2} \rightarrow M_{1}$, $\Gamma_{2} \rightarrow \Gamma_{1}$, all the entries $L_{I J}$ become equal.

Putting the results together, we obtain the following expression for the lepton asymmetry:

$$
n_{L}(t)=\int \frac{d^{3} p}{(2 \pi)^{3}} \int \frac{d^{3} q}{(2 \pi)^{3} 2 q} \sum_{I, J=1,2} \sum_{\epsilon= \pm 1} F_{J I}^{\epsilon} L_{I J}^{\epsilon}(t),
$$


where

$$
F_{J I}^{\epsilon}=\operatorname{tr}\left[\Delta S_{F}^{k l} \mathbf{p}(0,0) \gamma_{J I}^{l k \epsilon}-\Delta \bar{S}_{F \mathbf{p}}^{k l}(0,0) \bar{\gamma}_{J I}^{l k \epsilon}\right],
$$

and

$$
\begin{aligned}
& \gamma_{J I}^{l k \epsilon}=\gamma_{J I}^{l k}\left(\left\{\epsilon_{k}=\epsilon\right\}\right)=-i\left(h^{\dagger} h\right)_{j i} \gamma_{0} S_{A J}^{l j \epsilon} P_{L} S_{\ell \phi}^{\epsilon \epsilon} P_{\mathbf{p}} P_{R} S_{R I}^{i k \epsilon} \gamma_{0} \\
& \bar{\gamma}_{J I}^{l k \epsilon}=\bar{\gamma}_{J I}^{l k}\left(\left\{\epsilon_{k}=\epsilon\right\}\right)=-i\left(h^{\dagger} h\right)_{i j} \gamma_{0} \bar{S}_{A J}^{l j \epsilon} P_{L} S_{\ell \phi}^{\epsilon \epsilon} P_{\mathbf{p}} P_{R} \bar{S}_{R I}^{i k \epsilon} \gamma_{0} .
\end{aligned}
$$

Using the properties of $L_{I J}^{\epsilon}(t)$, one can write the lepton asymmetry as a sum of Boltzmann-like and oscillatory contributions,

$$
\begin{aligned}
n_{L}(t)= & \int \frac{d^{3} p}{(2 \pi)^{3}} \int \frac{d^{3} q}{(2 \pi)^{3} 2 q}\left[F_{11} L_{11}(t)+F_{22} L_{22}(t)\right. \\
& \left.+F_{12}^{s} \operatorname{Re}\left(L_{21}^{+}(t)\right)+F_{12}^{a} \operatorname{Im}\left(L_{21}^{+}(t)\right)\right],
\end{aligned}
$$

where the coefficients $F$ contain all the information about the interaction, the $C P$ violation and the initial state,

$$
\begin{aligned}
& F_{I I} \equiv F_{I I}^{+}+F_{I I}^{-}, \\
& F_{12}^{s} \equiv F_{12}^{+}+F_{12}^{-}+F_{21}^{+}+F_{21}^{-}, \\
& F_{12}^{a} \equiv i\left(F_{12}^{+}-F_{12}^{-}-F_{21}^{+}+F_{21}^{-}\right) .
\end{aligned}
$$

and the functions $L(t)$ characterize the time-evolution,

$$
\begin{aligned}
L_{I I}(t)= & \frac{1-e^{-\Gamma_{p I} t}}{\Gamma_{p I}} \operatorname{Re}\left(\frac{\Gamma_{\ell \phi}}{\left(\omega_{p I}-k-q+i \Gamma_{p I} / 2\right)^{2}+\Gamma_{\ell \phi}^{2} / 4}\right) \\
L_{21}^{+}(t)= & \frac{1-e^{-i\left(\omega_{p 1}-\omega_{p 2}\right) t} e^{-\Gamma_{p} t}}{\Gamma_{p}+i\left(\omega_{p 1}-\omega_{p 2}\right)}\left(\frac{\Gamma_{\ell \phi} / 2}{\left(\omega_{p 1}-k-q+i \Gamma_{p 1} / 2\right)^{2}+\Gamma_{\ell \phi}^{2} / 4}\right. \\
& \left.+\frac{\Gamma_{\ell \phi} / 2}{\left(\omega_{p 2}-k-q-i \Gamma_{p 2} / 2\right)^{2}+\Gamma_{\ell \phi}^{2} / 4}\right) .
\end{aligned}
$$

where $\Gamma_{p} \equiv\left(\Gamma_{p 1}+\Gamma_{p 2}\right) / 2$.

One non-trivial element that remains to be specified is the initial condition for the statistical neutrino propagator $\Delta S_{F \mathbf{p}}(0,0)$. In principle, we have a great freedom to impose initial conditions, as far as the validity of the approach is concerned. Clearly, it is desirable to motivate the choice by some physical considerations. Concretely, we will assume that the neutrinos are in 'vacuum' at $t=t^{\prime}=0$, i.e.

$$
\left.S_{F \mathbf{p}}\left(t, t^{\prime}\right)\right|_{t=t^{\prime}=0}=\left.S_{F \mathbf{p}}^{v a c}\left(t-t^{\prime}\right)\right|_{t=t^{\prime}=0} .
$$

The Majorana neutrinos will then be produced by the Yukawa interactions with the thermal bath of the Higgs boson and leptons. This resembles a typical initial condition often used for leptogenesis calculations.

By definition, the above choice implies that the deviation from equilibrium at the initial time is given by

$$
\left.\Delta S_{F \mathbf{p}}\left(t, t^{\prime}\right)\right|_{t=t^{\prime}=0}=\left.S_{F \mathbf{p}}^{v a c}\left(t-t^{\prime}\right)\right|_{t=t^{\prime}=0}-\left.S_{F \mathbf{p}}^{t h}\left(t-t^{\prime}\right)\right|_{t=t^{\prime}=0} .
$$




\subsection{Expansion in the Yukawa couplings}

By combining the results for the lepton asymmetry (119) and the coefficients (106) of the positive and negative frequency modes of the retarded and advanced propagators, it is possible to determine the resonantly generated asymmetry for a given set of Yukawa couplings $\left(h^{\dagger} h\right)_{i j}$ and mass parameters $M_{1,2}$. The approximations done so far allow us to choose a mass difference of the order of the width, $\left(M_{2}-M_{1}\right) / M_{1} \sim \mathcal{O}\left(\left(h^{\dagger} h\right)_{i j}\right) /(8 \pi) \ll 1$, which is the regime in which we expect a maximal enhancement. It is possible to simplify the analytical expressions considerably within the parameter region $\operatorname{Re}\left(h^{\dagger} h\right)_{12} \ll$ $\left|\left(h^{\dagger} h\right)_{22}-\left(h^{\dagger} h\right)_{11}\right|$ and $\left(M_{2}-M_{1}\right) / M_{1} \gtrsim \operatorname{Re}\left(h^{\dagger} h\right)_{12} /(8 \pi)$. We will therefore first discuss the analytical solution in this regime, and then compare it to the full analytical solution in Breit-Wigner approximation as well as a full numerical solution.

In the parameter region described above, the pole masses are approximately equal to the renormalized mass parameters $M_{1,2}$ and the widths are equal to the usual expression for the decay rate $\Gamma_{1,2}$ given in Eq. (98). The dispersion relation and damping rate of the retarded and advanced propagators are given by $\omega_{p I} \simeq \sqrt{M_{I}^{2}+\mathbf{p}^{2}}$ and $\Gamma_{p I} \simeq \Gamma_{I} M_{I} / \omega_{p I}$. Then it is possible to obtain explicit analytical expressions for the coefficients $F_{I J}$ defined in Eq. (117) by systematically expanding the solutions (93) for retarded and advanced propagators for small Yukawa couplings $\left(h^{\dagger} h\right)_{i j} \ll 1$. In particular, at leading order in the Yukawa couplings, the vacuum initial condition is given by

$$
\left.\Delta S_{F \mathbf{p}}^{i j}\left(t, t^{\prime}\right)\right|_{t=t^{\prime}=0} \simeq \delta^{i j} f_{F D}\left(\omega_{p i}\right) \frac{M_{i}-\mathbf{p} \gamma}{\omega_{p i}} .
$$

The result for the coefficients $F_{I J}$ is given in the Appendix, including also several intermediate steps of the calculation. The result obtained for the lepton asymmetry will be discussed in the following section.

\section{$5 \quad$ Result for the lepton asymmetry}

In this section, we will first compare our analytical result for the lepton asymmetry obtained within the Kadanoff-Baym approach in Breit-Wigner approximation with the one obtained from a Boltzmann treatment. Then, we will compare various analytical, semi-analytical and numerical results obtained within the Kadanoff-Baym framework.

\subsection{Comparison of Kadanoff-Baym and Boltzmann}

Let us start with discussing the explicit analytical result in Breit-Wigner approximation, and compare it to the well-known Boltzmann approach. More specifically, we will first present the result that is obtained under the assumptions described in section 4.3. By inserting the coefficients $F_{I J}$ obtained at leading order in the Yukawa couplings into the expression (119) for the lepton 
asymmetry, we obtain for the time-evolution of the total lepton number:

$$
\begin{aligned}
n_{L}(t)= & \frac{\operatorname{Im}\left[\left(h^{\dagger} h\right)_{12}^{2}\right]}{8 \pi} \int \frac{d^{3} p}{(2 \pi)^{3}} \int \frac{d^{3} q}{(2 \pi)^{3} 2 q} \int \frac{d^{3} k}{(2 \pi)^{3} 2 k}(2 \pi)^{3} \delta(\mathbf{p}-\mathbf{k}-\mathbf{q}) \\
& \times\left(1+f_{\phi}(q)-f_{\ell}(k)\right) \\
& \times\left[\sum_{I=1,2} \frac{4 k \cdot p_{I}}{\omega_{p I}} f_{F D}\left(\omega_{p I}\right) \operatorname{Re}\left(\frac{M_{1} M_{2} L_{I I}(t)}{M_{2}^{2}-M_{1}^{2}-i M_{1} \Gamma_{1}+i M_{2} \Gamma_{2}}\right)\right. \\
& -\frac{4 k \cdot p_{2}}{\omega_{p}} f_{F D}\left(\omega_{p 1}\right) \operatorname{Re}\left(\frac{M_{1} M_{2} L_{21}^{+}(t)}{M_{2}^{2}-M_{1}^{2}-i M_{1} \Gamma_{1}+i M_{2} \Gamma_{2}}\right) \\
& \left.-\frac{4 k \cdot p_{1}}{\omega_{p}} f_{F D}\left(\omega_{p 2}\right) \operatorname{Re}\left(\frac{M_{1} M_{2} L_{21}^{+}(t)^{*}}{M_{2}^{2}-M_{1}^{2}-i M_{1} \Gamma_{1}+i M_{2} \Gamma_{2}}\right)\right],
\end{aligned}
$$

where $q=|\mathbf{q}|$ and $k=|\mathbf{k}|$ denote the Higgs and lepton energies, $\omega_{p I}=$ $\sqrt{M_{I}^{2}+\mathbf{p}^{2}}$ are the energies of the Majorana neutrinos $(I=1,2)$, and $k \cdot p_{I} \equiv$ $k \omega_{p I}-\mathbf{k} p$. We have also defined $\omega_{p} \equiv 2 \omega_{p 1} \omega_{p 2} /\left(\omega_{p 1}+\omega_{p 2}\right)$. The time-dependence is described by the functions $L(t)$. Neglecting Yukawa-suppressed contributions in the expressions (121) yields

$$
\begin{aligned}
L_{I I}(t)= & \frac{1-e^{-\Gamma_{p I} t}}{\Gamma_{p I}} \frac{\Gamma_{\ell \phi}}{\left(\omega_{p I}-k-q\right)^{2}+\Gamma_{\ell \phi}^{2} / 4}, \\
L_{21}^{+}(t)= & \frac{1-e^{-i\left(\omega_{p 1}-\omega_{p 2}\right) t} e^{-\Gamma_{p} t}}{\Gamma_{p}+i\left(\omega_{p 1}-\omega_{p 2}\right)}\left(\frac{\Gamma_{\ell \phi} / 2}{\left(\omega_{p 1}-k-q\right)^{2}+\Gamma_{\ell \phi}^{2} / 4}\right. \\
& \left.+\frac{\Gamma_{\ell \phi} / 2}{\left(\omega_{p 2}-k-q\right)^{2}+\Gamma_{\ell \phi}^{2} / 4}\right),
\end{aligned}
$$

where $\Gamma_{\ell \phi}=\Gamma_{\ell}+\Gamma_{\phi}$ is the sum of the thermal width of lepton and Higgs, and $\Gamma_{p I}=M_{I} \Gamma_{I} / \omega_{p I}$ is related to the decay widths of the Majorana neutrinos. Furthermore, we have defined $\Gamma_{p} \equiv\left(\Gamma_{p 1}+\Gamma_{p 2}\right) / 2$.

The flavor-diagonal contributions $L_{11}(t)$ and $L_{22}(t)$ feature a time-dependence that is also expected within the Boltzmann approach for the contribution from the neutrino species $N_{1}$ and $N_{2}$, respectively. In addition, there exists a flavor off-diagonal contribution $L_{21}^{+}(t)$, which has an oscillating behaviour with frequency given by the energy difference $\omega_{p 1}-\omega_{p 2}$ of the two Majorana neutrino species. The latter can be interpreted as the contribution from coherent transitions between the neutrino mass eigenstates $N_{1}$ and $N_{2}$ due to the off-diagonal Yukawa coupling $\left(h^{\dagger} h\right)_{12}$.

It is instructive to compare the result for the lepton asymmetry with the classical Boltzmann approach. Within the latter, the lepton asymmetry is determined by the Boltzmann equation

$$
\begin{aligned}
\frac{d n_{L}^{\text {Boltzmann }}}{d t}= & \sum_{I=1,2} \int \frac{d^{3} p}{(2 \pi)^{3} \omega_{p I}} \int \frac{d^{3} q}{(2 \pi)^{3} 2 q} \int \frac{d^{3} k}{(2 \pi)^{3} 2 k}(2 \pi)^{4} \delta\left(p_{I}-k-q\right) \\
& \times \epsilon_{I}^{C P}\left|\mathcal{M}_{N_{I} \rightarrow \ell \phi}\right|^{2}\left[f_{N_{I}}\left(1+f_{\phi}\right)\left(1-f_{\ell}\right)-\left(1-f_{N_{I}}\right) f_{\phi} f_{\ell}\right]
\end{aligned}
$$

where

$$
\epsilon_{I}^{C P}=\frac{1}{\left(h^{\dagger} h\right)_{I I}} \times \frac{\operatorname{Im}\left[\left(h^{\dagger} h\right)_{I J}^{2}\right]}{8 \pi} \frac{M_{I} M_{J}\left(M_{J}^{2}-M_{I}^{2}\right)}{\left(M_{J}^{2}-M_{I}^{2}\right)^{2}+\left(M_{I} \Gamma_{I}-M_{J} \Gamma_{J}\right)^{2}},
$$


is the 'wave' contribution to the $C P$-violating parameter [22] (see also [10]), and $\left|\mathcal{M}_{N_{I} \rightarrow \ell \phi}\right|^{2}=4 k \cdot p_{I}\left(h^{\dagger} h\right)_{I I}$ is related to the tree-level matrix element for the decay $N_{I} \rightarrow \ell \phi$. For the setup considered here, the time-evolution of the classical distribution function is simply given by $f_{N_{I}}(t)=f_{F D}\left(\omega_{p I}\right)\left(1-e^{-\Gamma_{p I} t}\right)$.

In the following, we will compare the Kadanoff-Baym and the Boltzmann results for a hierarchical and a quasidegenerate Majorana neutrino mass spectrum.

\section{Hierarchical limit}

In the hierarchical limit $M_{2} \gg M_{1}$, the contributions proportional to the FermiDirac distribution evaluated at the energy of $N_{2}, f_{F D}\left(\omega_{p 2}\right)$, are exponentially suppressed for the relevant temperature range $T \sim M_{1}$. In addition, the coherent contribution $L_{21}^{+}(t) \propto 1 /\left(\Gamma_{p}+i\left(\omega_{p 1}-\omega_{p 2}\right)\right) \sim i / \omega_{p 2}$ is strongly suppressed compared to the flavor-diagonal contribution $L_{11}(t) \propto 1 / \Gamma_{p 1}$. The lepton asymmetry obtained in the Kadanoff-Baym approach is thus given by

$$
\begin{aligned}
n_{L}(t)= & \frac{\operatorname{Im}\left[\left(h^{\dagger} h\right)_{12}^{2}\right]}{8 \pi} \frac{M_{1}}{M_{2}} \int \frac{d^{3} p}{(2 \pi)^{3} \omega_{p 1}} \int \frac{d^{3} q}{(2 \pi)^{3} 2 q} \int \frac{d^{3} k}{(2 \pi)^{3} 2 k} \\
& \times(2 \pi)^{3} \delta(\mathbf{p}-\mathbf{k}-\mathbf{q}) \times \frac{\Gamma_{\ell \phi}}{\left(\omega_{p 1}-k-q\right)^{2}+\Gamma_{\ell \phi}^{2} / 4} \\
& \times 4 k \cdot p_{1}\left(1+f_{\phi}(q)-f_{\ell}(k)\right) f_{F D}\left(\omega_{p 1}\right) \frac{1-e^{-\Gamma_{p 1} t}}{\Gamma_{p 1}} .
\end{aligned}
$$

For comparison, within the classical Boltzmann approach one finds that

$$
\begin{aligned}
n_{L}^{\text {Boltzmann }}(t)= & \frac{\operatorname{Im}\left[\left(h^{\dagger} h\right)_{12}^{2}\right]}{8 \pi} \frac{M_{1}}{M_{2}} \int \frac{d^{3} p}{(2 \pi)^{3} \omega_{p 1}} \int \frac{d^{3} q}{(2 \pi)^{3} 2 q} \int \frac{d^{3} k}{(2 \pi)^{3} 2 k} \\
& \times(2 \pi)^{3} \delta(\mathbf{p}-\mathbf{k}-\mathbf{q}) \times 2 \pi \delta\left(\omega_{p 1}-k-q\right) \\
& \times 4 k \cdot p_{1}\left(1+f_{\phi}(q)-f_{\ell}(k)\right) f_{F D}\left(\omega_{p 1}\right) \frac{1-e^{-\Gamma_{p 1} t}}{\Gamma_{p 1}} .
\end{aligned}
$$

Thus, the thermal width of lepton and Higgs $\Gamma_{\ell \phi}=\Gamma_{\ell}+\Gamma_{\phi}$ leads to a replacement of the on-shell delta function in the Boltzmann equations by a BreitWigner curve, in accordance with [37],

$$
2 \pi \delta\left(\omega_{p}-k-q\right) \rightarrow \frac{\Gamma_{\ell \phi}}{\left(\omega_{p}-k-q\right)^{2}+\Gamma_{\ell \phi}^{2} / 4} .
$$

\section{Degenerate limit}

In the limit where the masses are quasi-degenerate, $\left|M_{1}-M_{2}\right| \ll M_{1,2}$, we approximate $\omega_{p 1} \approx \omega_{p 2} \approx \omega_{p}$ in all terms except for those containing the difference 
of the energies. Then we obtain

$$
\begin{aligned}
n_{L}(t)= & \frac{\operatorname{Im}\left[\left(h^{\dagger} h\right)_{12}^{2}\right]}{8 \pi} \frac{M_{1} M_{2}\left(M_{2}^{2}-M_{1}^{2}\right)}{\left(M_{2}^{2}-M_{1}^{2}\right)^{2}+\left(M_{1} \Gamma_{1}-M_{2} \Gamma_{2}\right)^{2}} \\
& \times \int \frac{d^{3} p}{(2 \pi)^{3} \omega_{p}} \int \frac{d^{3} q}{(2 \pi)^{3} 2 q} \int \frac{d^{3} k}{(2 \pi)^{3} 2 k} \\
& \times(2 \pi)^{3} \delta(\mathbf{p}-\mathbf{k}-\mathbf{q}) \times \frac{\Gamma_{\ell \phi}}{\left(\omega_{p}-k-q\right)^{2}+\Gamma_{\ell \phi}^{2} / 4} \\
& \times 4 k \cdot p\left(1+f_{\phi}(q)-f_{\ell}(k)\right) f_{F D}\left(\omega_{p}\right) \\
& \times\left[\sum_{I=1,2} \frac{1-e^{-\Gamma_{p I} t}}{\Gamma_{p I}}-4 \operatorname{Re}\left(\frac{1-e^{-i\left(\omega_{p 1}-\omega_{p 2}\right) t} e^{-\left(\Gamma_{p 1}+\Gamma_{p 2}\right) t / 2}}{\Gamma_{p 1}+\Gamma_{p 2}+2 i\left(\omega_{p 1}-\omega_{p 2}\right)}\right)\right] .
\end{aligned}
$$

For comparison, the Boltzmann result in the degenerate limit reads

$$
\begin{aligned}
n_{L}^{\text {Boltzmann }}(t)= & \frac{\operatorname{Im}\left[\left(h^{\dagger} h\right)_{12}^{2}\right]}{8 \pi} \frac{M_{1} M_{2}\left(M_{2}^{2}-M_{1}^{2}\right)}{\left(M_{2}^{2}-M_{1}^{2}\right)^{2}+\left(M_{1} \Gamma_{1}-M_{2} \Gamma_{2}\right)^{2}} \\
& \times \int \frac{d^{3} p}{(2 \pi)^{3} \omega_{p 1}} \int \frac{d^{3} q}{(2 \pi)^{3} 2 q} \int \frac{d^{3} k}{(2 \pi)^{3} 2 k} \\
& \times(2 \pi)^{3} \delta(\mathbf{p}-\mathbf{k}-\mathbf{q}) \times 2 \pi \delta\left(\omega_{p}-k-q\right) \\
& \times 4 k \cdot p\left(1+f_{\phi}(q)-f_{\ell}(k)\right) f_{F D}\left(\omega_{p}\right)\left[\sum_{I=1,2} \frac{1-e^{-\Gamma_{p I} t}}{\Gamma_{p I}}\right] .
\end{aligned}
$$

The first line of both results agrees, and corresponds to the resonant enhancement described by the 'usual' $C P$-violating parameter $\epsilon_{i}^{C P}$. Note in particular that in the denominator the 'regulator' $M_{1} \Gamma_{1}-M_{2} \Gamma_{2}$ appears, in accordance with [22]. Furthermore, as in the hierarchical case, the on-shell delta function for the energies in the Boltzmann result is replaced by a Breit-Wigner curve in the Kadanoff-Baym result.

Finally, the Kadanoff-Baym result features an additional contribution inside the square brackets in the last line as compared to the Boltzmann result. It results from the flavor off-diagonal contribution $L_{21}^{+}(t)$. As discussed above, it can be attributed to the contribution to the lepton asymmetry from coherent flavor transitions between the Majorana neutrino species. It becomes comparable in size with the Boltzmann-like contributions when the energy difference $\omega_{p 1}-\omega_{p 2}$ is of the order of the average of the decay rates, $\left(\Gamma_{p 1}+\Gamma_{p 2}\right) / 2$, of the Majorana neutrinos. This case occurs when the mass difference $\Delta M=M_{2}-M_{1}$ of the Majorana neutrino mass eigenstates is of the order of the widths $\Gamma_{I}$. Such a situation has been frequently considered, because it leads to the maximal possible resonant enhancement of the $C P$ asymmetry.

The time-evolution of the lepton asymmetry $n_{L}(t, \mathbf{p})$, defined as $n_{L}(t) \equiv$ $\int d^{3} p /(2 \pi)^{3} n_{L}(t, \mathbf{p})$, is shown in Fig. 4 for various choices of $\Delta M_{21}^{2} \equiv M_{2}^{2}-$ $M_{1}^{2}$. When the splitting is very large compared to the product $M_{I} \Gamma_{I}(I=$ $1,2)$, the Kadanoff-Baym solution oscillates around the Boltzmann result, and the value at large times is very similar for both cases. When the splitting is of comparable size as the product of the widths and the masses, there are significant differences. Finally, when $M_{2}^{2}-M_{1}^{2} \lesssim\left|M_{2} \Gamma_{2}-M_{1} \Gamma_{1}\right|$, the lepton asymmetry obtained in the Kadanoff-Baym analysis is suppressed compared to 


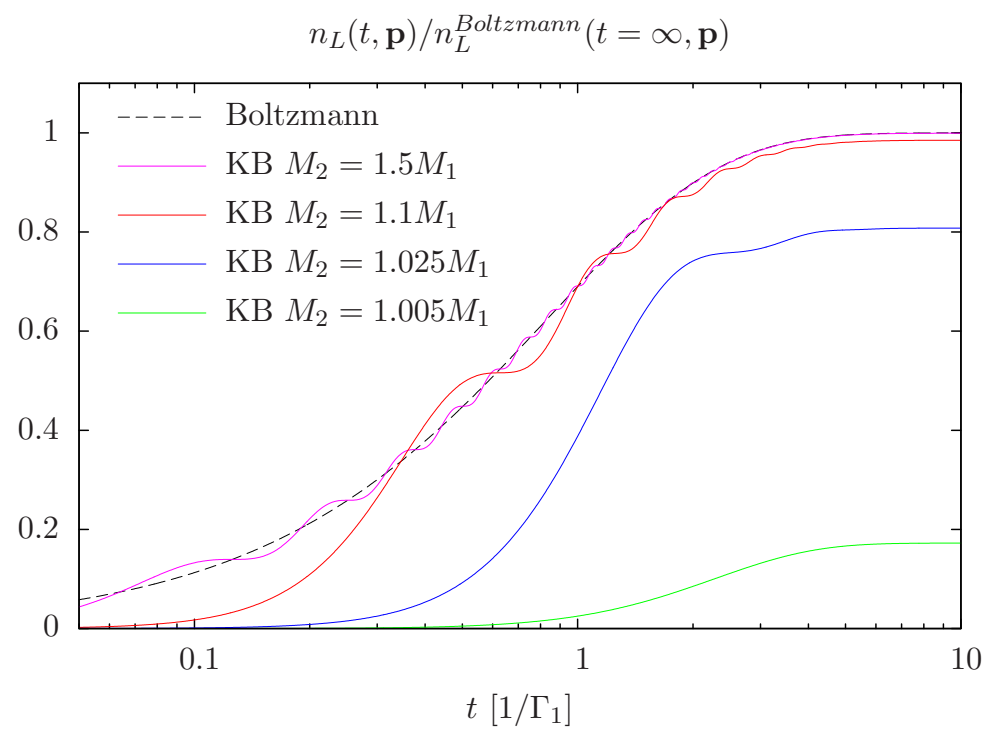

Figure 4: Time-evolution of the lepton asymmetry $n_{L}(t, \mathbf{p})$. Shown is the Boltzmann result (dashed) and the result obtained from the Kadanoff-Baym equations, for various values of the mass-squared splitting $M_{2}^{2}-M_{1}^{2}$ (solid lines). All curves are normalized such that the Boltzmann result approaches unity. For the widths we have chosen $\Gamma_{1}=0.01 M_{1}, \Gamma_{2}=0.015 M_{1}$, and $\mathbf{p}=0$, for illustration.

the one predicted by the Boltzmann approach. The reason is that the coherent contribution has the tendency to cancel part of the Boltzmann-like contributions to the lepton asymmetry. Note that to obtain Fig. 4 we have neglected the effect of the thermal width of lepton and Higgs. Their inclusion would multiply the Kadanoff-Baym results for $n_{L}(t)$ by an additional overall factor, that is identical to the ratio of lepton asymmetries obtained from Kadanoff-Baym and Boltzmann analyses in the hierarchical limit.

In order to quantify the suppression of the lepton asymmetry that results from the additional coherent contributions, it is useful to investigate the parametric dependence on the mass-splitting, and to identify under which conditions the maximal possible resonant enhancement occurs. Within the Boltzmann approach, the resonant enhancement of the lepton asymmetry is maximized provided that the function

$$
R^{\text {Boltzmann }} \equiv \frac{M_{1} M_{2}\left(M_{2}^{2}-M_{1}^{2}\right)}{\left(M_{2}^{2}-M_{1}^{2}\right)^{2}+\left(M_{1} \Gamma_{1}-M_{2} \Gamma_{2}\right)^{2}},
$$

is maximally large. We may assume $M_{2}>M_{1}$ without loss of generality. The maximum of the function $R^{\text {Boltzmann }}$ occurs when $M_{2}^{2}-M_{1}^{2}=\left|M_{1} \Gamma_{1}-M_{2} \Gamma_{2}\right|$, i.e. when the mass difference is of the order of the difference of the decay widths of the neutrinos. The maximal value is given by

$$
R_{\max }^{\text {Boltzmann }}=\frac{M_{1} M_{2}}{2\left|M_{1} \Gamma_{1}-M_{2} \Gamma_{2}\right|} .
$$


Since the decay widths of the neutrinos are suppressed by the Yukawa couplings, this ratio can be much larger than one and can compensate the one-loop suppression of the $C P$-violating rates, so that it is possible to lower the scale of leptogenesis considerably below $10^{9} \mathrm{GeV}$ [10]. In addition, it has been noticed that apparently a further enhancement occurs when not only the masses of the neutrinos are quasi-degenerate, but also their decay widths $\Gamma_{1}$ and $\Gamma_{2}$ are of similar size, so that $R_{\max } \propto\left|M_{1} \Gamma_{1}-M_{2} \Gamma_{2}\right|^{-1}$ is further enhanced. Phenomenologically, this situation could occur when the degeneracy of the masses and of the couplings originates from an underlying flavor symmetry (see e.g. $[66])$.

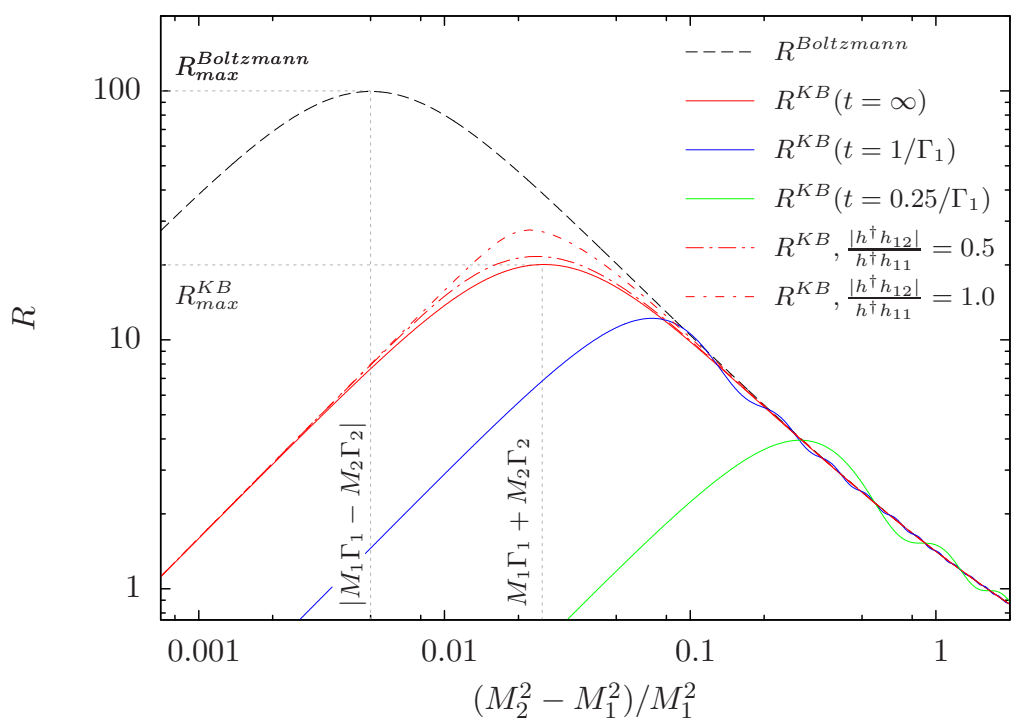

Figure 5: Function $R$ characterizing the amount of resonant enhancement. Shown is the dependence on the mass-squared splitting $M_{2}^{2}-M_{1}^{2}$ for the Boltzmann result (dashed) and for the result obtained based on Kadanoff-Baym equations, for three fixed times $t=0.25 / \Gamma_{1}, 1 / \Gamma_{1}, \infty$ (solid lines). The widths are chosen as in Fig. 4. The dotted lines indicate the maximal resonant enhancement as predicted by the Boltzmann and Kadanoff-Baym results, respectively. The dot-dashed lines shows the numerical result obtained for large off-diagonal Yukawa couplings $\left|h^{\dagger} h\right|_{12} /\left(h^{\dagger} h\right)_{11}=0.5$ and 1 , respectively. Here $\left(h^{\dagger} h\right)_{i i}$ are chosen as for the analytical result, and $\arg \left(h^{\dagger} h\right)_{12}=\pi / 8$. The numerical and analytical results agree for $\left|h^{\dagger} h\right|_{12} \ll\left|\left(h^{\dagger} h\right)_{22}-\left(h^{\dagger} h\right)_{11}\right|$, as expected.

The Kadanoff-Baym result for the lepton asymmetry is proportional to the function $R^{\text {Boltzmann }}$ as well. However, the additional coherent contributions to the lepton asymmetry have the tendency to cancel the Boltzmann-like contribution. Therefore, in order to determine the maximal possible enhancement, it 
is useful to define a modified, time-dependent generalization by

$$
R^{K B}(t) \equiv R^{\text {Boltzmann }} \times \frac{\sum_{I=1,2} \frac{1-e^{-\Gamma_{p I} t}}{\Gamma_{p I}}-4 \operatorname{Re}\left(\frac{1-e^{-i\left(\omega_{p 1}-\omega_{p 2}\right) t} e^{-\left(\Gamma_{p 1}+\Gamma_{p 2}\right) t / 2}}{\Gamma_{p 1}+\Gamma_{p 2}+2 i\left(\omega_{p 1}-\omega_{p 2}\right)}\right)}{\sum_{I=1,2} \frac{1-e^{-\Gamma_{p I} t}}{\Gamma_{p I}}} .
$$

The dependence of $R^{K B}(t)$ and $R^{\text {Boltzmann }}$ on the mass-squared splitting $M_{2}^{2}-M_{1}^{2}$ is shown in Fig. 5 , for a choice of parameters for which $\Gamma_{2} / \Gamma_{1}=1.5$. We observe that the resonant enhancement obtained in the Kadanoff-Baym analysis is smaller compared to the one predicted by the Boltzmann approach, in accordance with the above discussion. In order to identify the maximal possible enhancement, it is instructive to consider the value of $R^{K B}(t)$ for times $t \gtrsim 1 / \Gamma_{p I}$,

$$
\begin{aligned}
\left.R^{K B}(t)\right|_{t \gtrsim 1 / \Gamma_{p I}} & =R^{\text {Boltzmann }} \times \frac{\left(\omega_{p 1}-\omega_{p 2}\right)^{2}+\left(\Gamma_{p 1}-\Gamma_{p 2}\right)^{2} / 4}{\left(\omega_{p 1}-\omega_{p 2}\right)^{2}+\left(\Gamma_{p 1}+\Gamma_{p 2}\right)^{2} / 4} \\
& \simeq R^{\text {Boltzmann }} \times \frac{\left(M_{2}^{2}-M_{1}^{2}\right)^{2}+\left(M_{1} \Gamma_{1}-M_{2} \Gamma_{2}\right)^{2}}{\left(M_{2}^{2}-M_{1}^{2}\right)^{2}+\left(M_{1} \Gamma_{1}+M_{2} \Gamma_{2}\right)^{2}} .
\end{aligned}
$$

In the last line we have used that for quasi-degenerate Majorana masses $\Gamma_{p I}=$ $M_{I} \Gamma_{I} / \omega_{p I} \simeq M_{I} \Gamma_{I} / \omega_{p}$ and $\omega_{p 1}-\omega_{p 2} \simeq\left(M_{1}^{2}-M_{2}^{2}\right) /\left(2 \omega_{p}\right)$. Note that the ratio of the lepton asymmetries at late times $t \gtrsim 1 / \Gamma_{p I}$ is consequently given by

$$
\left.\frac{n_{L}^{K B}(t)}{n_{L}^{B \text { oltzmann }}(t)}\right|_{t \gtrsim 1 / \Gamma_{p I}} \approx \frac{\left(M_{2}^{2}-M_{1}^{2}\right)^{2}+\left(M_{1} \Gamma_{1}-M_{2} \Gamma_{2}\right)^{2}}{\left(M_{2}^{2}-M_{1}^{2}\right)^{2}+\left(M_{1} \Gamma_{1}+M_{2} \Gamma_{2}\right)^{2}} .
$$

Thus, compared to the Boltzmann treatment, the coherent contributions lead to an additional factor that effectively changes the regulator in the resonant term from the difference to the sum of the decay rates. This means that the maximal enhancement of the lepton asymmetry occurs in fact for $M_{2}^{2}-M_{1}^{2}=$ $M_{1} \Gamma_{1}+M_{2} \Gamma_{2}$, and is given by

$$
R_{\max }^{K B}=\frac{M_{1} M_{2}}{2\left(M_{1} \Gamma_{1}+M_{2} \Gamma_{2}\right)} .
$$

Consequently, the coherent contributions have the effect to cut off the resonant enhancement that occurs in the doubly degenerate limit $M_{1} \rightarrow M_{2}$ and $\Gamma_{1} \rightarrow \Gamma_{2}$ within the Boltzmann approach.

As is apparent from the expressions derived above, the maximal resonant enhancement within the Kadanoff-Baym analysis is always smaller compared to the Boltzmann approach. The predictions of both approaches are comparable if either $\Gamma_{2} / \Gamma_{1} \ll 1$ or $\Gamma_{2} / \Gamma_{1} \gg 1$, while the Kadanoff-Baym result is significantly smaller than the Boltzmann result when both decay widths are of comparable size.

\subsection{Comparison of analytical and numerical results}

The explicit analytical solutions obtained in the Kadanoff-Baym approach that have been discussed in this section have been obtained by using a Breit-Wigner approximation and an expansion for small Yukawa couplings that is valid within 
the parameter region $\operatorname{Re}\left(h^{\dagger} h\right)_{12} \lesssim\left|\left(h^{\dagger} h\right)_{22}-\left(h^{\dagger} h\right)_{11}\right|$ and $\left(M_{2}-M_{1}\right) / M_{1} \gtrsim$ $\operatorname{Re}\left(h^{\dagger} h\right)_{12} /(8 \pi)$.

We will now compare the analytical results with a semi-analytical one and then with a numerical approach. The semi-analytical result is also based on the Breit-Wigner approximation. However, the coefficients $F_{I J}$ entering the expression (119) for the lepton asymmetry are computed using the full solutions for the effective masses and widths $\omega_{p I}$ and $\Gamma_{p I}$ given in Eq. (97) as well as the full expressions (93) for the retarded and advanced propagators. Consequently, this semi-analytical approach is valid also when $\operatorname{Re}\left(h^{\dagger} h\right)_{12} \sim\left(h^{\dagger} h\right)_{i i}$ and for mass splittings $\left(M_{2}-M_{1}\right) / M_{1}$ larger or smaller than $\operatorname{Re}\left(h^{\dagger} h\right)_{12} /(8 \pi)$. We have checked that the results obtained in both approaches agree provided that $\left|h^{\dagger} h\right|_{12} \ll\left|\left(h^{\dagger} h\right)_{22}-\left(h^{\dagger} h\right)_{11}\right|$.

The semi-analytical result is shown as dot-dashed lines in Fig. 5 for two choices of $\left|h^{\dagger} h\right|_{12}$. The resonant enhancement becomes slightly stronger when $\left|h^{\dagger} h\right|_{12}$ is increased. However, the mass splitting for which the maximal enhancement occurs remains approximately the same. In addition, a good agreement is found also for extremely small mass splittings. Formally, the validity of the analytical approach requires that $\left(M_{2}-M_{1}\right) / M_{1} \gtrsim \operatorname{Re}\left(h^{\dagger} h\right)_{12} /(8 \pi) \sim 0.005(0.01)$ for $\left|h^{\dagger} h\right|_{12} /\left(h^{\dagger} h\right)_{11}=0.5(1)$. Nevertheless, the comparison with the semianalytical approach indicates that the asymptotic value of the resonant enhancement is predicted correctly also for extremely small mass splittings. It is worth noting that the semi-analytical result can be reproduced rather accurately by replacing the first line of Eq. (125) by

$$
\frac{\operatorname{Im}\left[\left(h^{\dagger} h\right)_{12}^{2}\right]}{8 \pi} \frac{M_{1} M_{2}\left(M_{2}^{2}-M_{1}^{2}\right)}{\left(\left(M_{2}^{\text {pole }}\right)^{2}-\left(M_{1}^{\text {pole }}\right)^{2}\right)^{2}+\left(M_{1}^{\text {pole }} \Gamma_{1}^{\text {pole }}-M_{2}^{\text {pole }} \Gamma_{2}^{\text {pole }}\right)^{2}} .
$$

and using the full expressions for $\omega_{p I}$ and $\Gamma_{p I}$. Here the denominator contains the pole masses and widths discussed in section 4, and the enumerator is proportional to the basis-invariant quantity

$$
\operatorname{Im}\left[\operatorname{tr}\left[h^{\dagger} h M^{\dagger} M M^{\dagger} h^{T} h^{*} M\right]\right]=\operatorname{Im}\left[\left(h^{\dagger} h\right)_{12}^{2}\right] M_{1} M_{2}\left(M_{2}^{2}-M_{1}^{2}\right),
$$

related to $C P$-violation [9]. Since the pole masses and widths agree with the mass and width parameters in the domain of validity of the analytical result, see Eq. (98), this replacement is consistent with the results discussed previously. The corresponding generalization of the enhancement factor leads to

$$
\left.R^{K B}(t)\right|_{t \gtrsim 1 / \Gamma_{p I}} \simeq \frac{M_{1} M_{2}\left(M_{2}^{2}-M_{1}^{2}\right)}{\left(\left(M_{2}^{p o l e}\right)^{2}-\left(M_{1}^{\text {pole }}\right)^{2}\right)^{2}+\left(M_{1}^{\text {pole }} \Gamma_{1}^{p o l e}+M_{2}^{\text {pole }} \Gamma_{2}^{\text {pole }}\right)^{2}} .
$$

This expression reproduces the numerical results shown in Fig. 5 with a very good accuracy. It coincides with the expression obtained from the analytical solution for mass splittings that are large compared to $\operatorname{Re}\left(h^{\dagger} h\right)_{12} /(8 \pi)$, and also in the opposite limit, because then $\Gamma_{1}^{\text {pole }}+\Gamma_{2}^{\text {pole }} \simeq \Gamma_{1}+\Gamma_{2}$, as can be seen from Eq. (99). This behaviour is apparent also in Fig. 5.

In order to cross-check the Breit-Wigner approximation, we have also solved the Kadanoff-Baym equation (31b) for the spectral function of the Majorana neutrinos directly in time representation, for the spatial momentum mode $\mathbf{p}=$ 0 . The contribution from this mode to the lepton asymmetry can then be 
obtained via Eqs. $(76,75)$. We have used the one-loop expression (36) for the self-energy of the Majorana neutrinos, and inserted the thermal lepton and Higgs propagators $(77,78)$. For the numerical analysis it is convenient to use an exponential momentum cutoff $e^{-|\mathbf{q}| / \Lambda}$ for the computation of the leptonHiggs loop integral, and to solve the equation for the bare propagators. The renormalization is then performed by Fourier transforming the regulated loop integral to frequency space, and applying a field renormalization such that the resulting expression matches with the renormalized Schwinger-Dyson Eq. (87). For the cutoff we have used values in the range $\Lambda / M_{1} \sim 3.3-5$. We have checked that our renormalization prescription removes the dependence on the cutoff at a satisfactory level. Furthermore, we have used a time step $a_{t}=0.025 / M_{1}$ and computed the solution for temperatures $T \rightarrow 0$ and $T / M_{1}=0.25$ in the limit $\Gamma_{\ell}, \Gamma_{\phi} \rightarrow 0$. For the renormalized mass and coupling parameters we have chosen the same values that are shown in Fig. 4. We find that the numerical solution for the spectral function can be well described by a sum of exponentially damped oscillating functions with damping rate and frequency in accordance with the Breit-Wigner solution (105). In addition, the resulting lepton asymmetry agrees well with the analytical result for $\left|h^{\dagger} h\right|_{12} \ll\left|\left(h^{\dagger} h\right)_{22}-\left(h^{\dagger} h\right)_{11}\right|$ and with the semi-analytical result for $\operatorname{Re}\left(h^{\dagger} h\right)_{12} \sim\left(h^{\dagger} h\right)_{i i}$.

\section{Conclusions}

In this work we have studied the resonant enhancement of the lepton asymmetry that is generated by the lepton-number violating interaction of lepton and Higgs fields with a pair of quasi-degenerate Majorana neutrino species within a first-principle approach. The quantum-field theoretical treatment of this system allows one to study the resonant enhancement in the case when the mass difference of the heavy neutrino species is comparable to their widths. This regime is of particular interest, because it corresponds to the parameter region for which the resonant enhancement is maximal, and thus determines the amount by which the efficiency of leptogenesis can be increased compared to the case of a hierarchical spectrum of the Majorana neutrinos. By treating the lepton and Higgs fields as a thermal bath and neglecting the backreaction, it was possible to obtain a formal analytical solution for the Kadanoff-Baym equations that describe the non-equilibrium evolution of the coupled system of Majorana neutrinos. We have used this solution in order to analyse the generation of a lepton asymmetry, for which we have obtained approximate analytical as well as numerical results. Finally, we have compared the resonant enhancement that is obtained from the quantum-field theoretical Kadanoff-Baym approach to the classical Boltzmann approach.

We find that for mass splittings of the Majorana neutrinos that are large compared to their widths the lepton asymmetries obtained within the KadanoffBaym and the Boltzmann approach have the same time-dependence, and differ by an overall factor that is related to the finite width of lepton and Higgs fields. When the mass splitting of the Majorana neutrinos is only slightly larger than their widths, the Kadanoff-Baym result is given by a sum of Boltzmann-like contributions and oscillating contributions which can be attributed to coherent transitions between the two Majorana neutrino species. If the mass splitting is of the same order as the width, a cancellation of the Boltzmann-like and coherent 
contributions occurs in the Kadanoff-Baym result and the lepton asymmetry is suppressed compared to the Boltzmann result. We have also compared the analytical approximation for the Kadanoff-Baym result to numerical results, and found good agreement within the domain of validity. In addition, we find that the qualitative behaviour is robust.

Our analytical result confirms the form of the regulator given in Eq. (6). However, the cancellation that arises due to the coherent flavor transitions reduces the maximal possible enhancement. The analytical treatment indicates that the resonant enhancement can be estimated approximately by replacing the 'regulator' according to $M_{1} \Gamma_{1}-M_{2} \Gamma_{2} \rightarrow M_{1} \Gamma_{1}+M_{2} \Gamma_{2}$. The cancellation is particularly important when the widths of the two neutrino species are of comparable size. We therefore expect that the coherent flavor transitions are crucial within phenomenological scenarios for which the masses and the decay rates are quasi-degenerate. However, since the interplay of Boltzmann-like and coherent contributions is a dynamical process, it is necessary to investigate these scenarios in detail. Depending on the considered scenario, it may be appropriate to consider initial conditions for the Majorana neutrinos that are different from the vacuum initial conditions considered here, which will also have an impact on the asymmetry. In addition, we note that it would be interesting to include the back-reaction and to implement the gauge interactions of lepton and Higgs fields in a systematic way. Nevertheless, we expect that the partial cancellation of the final asymmetry due to coherence effects is a generical result.

\section{Note added}

The authors of Ref. [67], which appeared simultaneously to this one, independently considered a description of leptogenesis in the resonant regime using closed time path techniques, but working in Wigner space.

\section{Acknowledgements}

MG is grateful to Wilfried Buchmüller, Marco Drewes, Clemens Kiessig, Sebastian Mendizabal, Markus Michael Müller and Christoph Weniger for valuable discussions and comments. The work of MG was partially supported by the DFG cluster of excellence "Origin and Structure of the Universe." AK is supported by DFG under Grant KA-3274/1-1 "Systematic analysis of baryogenesis in non-equilibrium quantum field theory".

\section{A Expansion in the Yukawa couplings}

In this Appendix approximate analytical expressions for the coefficients $F_{I J}$ defined in Eq. (117) are derived, under the assumptions discussed in section 4.3. These coefficients determine the resonant enhancement of the lepton asymmetry $n_{L}(t)$ that is generated by Majorana neutrinos interacting with a thermal bath, according to Eq. (119).

Within the parameter region described in section 4.3, the complex poles of the retarded and advanced Majorana neutrino two-point functions obtained at 
leading order in the Yukawa couplings are given by Eq. (98),

$$
x_{I}=M_{I}^{2}-i M_{I} \Gamma_{I}, \quad I=1,2 .
$$

For the residues of the poles we find in the same approximation,

$$
\begin{aligned}
Z_{1 R}= & -\left(\begin{array}{cc}
M_{1}+\not p & 0 \\
0 & 0
\end{array}\right)+\frac{1}{x_{1}-x_{2}}\left\{i\left(M_{1}^{2}-M_{2}^{2}\right) \frac{\Gamma_{1}}{2 M_{1}}\left(\begin{array}{cc}
2 M_{1}+\not p & 0 \\
0 & 0
\end{array}\right)\right. \\
& +i\left(M_{1}+M_{2}\right)\left(M_{2} \Gamma_{1}-M_{1} \Gamma_{2}\right) \frac{\tan (2 \theta)}{4 M_{2}}\left(\begin{array}{cc}
0 & M_{1}+\not p \\
M_{1}+\not p & 0
\end{array}\right) \\
& \left.+\left(\operatorname{Im} \gamma_{12}\right) M_{1}\left(M_{1}-M_{2}\right)\left(\begin{array}{cc}
0 & \gamma_{5}\left(M_{1}-\not p\right) \\
\gamma_{5}\left(M_{1}+\not p\right) & 0
\end{array}\right)\right\}, \\
Z_{2 R}= & -\left(\begin{array}{cc}
0 & 0 \\
0 & M_{2}+\not p
\end{array}\right)+\frac{1}{x_{1}-x_{2}}\left\{i\left(M_{1}^{2}-M_{2}^{2}\right) \frac{\Gamma_{2}}{2 M_{2}}\left(\begin{array}{cc}
0 & 0 \\
0 & 2 M_{2}+\not p
\end{array}\right)\right. \\
& -i\left(M_{1}+M_{2}\right)\left(M_{2} \Gamma_{1}-M_{1} \Gamma_{2}\right) \frac{\tan (2 \theta)}{4 M_{1}}\left(\begin{array}{cc}
0 & M_{2}+\not p \\
M_{2}+\not p & 0
\end{array}\right) \\
& \left.-\left(\operatorname{Im} \gamma_{12}\right) M_{2}\left(M_{1}-M_{2}\right)\left(\begin{array}{cc}
0 & \gamma_{5}\left(M_{2}+\not p\right) \\
\gamma_{5}\left(M_{2}-\not p\right) & 0
\end{array}\right)\right\} .
\end{aligned}
$$

The positive and negative frequency coefficients of the Fourier transformed retarded and advanced propagators can then be obtained using Eq.(106). At zeroth order in the Yukawa couplings, the coefficients reduce to

$$
\begin{aligned}
S_{1 R}^{-} & \rightarrow S_{1 A}^{-} \rightarrow \frac{i}{2} \frac{M_{1}+\omega_{p 1} \gamma_{0}-\mathbf{p} \gamma}{\omega_{p 1}}\left(\begin{array}{ll}
1 & 0 \\
0 & 0
\end{array}\right), \\
S_{1 R}^{+} & \rightarrow S_{1 A}^{+} \rightarrow-\frac{i}{2} \frac{M_{1}-\omega_{p 1} \gamma_{0}-\mathbf{p} \gamma}{\omega_{p 1}}\left(\begin{array}{ll}
1 & 0 \\
0 & 0
\end{array}\right),
\end{aligned}
$$

and the result for $I=2$ is analogous. The leading contributions to the offdiagonal elements arise at first order in $h^{\dagger} h$ and are given by

$$
\begin{aligned}
S_{1 R}^{12-} & =\frac{M_{1}}{2 \omega_{p 1}} \frac{\left(M_{1}+\omega_{p 1} \gamma_{0}-\mathbf{p} \gamma\right)\left[\Delta \Gamma-\frac{i}{16 \pi} \operatorname{Im}\left(h^{\dagger} h_{12}\right)\left(M_{1}-M_{2}\right) \gamma_{5}\right]}{M_{1}^{2}-M_{2}^{2}-i M_{1} \Gamma_{1}+i M_{2} \Gamma_{2}}, \\
S_{1 R}^{21-} & =\frac{M_{1}}{2 \omega_{p 1}} \frac{\left[\Delta \Gamma-\frac{i}{16 \pi} \operatorname{Im}\left(h^{\dagger} h_{12}\right)\left(M_{1}-M_{2}\right) \gamma_{5}\right]\left(M_{1}+\omega_{p 1} \gamma_{0}-\mathbf{p} \gamma\right)}{M_{1}^{2}-M_{2}^{2}-i M_{1} \Gamma_{1}+i M_{2} \Gamma_{2}}, \\
S_{1 R}^{12+} & =\frac{M_{1}}{2 \omega_{p 1}} \frac{\left(M_{1}-\omega_{p 1} \gamma_{0}-\mathbf{p} \gamma\right)\left[\Delta \Gamma-\frac{i}{16 \pi} \operatorname{Im}\left(h^{\dagger} h_{12}\right)\left(M_{1}-M_{2}\right) \gamma_{5}\right]}{M_{1}^{2}-M_{2}^{2}+i M_{1} \Gamma_{1}-i M_{2} \Gamma_{2}}, \\
S_{1 R}^{21+} & =\frac{M_{1}}{2 \omega_{p 1}} \frac{\left[\Delta \Gamma-\frac{i}{16 \pi} \operatorname{Im}\left(h^{\dagger} h_{12}\right)\left(M_{1}-M_{2}\right) \gamma_{5}\right]\left(M_{1}-\omega_{p 1} \gamma_{0}-\mathbf{p} \gamma\right)}{M_{1}^{2}-M_{2}^{2}+i M_{1} \Gamma_{1}-i M_{2} \Gamma_{2}},
\end{aligned}
$$




$$
\begin{aligned}
& S_{1 A}^{12-}=-\frac{M_{1}}{2 \omega_{p 1}} \frac{\left(M_{1}+\omega_{p 1} \gamma_{0}-\mathbf{p} \gamma\right)\left[\Delta \Gamma-\frac{i}{16 \pi} \operatorname{Im}\left(h^{\dagger} h_{12}\right)\left(M_{1}-M_{2}\right) \gamma_{5}\right]}{M_{1}^{2}-M_{2}^{2}+i M_{1} \Gamma_{1}-i M_{2} \Gamma_{2}}, \\
& S_{1 A}^{21-}=-\frac{M_{1}}{2 \omega_{p 1}} \frac{\left[\Delta \Gamma-\frac{i}{16 \pi} \operatorname{Im}\left(h^{\dagger} h_{12}\right)\left(M_{1}-M_{2}\right) \gamma_{5}\right]\left(M_{1}+\omega_{p 1} \gamma_{0}-\mathbf{p} \gamma\right)}{M_{1}^{2}-M_{2}^{2}+i M_{1} \Gamma_{1}-i M_{2} \Gamma_{2}}, \\
& S_{1 A}^{12+}=-\frac{M_{1}}{2 \omega_{p 1}} \frac{\left(M_{1}-\omega_{p 1} \gamma_{0}-\mathbf{p} \gamma\right)\left[\Delta \Gamma-\frac{i}{16 \pi} \operatorname{Im}\left(h^{\dagger} h_{12}\right)\left(M_{1}-M_{2}\right) \gamma_{5}\right]}{M_{1}^{2}-M_{2}^{2}-i M_{1} \Gamma_{1}+i M_{2} \Gamma_{2}}, \\
& S_{1 A}^{21+}=-\frac{M_{1}}{2 \omega_{p 1}} \frac{\left[\Delta \Gamma-\frac{i}{16 \pi} \operatorname{Im}\left(h^{\dagger} h_{12}\right)\left(M_{1}-M_{2}\right) \gamma_{5}\right]\left(M_{1}-\omega_{p 1} \gamma_{0}-\mathbf{p} \gamma\right)}{M_{1}^{2}-M_{2}^{2}-i M_{1} \Gamma_{1}+i M_{2} \Gamma_{2}}, \\
& S_{2 R}^{12-}=-\frac{M_{2}}{2 \omega_{p 2}} \frac{\left[\Delta \Gamma-\frac{i}{16 \pi} \operatorname{Im}\left(h^{\dagger} h_{12}\right)\left(M_{1}-M_{2}\right) \gamma_{5}\right]\left(M_{2}+\omega_{p 2} \gamma_{0}-\mathbf{p} \gamma\right)}{M_{1}^{2}-M_{2}^{2}-i M_{1} \Gamma_{1}+i M_{2} \Gamma_{2}}, \\
& S_{2 R}^{21-}=-\frac{M_{2}}{2 \omega_{p 2}} \frac{\left(M_{2}+\omega_{p 2} \gamma_{0}-\mathbf{p} \gamma\right)\left[\Delta \Gamma-\frac{i}{16 \pi} \operatorname{Im}\left(h^{\dagger} h_{12}\right)\left(M_{1}-M_{2}\right) \gamma_{5}\right]}{M_{1}^{2}-M_{2}^{2}-i M_{1} \Gamma_{1}+i M_{2} \Gamma_{2}}, \\
& S_{2 R}^{12+}=-\frac{M_{2}}{2 \omega_{p 2}} \frac{\left[\Delta \Gamma-\frac{i}{16 \pi} \operatorname{Im}\left(h^{\dagger} h_{12}\right)\left(M_{1}-M_{2}\right) \gamma_{5}\right]\left(M_{2}-\omega_{p 2} \gamma_{0}-\mathbf{p} \gamma\right)}{M_{1}^{2}-M_{2}^{2}+i M_{1} \Gamma_{1}-i M_{2} \Gamma_{2}}, \\
& S_{2 R}^{21+}=-\frac{M_{2}}{2 \omega_{p 2}} \frac{\left(M_{2}-\omega_{p 2} \gamma_{0}-\mathbf{p} \gamma\right)\left[\Delta \Gamma-\frac{i}{16 \pi} \operatorname{Im}\left(h^{\dagger} h_{12}\right)\left(M_{1}-M_{2}\right) \gamma_{5}\right]}{M_{1}^{2}-M_{2}^{2}+i M_{1} \Gamma_{1}-i M_{2} \Gamma_{2}}, \\
& S_{2 A}^{12-}=\frac{M_{2}}{2 \omega_{p 2}} \frac{\left[\Delta \Gamma-\frac{i}{16 \pi} \operatorname{Im}\left(h^{\dagger} h_{12}\right)\left(M_{1}-M_{2}\right) \gamma_{5}\right]\left(M_{2}+\omega_{p 2} \gamma_{0}-\mathbf{p} \gamma\right)}{M_{1}^{2}-M_{2}^{2}+i M_{1} \Gamma_{1}-i M_{2} \Gamma_{2}}, \\
& S_{2 A}^{21-}=\frac{M_{2}}{2 \omega_{p 2}} \frac{\left(M_{2}+\omega_{p 2} \gamma_{0}-\mathbf{p} \gamma\right)\left[\Delta \Gamma-\frac{i}{16 \pi} \operatorname{Im}\left(h^{\dagger} h_{12}\right)\left(M_{1}-M_{2}\right) \gamma_{5}\right]}{M_{1}^{2}-M_{2}^{2}+i M_{1} \Gamma_{1}-i M_{2} \Gamma_{2}}, \\
& S_{2 A}^{12+}=\frac{M_{2}}{2 \omega_{p 2}} \frac{\left[\Delta \Gamma-\frac{i}{16 \pi} \operatorname{Im}\left(h^{\dagger} h_{12}\right)\left(M_{1}-M_{2}\right) \gamma_{5}\right]\left(M_{2}-\omega_{p 2} \gamma_{0}-\mathbf{p} \gamma\right)}{M_{1}^{2}-M_{2}^{2}-i M_{1} \Gamma_{1}+i M_{2} \Gamma_{2}}, \\
& S_{2 A}^{21+}=\frac{M_{2}}{2 \omega_{p 2}} \frac{\left(M_{2}-\omega_{p 2} \gamma_{0}-\mathbf{p} \gamma\right)\left[\Delta \Gamma-\frac{i}{16 \pi} \operatorname{Im}\left(h^{\dagger} h_{12}\right)\left(M_{1}-M_{2}\right) \gamma_{5}\right]}{M_{1}^{2}-M_{2}^{2}-i M_{1} \Gamma_{1}+i M_{2} \Gamma_{2}},
\end{aligned}
$$

where

$$
\Delta \Gamma \equiv\left(M_{1}+M_{2}\right) \frac{\operatorname{Re}\left(h^{\dagger} h_{12}\right)}{16 \pi} .
$$

The $C P$ conjugated propagators differ by the sign of $\operatorname{Im}\left(h^{\dagger} h_{12}\right)$.

Using the vacuum initial condition (124) for the statistical propagator in leading order in the Yukawa couplings we find for the coefficients $F_{J I}$ :

$$
\begin{aligned}
F_{J I}^{\epsilon}= & f_{F D}\left(\omega_{p 1}\right) \operatorname{tr}\left[\frac{M_{1}-\mathbf{p} \gamma}{\omega_{p 1}}\left(\gamma_{J I}^{11 \epsilon}-\bar{\gamma}_{J I}^{11 \epsilon}\right)\right] \\
& +f_{F D}\left(\omega_{p 2}\right) \operatorname{tr}\left[\frac{M_{2}-\mathbf{p} \gamma}{\omega_{p 2}}\left(\gamma_{J I}^{22 \epsilon}-\bar{\gamma}_{J I}^{22 \epsilon}\right)\right],
\end{aligned}
$$

Lets consider for concreteness the first contribution. At leading order in Yukawas,

$$
\begin{aligned}
& \gamma_{J I}^{11 \epsilon}-\bar{\gamma}_{J I}^{11 \epsilon}=-i \gamma_{0} S_{A J}^{11 \epsilon} P_{L} S_{\ell \phi}^{\epsilon \epsilon} P_{R} \Delta S_{R I}^{\epsilon} \gamma_{0} \\
& -i \gamma_{0} \Delta S_{A J}^{\epsilon} P_{L} S_{\ell \phi}^{\epsilon \epsilon} P_{\mathbf{p}} P_{R} S_{R I}^{11 \epsilon} \gamma_{0} \text {, }
\end{aligned}
$$


where

$$
\begin{aligned}
& \Delta S_{R I}^{\epsilon} \equiv \operatorname{Re}\left(h^{\dagger} h\right)_{12}\left(S_{R I}^{21 \epsilon}-\bar{S}_{R I}^{21 \epsilon}\right)+i \operatorname{Im}\left(h^{\dagger} h\right)_{12}\left(S_{R I}^{21 \epsilon}+\bar{S}_{R I}^{21 \epsilon}\right), \\
& \Delta S_{A I}^{\epsilon} \equiv \operatorname{Re}\left(h^{\dagger} h\right)_{12}\left(S_{A I}^{12 \epsilon}-\bar{S}_{A I}^{12 \epsilon}\right)-i \operatorname{Im}\left(h^{\dagger} h\right)_{12}\left(S_{A I}^{12 \epsilon}+\bar{S}_{A I}^{12 \epsilon}\right) .
\end{aligned}
$$

From the explicit expressions for $S_{R(A) I}^{i j \epsilon}$ given above, one obtains

$$
\begin{aligned}
\Delta S_{R 1}^{+}= & i \frac{\operatorname{Im}\left[\left(h^{\dagger} h\right)_{12}^{2}\right]}{16 \pi} \frac{M_{1}}{\omega_{p 1}} \frac{\left\{M_{1} P_{L}+M_{2} P_{R}\right\}\left[M_{1}-\omega_{p 1} \gamma_{0}-\mathbf{p} \gamma\right]}{M_{1}^{2}-M_{2}^{2}+i M_{1} \Gamma_{1}-i M_{2} \Gamma_{2}} \\
\Delta S_{R 1}^{-}= & i \frac{\operatorname{Im}\left[\left(h^{\dagger} h\right)_{12}^{2}\right]}{16 \pi} \frac{M_{1}}{\omega_{p 1}} \frac{\left\{M_{1} P_{L}+M_{2} P_{R}\right\}\left[M_{1}+\omega_{p 1} \gamma_{0}-\mathbf{p} \gamma\right]}{M_{1}^{2}-M_{2}^{2}-i M_{1} \Gamma_{1}+i M_{2} \Gamma_{2}} \\
\Delta S_{R 2}^{+}= & -i \frac{\operatorname{Im}\left[\left(h^{\dagger} h\right)_{12}^{2}\right]}{16 \pi} \frac{M_{2}}{\omega_{p 2}} \frac{\left[M_{2}-\omega_{p 2} \gamma_{0}-\mathbf{p} \gamma\right]\left\{M_{1} P_{L}+M_{2} P_{R}\right\}}{M_{1}^{2}-M_{2}^{2}+i M_{1} \Gamma_{1}-i M_{2} \Gamma_{2}} \\
\Delta S_{R 2}^{-}= & -i \frac{\operatorname{Im}\left[\left(h^{\dagger} h\right)_{12}^{2}\right]}{16 \pi} \frac{M_{2}}{\omega_{p 2}} \frac{\left[M_{2}+\omega_{p 2} \gamma_{0}-\mathbf{p} \gamma\right]\left\{M_{1} P_{L}+M_{2} P_{R}\right\}}{M_{1}^{2}-M_{2}^{2}-i M_{1} \Gamma_{1}+i M_{2} \Gamma_{2}} \\
\Delta S_{A 1}^{+}= & i \frac{\operatorname{Im}\left[\left(h^{\dagger} h\right)_{12}^{2}\right]}{16 \pi} \frac{M_{1}}{\omega_{p 1}} \frac{\left[M_{1}-\omega_{p 1} \gamma_{0}-\mathbf{p} \gamma\right]\left\{M_{1} P_{R}+M_{2} P_{L}\right\}}{M_{1}^{2}-M_{2}^{2}-i M_{1} \Gamma_{1}+i M_{2} \Gamma_{2}} \\
\Delta S_{A 1}^{-}= & i \frac{\operatorname{Im}\left[\left(h^{\dagger} h\right)_{12}^{2}\right]}{16 \pi} \frac{M_{1}}{\omega_{p 1}} \frac{\left[M_{1}+\omega_{p 1} \gamma_{0}-\mathbf{p} \gamma\right]\left\{M_{1} P_{R}+M_{2} P_{L}\right\}}{M_{1}^{2}-M_{2}^{2}+i M_{1} \Gamma_{1}-i M_{2} \Gamma_{2}} \\
\Delta S_{A 2}^{+}= & -i \frac{\operatorname{Im}\left[\left(h^{\dagger} h\right)_{12}^{2}\right]}{16 \pi} \frac{M_{2}}{\omega_{p 2}} \frac{\left\{M_{1} P_{R}+M_{2} P_{L}\right\}\left[M_{2}-\omega_{p 2} \gamma_{0}-\mathbf{p} \gamma\right]}{M_{1}^{2}-M_{2}^{2}-i M_{1} \Gamma_{1}+i M_{2} \Gamma_{2}} \\
\Delta S_{A 2}^{-}= & -i \frac{\operatorname{Im}\left[\left(h^{\dagger} h\right)_{12}^{2}\right]}{16 \pi} \frac{M_{2}}{\omega_{p 2}} \frac{\left\{M_{1} P_{R}+M_{2} P_{L}\right\}\left[M_{2}+\omega_{p 2} \gamma_{0}-\mathbf{p} \gamma\right]}{M_{1}^{2}-M_{2}^{2}+i M_{1} \Gamma_{1}-i M_{2} \Gamma_{2}} \\
& S_{A J}^{11+}=S_{R J}^{11+}=-\frac{i}{2} \delta_{1 J} \frac{M_{1}-\omega_{p 1} \gamma_{0}-\mathbf{p} \gamma}{\omega_{p 1}} \\
& S_{A J}^{11-}=S_{R J}^{11-}=\frac{i}{2} \delta_{1 J} \frac{M_{1}+\omega_{p 1} \gamma_{0}-\mathbf{p} \gamma}{\omega_{p 1}}
\end{aligned}
$$

Finally, we obtain the following result for the coefficients $F_{J I}^{\epsilon}$ :

$$
\begin{gathered}
F_{11}^{+}=F_{11}^{-}=-\frac{\left(k \omega_{p 1}-\mathbf{k} p\right) f_{\ell \phi}(k, q) f_{F D}\left(\omega_{p 1}\right)}{2 k \omega_{p 1}} \frac{\operatorname{Im}\left[\left(h^{\dagger} h\right)_{12}^{2}\right]}{4 \pi} \\
\times \operatorname{Re} \frac{M_{1} M_{2}}{M_{1}^{2}-M_{2}^{2}+i M_{1} \Gamma_{1}-i M_{2} \Gamma_{2}}, \\
F_{22}^{+}=F_{22}^{-}=-\frac{\left(k \omega_{p 2}-\mathbf{k} p\right) f_{\ell \phi}(k, q) f_{F D}\left(\omega_{p 2}\right)}{2 k \omega_{p 2}} \frac{\operatorname{Im}\left[\left(h^{\dagger} h\right)_{12}^{2}\right]}{4 \pi} \\
\times \operatorname{Re} \frac{M_{1} M_{2}}{M_{1}^{2}-M_{2}^{2}+i M_{1} \Gamma_{1}-i M_{2} \Gamma_{2}}, \\
F_{12}^{ \pm}=\frac{\left(\omega_{p 1}+\omega_{p 2}\right) f_{\ell \phi}(k, q)}{4 k \omega_{p 1} \omega_{p 2}} \frac{\operatorname{Im}\left[\left(h^{\dagger} h\right)_{12}^{2}\right]}{4 \pi} \\
\times \frac{1}{2}\left\{\frac{M_{1} M_{2}\left(k \omega_{p 2}-\mathbf{k} p\right) f_{F D}\left(\omega_{p 1}\right)}{M_{1}^{2}-M_{2}^{2} \pm i M_{1} \Gamma_{1} \mp i M_{2} \Gamma_{2}}+\frac{M_{1} M_{2}\left(k \omega_{p 1}-\mathbf{k} p\right) f_{F D}\left(\omega_{p 2}\right)}{M_{1}^{2}-M_{2}^{2} \mp i M_{1} \Gamma_{1} \pm i M_{2} \Gamma_{2}}\right\},
\end{gathered}
$$




$$
\begin{aligned}
F_{21}^{ \pm}= & \frac{\left(\omega_{p 1}+\omega_{p 2}\right) f_{\ell \phi}(k, q)}{4 k \omega_{p 1} \omega_{p 2}} \frac{\operatorname{Im}\left[\left(h^{\dagger} h\right)_{12}^{2}\right]}{4 \pi} \\
& \times \frac{1}{2}\left\{\frac{M_{1} M_{2}\left(k \omega_{p 2}-\mathbf{k} p\right) f_{F D}\left(\omega_{p 1}\right)}{M_{1}^{2}-M_{2}^{2} \mp i M_{1} \Gamma_{1} \pm i M_{2} \Gamma_{2}}+\frac{M_{1} M_{2}\left(k \omega_{p 1}-\mathbf{k} p\right) f_{F D}\left(\omega_{p 2}\right)}{M_{1}^{2}-M_{2}^{2} \pm i M_{1} \Gamma_{1} \mp i M_{2} \Gamma_{2}}\right\} .
\end{aligned}
$$

\section{B Washout}

In this section we show how to derive washout terms that describe the washout of the lepton asymmetry due to decays and inverse decays from the equation of motion for the lepton asymmetry discussed in Section 3. Formally, these terms arise when expanding Eq. (48) in next-to-leading in the CP-odd parts $\delta S_{\ell}$ and $\delta \Delta_{\phi}$ of lepton and Higgs propagators. Inserting the expansion Eq. (52) as well as the one-loop expression Eq. (34) for the lepton self-energy into Eq. (48), one finds

$$
\begin{aligned}
\left.\frac{d n_{L_{\alpha \beta}(t)}}{d t}\right|_{\text {Washout }}= & \frac{h_{\alpha i} h_{j \beta}^{\dagger}}{2 V} \int d^{3} x \int_{\mathcal{C}} d^{4} y \operatorname{tr}\left[P_{R}\left(S^{i j}(x, y)+\bar{S}^{j i}(x, y)\right) P_{L}\right. \\
& \left.\times\left(\delta S_{\ell}(y, x) \Delta_{\phi}(y, x)+S_{\ell}(y, x) \delta \Delta_{\phi}(y, x)\right)\right] . \quad(141)
\end{aligned}
$$

The CP-odd part of lepton and Higgs propagators can be obtained by solving the corresponding Kadanoff-Baym equations (30a). A common assumption is that Standard Model gauge interactions are able to maintain chemical and kinetic equilibrium, such that the two-point functions can be characterized by a temperature and a chemical potential. The leading contribution to the CP-odd part is obtained by expanding in the chemical potential, which yields in the time/momentum representation

$$
\begin{aligned}
\delta \Delta_{\phi_{\mathbf{q} F}}\left(t, t^{\prime}\right) & =-\frac{2 i \mu_{\phi}}{T} \Delta_{\phi_{\mathbf{q} \rho}}\left(t-t^{\prime}\right) f_{\phi}(q)\left(1+f_{\phi}(q)\right), \\
\delta S_{\ell \mathbf{k} F}\left(t, t^{\prime}\right) & =\frac{2 i \mu_{\ell}}{T} S_{\ell \mathbf{k} \rho}\left(t-t^{\prime}\right) f_{\ell}(k)\left(1-f_{\ell}(k)\right),
\end{aligned}
$$

while the CP-odd part of the spectral $(\rho)$ components vanishes at this order. By inserting the propagators $(77,78)$, the CP-odd part of the lepton-Higgs loop can be written as

$$
\begin{aligned}
\delta S_{\ell \phi_{\mathbf{p}}}\left(t, t^{\prime}\right) & =\int \frac{d^{3} q}{(2 \pi)^{3}}\left(\delta S_{\ell \mathbf{k}}\left(t, t^{\prime}\right) \Delta_{\phi_{\mathbf{q}}}\left(t, t^{\prime}\right)+S_{\ell \mathbf{k}}\left(t, t^{\prime}\right) \delta \Delta_{\phi_{\mathbf{q}}}\left(t, t^{\prime}\right)\right) \\
& \equiv \frac{2 i \mu_{\ell}}{T} \sum_{\epsilon_{i}= \pm} \int \frac{d^{3} q}{(2 \pi)^{3} 2 q} \delta S_{\ell \phi}^{\epsilon_{1} \epsilon_{2}} e^{i\left(\epsilon_{1} k+\epsilon_{2} q\right)\left(t-t^{\prime}\right)-\Gamma_{\ell \phi}\left|t-t^{\prime}\right|}
\end{aligned}
$$

The coefficients for the statistical and spectral components are given by

$$
\begin{aligned}
\delta S_{\ell \phi F}^{\epsilon \epsilon}= & \frac{i}{4}\left(\gamma_{0}+\epsilon \frac{\mathbf{k} \gamma}{k}\right)\left[\left(1+2 f_{\phi}(q)\right) f_{\ell}(k)\left(1-f_{\ell}(k)\right)\right. \\
& \left.-c_{\ell \phi}\left(1-2 f_{\ell}(k)\right) f_{\phi}(q)\left(1+f_{\phi}(q)\right)\right], \\
\delta S_{\ell \phi_{\rho}}^{\epsilon \epsilon}= & \frac{\epsilon}{2}\left(\gamma_{0}+\epsilon \frac{\mathbf{k} \gamma}{k}\right)\left[f_{\ell}(k)\left(1-f_{\ell}(k)\right)-c_{\ell \phi} f_{\phi}(q)\left(1+f_{\phi}(q)\right)\right],
\end{aligned}
$$


where we have defined $c_{\ell \phi} \equiv \mu_{\phi} / \mu_{\ell}$. Inserting in addition the thermal part of the Majorana propagator in Breit-Wigner approximation (105), one finds for the washout term of the total lepton asymmetry

$$
\begin{aligned}
\left.\frac{d n_{L}(t)}{d t}\right|_{\text {Washout }} \simeq & -\frac{\mu_{\ell}}{T} \int \frac{d^{3} p}{(2 \pi)^{3}} \int \frac{d^{3} q}{(2 \pi)^{3} 2 q} \frac{i \epsilon\left(h^{\dagger} h\right)_{j i}}{\omega_{p I}-\epsilon_{1} \epsilon k-\epsilon_{2} \epsilon q+i \epsilon \frac{\Gamma_{\ell \phi}+\Gamma_{p I}}{2}} \\
& \operatorname{tr}\left[P_{R}\left(S_{I R}^{\epsilon i j}+\bar{S}_{I R}^{\epsilon j i}\right) P_{L} \delta S_{\ell \phi_{F}}^{\epsilon_{1} \epsilon_{2}}-P_{R}\left(S_{I F}^{\epsilon i j}+\bar{S}_{I F}^{\epsilon j i}\right) P_{L} \delta S_{\ell \phi_{\rho}}^{\epsilon_{1} \epsilon_{2}}\right] .
\end{aligned}
$$

By relating the chemical potential to the lepton asymmetry, $\mu_{\ell}=3 n_{L} / T^{2}[68]$, one obtains the usual linear dependence for the washout term. The leading contribution arises for $\epsilon=\epsilon_{1}=\epsilon_{2}$, for which the real part of the denominator can become zero. A more explicit analytical form can be obtained by inserting the mode coefficients obtained from expanding in leading order in the Yukawa couplings, as discussed in Section 4.3. The result is

$$
\begin{aligned}
\left.\frac{d n_{L}(t)}{d t}\right|_{\text {Washout }} \simeq & -\frac{\mu_{\ell}\left(1+c_{\ell \phi}\right)}{T} \int \frac{d^{3} p}{(2 \pi)^{3} 2 \omega_{p i}} \int \frac{d^{3} q}{(2 \pi)^{3} 2 q} \int \frac{d^{3} k}{(2 \pi)^{3} 2 k} \\
& \times(2 \pi)^{3} \delta(\mathbf{p}-\mathbf{k}-\mathbf{q}) \times \frac{\Gamma_{\ell \phi}}{\left(\omega_{p i}-k-q\right)^{2}+\Gamma_{\ell \phi}^{2} / 4} \\
& \times 4 k \cdot p_{i}\left(h^{\dagger} h\right)_{i i} f_{N_{i}}^{e q}\left(1-f_{N_{i}}^{e q}\right)\left(1-f_{\ell}(k)+f_{\phi}(q)\right) .
\end{aligned}
$$

In the narrow-width limit $\Gamma_{\ell \phi} \rightarrow 0$, and when neglecting quantum statistical corrections as well as the Higgs asymmetry $\left(c_{\ell \phi} \simeq 0\right)$, the washout term reduces to the well-known form, see e.g. [25, 69, 68].

\section{References}

[1] E. W. Kolb and M. S. Turner, The Early universe (Addison-Wesley (Frontiers in physics, 69), Redwood City, CA, 1990).

[2] G. Hinshaw et al., Five-Year Wilkinson Microwave Anisotropy Probe (WMAP) Observations:Data Processing, Sky Maps, \&3 Basic Results, Astrophys. J. Suppl. 180, 225-245 (2009), 0803.0732.

[3] E. Komatsu et al., Five-Year Wilkinson Microwave Anisotropy Probe (WMAP) Observations:Cosmological Interpretation, Astrophys. J. Suppl. 180, 330-376 (2009), 0803.0547.

[4] A. D. Sakharov, VIOLATION OF CP INVARIANCE, C ASYMMETRY, AND BARYON ASYMMETRY OF THE UNIVERSE, JETP Letters 5, 24-27 (1967).

[5] M. Fukugita and T. Yanagida, Baryogenesis Without Grand Unification, Phys. Lett. B174, 45 (1986).

[6] V. A. Kuzmin, V. A. Rubakov and M. E. Shaposhnikov, On the anomalous electroweak baryon number nonconservation in the early universe, Phys. Lett. B 155, 36 (1985). 
[7] M. Flanz, E. A. Paschos and U. Sarkar, Baryogenesis from a lepton asymmetric universe, Phys.Lett. B345, 248-252 (1995; Phys. Lett. B384 (1996) 487 (erratum)), hep-ph/9411366.

[8] L. Covi, E. Roulet and F. Vissani, CP violating decays in leptogenesis scenarios, Phys.Lett. B384, 169-174 (1996), hep-ph/9605319.

[9] A. Pilaftsis, CP violation and baryogenesis due to heavy Majorana neutrinos, Phys. Rev. D56, 5431-5451 (1997), hep-ph/9707235.

[10] A. Pilaftsis and T. E. J. Underwood, Resonant leptogenesis, Nucl. Phys. B692, 303-345 (2004), hep-ph/0309342.

[11] A. Pilaftsis and T. E. J. Underwood, Electroweak-scale resonant leptogenesis, Phys. Rev. D 72, 113001 (2005), hep-ph/0506107.

[12] S. Antusch, S. Blanchet, M. Blennow and E. Fernandez-Martinez, Nonunitary Leptonic Mixing and Leptogenesis, JHEP 1001, 017 (2010), 0910.5957.

[13] S. Antusch, M. Blennow, E. Fernandez-Martinez and J. Lopez-Pavon, Probing non-unitary mixing and CP-violation at a Neutrino Factory, Phys.Rev. D80, 033002 (2009), 0903.3986.

[14] F. Vissani, Do experiments suggest a hierarchy problem?, Phys.Rev. D57, 7027-7030 (1998), hep-ph/9709409.

[15] M. Flanz, E. A. Paschos, U. Sarkar and J. Weiss, Baryogenesis through mixing of heavy Majorana neutrinos, Phys.Lett. B389, 693-699 (1996), hep-ph/9607310.

[16] J. Liu and G. Segre, Unstable particle mixing and CP violation in weak decays, Phys.Rev. D49, 1342-1349 (1994), hep-ph/9310248.

[17] L. Covi and E. Roulet, Baryogenesis from mixed particle decays, Phys.Lett. B399, 113-118 (1997), hep-ph/9611425.

[18] S. Blanchet, Z. Chacko, S. S. Granor and R. N. Mohapatra, Probing Resonant Leptogenesis at the LHC, Phys.Rev. D82, 076008 (2010), 0904.2174.

[19] A. Pilaftsis, Resonant CP violation induced by particle mixing in transition amplitudes, Nucl. Phys. B504, 61-107 (1997), hep-ph/9702393.

[20] A. Pilaftsis, Heavy Majorana neutrinos and baryogenesis, Int.J.Mod.Phys. A14, 1811-1858 (1999), hep-ph/9812256.

[21] W. Buchmuller and M. Plumacher, CP asymmetry in Majorana neutrino decays, Phys.Lett. B431, 354-362 (1998), hep-ph/9710460.

[22] A. Anisimov, A. Broncano and M. Plumacher, The CP-asymmetry in resonant leptogenesis, Nucl. Phys. B737, 176-189 (2006), hep-ph/0511248.

[23] R. Rangarajan and H. Mishra, Leptogenesis with heavy Majorana neutrinos revisited, Phys.Rev. D61, 043509 (2000), hep-ph/9908417. 
[24] L. Covi, N. Rius, E. Roulet and F. Vissani, Finite temperature effects on CP violating asymmetries, Phys. Rev. D 57, 93-99 (1998), hep-ph/9704366.

[25] G. F. Giudice, A. Notari, M. Raidal, A. Riotto et al., Towards a complete theory of thermal leptogenesis in the SM and MSSM, Nucl. Phys. B685, 89-149 (2004), hep-ph/0310123.

[26] M. Garny, A. Hohenegger and A. Kartavtsev, Medium corrections to the CP-violating parameter in leptogenesis, Phys. Rev. D81, 085028 (2010), 1002.0331 .

[27] C. P. Kiessig, M. Plumacher and M. H. Thoma, Decay of a Yukawa fermion at finite temperature and applications to leptogenesis, Phys. Rev. D82, 036007 (2010), 1003.3016.

[28] C. Kiessig and M. Plumacher, Hard-Thermal-Loop Corrections in Leptogenesis I: CP-Asymmetries (2011), 1111.1231.

[29] C. Kiessig and M. Plumacher, Hard-Thermal-Loop Corrections in Leptogenesis II: Solving the Boltzmann Equations (2011), 1111.1235.

[30] D. Besak and D. Bodeker, Hard thermal loops for soft or collinear external momenta, JHEP 05, 007 (2010), 1002.0022.

[31] A. Anisimov, D. Besak and D. Bodeker, Thermal production of relativistic Majorana neutrinos: Strong enhancement by multiple soft scattering, JCAP 1103, 042 (2011), 1012.3784.

[32] A. Salvio, P. Lodone and A. Strumia, Towards leptogenesis at NLO: the right-handed neutrino interaction rate (2011), 1106.2814.

[33] M. Laine and Y. Schroder, Thermal right-handed neutrino production rate in the non-relativistic regime (2011), 1112.1205.

[34] W. Buchmuller and S. Fredenhagen, Quantum mechanics of baryogenesis, Phys. Lett. B483, 217-224 (2000), hep-ph/0004145.

[35] A. Anisimov, W. Buchmuller, M. Drewes and S. Mendizabal, Nonequilibrium Dynamics of Scalar Fields in a Thermal Bath, Annals Phys. 324, 1234-1260 (2009), 0812.1934.

[36] A. Anisimov, W. Buchmuller, M. Drewes and S. Mendizabal, Leptogenesis from Quantum Interference in a Thermal Bath, Phys. Rev. Lett. 104, 121102 (2010), 1001.3856.

[37] A. Anisimov, W. Buchmuller, M. Drewes and S. Mendizabal, Quantum Leptogenesis I, Annals Phys. 326, 1998-2038 (2011), 1012.5821.

[38] A. De Simone and A. Riotto, Quantum Boltzmann Equations and Leptogenesis, JCAP 0708, 002 (2007), hep-ph/0703175.

[39] A. De Simone and A. Riotto, On Resonant Leptogenesis, JCAP 0708, 013 (2007), 0705.2183. 
[40] M. Garny, A. Hohenegger, A. Kartavtsev and M. Lindner, Systematic approach to leptogenesis in nonequilibrium QFT: vertex contribution to the CP-violating parameter, Phys. Rev. D80, 125027 (2009), 0909.1559.

[41] M. Garny, A. Hohenegger, A. Kartavtsev and M. Lindner, Systematic approach to leptogenesis in nonequilibrium QFT: self-energy contribution to the CP-violating parameter, Phys. Rev. D81, 085027 (2010), 0911.4122.

[42] M. Garny, A. Hohenegger and A. Kartavtsev, Quantum corrections to leptogenesis from the gradient expansion (2010), 1005.5385.

[43] M. Beneke, B. Garbrecht, M. Herranen and P. Schwaller, Finite Number Density Corrections to Leptogenesis, Nucl. Phys. B838, 1-27 (2010), 1002.1326 .

[44] M. Beneke, B. Garbrecht, C. Fidler, M. Herranen et al., Flavoured Leptogenesis in the CTP Formalism, Nucl. Phys. B843, 177-212 (2011), 1007.4783.

[45] B. Garbrecht, Leptogenesis: The Other Cuts, Nucl. Phys. B847, 350-366 (2011), 1011.3122.

[46] E. K. Akhmedov, V. A. Rubakov and A. Y. Smirnov, Baryogenesis via neutrino oscillations, Phys. Rev. Lett. 81, 1359-1362 (1998), hep-ph/9803255.

[47] T. Asaka and M. Shaposhnikov, The nuMSM, dark matter and baryon asymmetry of the universe, Phys. Lett. B620, 17-26 (2005), hep$\mathrm{ph} / 0505013$.

[48] L. Canetti and M. Shaposhnikov, Baryon Asymmetry of the Universe in the NuMSM, JCAP 1009, 001 (2010), 1006.0133.

[49] A. Boyarsky, O. Ruchayskiy and M. E. Shaposhnikov, The role of sterile neutrinos in cosmology and astrophysics, Ann. Rev. Nucl. Part. Sci. 59, 191-214 (2009), 0901.0011.

[50] G. Sigl and G. Raffelt, General kinetic description of relativistic mixed neutrinos, Nucl.Phys. B406, 423-451 (1993).

[51] J.-S. Gagnon and M. Shaposhnikov, Baryon Asymmetry of the Universe without Boltzmann or Kadanoff-Baym, Phys. Rev. D83, 065021 (2011), 1012.1126.

[52] P. Danielewicz, Quantum Theory of Nonequilibrium Processes. 1, Annals Phys. 152, 239-304 (1984).

[53] J. M. Cornwall, R. Jackiw and E. Tomboulis, Effective Action for Composite Operators, Phys.Rev. D10, 2428-2445 (1974).

[54] B. A. Kniehl and A. Pilaftsis, Mixing Renormalization in Majorana Neutrino Theories, Nucl. Phys. B474, 286-308 (1996), hep-ph/9601390.

[55] J. Berges and J. Cox, Thermalization of quantum fields from time-reversal invariant evolution equations, Phys. Lett. B517, 369-374 (2001), hep$\mathrm{ph} / 0006160$. 
[56] J. Berges, Controlled nonperturbative dynamics of quantum fields out of equilibrium, Nucl. Phys. A699, 847-886 (2002), hep-ph/0105311.

[57] G. Aarts and J. Berges, Classical aspects of quantum fields far from equilibrium, Phys. Rev. Lett. 88, 041603 (2002), hep-ph/0107129.

[58] J. Berges, S. Borsanyi and J. Serreau, Thermalization of fermionic quantum fields, Nucl. Phys. B660, 51-80 (2003), hep-ph/0212404.

[59] S. Juchem, W. Cassing and C. Greiner, Quantum dynamics and thermalization for out-of-equilibrium phi**4-theory, Phys. Rev. D69, 025006 (2004), hep-ph/0307353.

[60] A. Arrizabalaga, J. Smit and A. Tranberg, Equilibration in $\varphi^{4}$ theory in 3+1 dimensions, Phys. Rev. D72, 025014 (2005), hep-ph/0503287.

[61] M. Lindner and M. M. Müller, Comparison of Boltzmann equations with quantum dynamics for scalar fields, Phys. Rev. D73, 125002 (2006), hep$\mathrm{ph} / 0512147$.

[62] M. Garny and M. M. Muller, Kadanoff-Baym Equations with Non-Gaussian Initial Conditions: The Equilibrium Limit, Phys. Rev. D80, 085011 (2009), 0904.3600 .

[63] B. Garbrecht and M. Garny, Finite Width in out-of-Equilibrium Propagators and Kinetic Theory (2011), 1108.3688.

[64] M. Plumacher, Baryon asymmetry, neutrino mixing and supersymmetric SO(10) unification (1998), hep-ph/9807557.

[65] L. Fonda, G. Ghirardi and A. Rimini, Decay Theory of Unstable Quantum Systems, Rept.Prog.Phys. 41, 587-631 (1978).

[66] S. Blanchet, T. Hambye and F.-X. Josse-Michaux, Reconciling leptogenesis with observable mu $\rightarrow$ e gamma rates, JHEP 04, 023 (2010), 0912.3153.

[67] B. Garbrecht and M. Herranen, Effective Theory of Resonant Leptogenesis in the Closed-Time-Path Approach (2011), 1112.5954.

[68] S. Davidson, E. Nardi and Y. Nir, Leptogenesis, Phys.Rept. 466, 105-177 (2008), 0802.2962.

[69] W. Buchmuller, P. Di Bari and M. Plumacher, Leptogenesis for pedestrians, Annals Phys. 315, 305-351 (2005), hep-ph/0401240. 